\title{
Protein sequences
}

\section{VERTEBRATES}

\section{PTPRQ}

>PTPRQ.human gi|222537743|ref|NP_001138498.1 | phosphatidylinositol phosphatase PTPRQ
precursor [Homo sapiens]
MDFLIIFLLLFIGTSETQVDVSNVVPGTRYDITISSISTTYTSPVTRIVTTNVTKPGPPVFLAGERVGSAGILLSWNTPPNPNG
RIISYIVKYKEVCPWMQTVYTQVRSKPDSLEVLLTNLNPGTTYEIKVAAENSAGIGVFSDPFLFQTAESA(15) PGKVVNLTVE
AYNASAVKLIWYLPRQPNGKITSFKISVKHARSGIVVKDVSIRVEDILTGKLPECNENSESFLWSTASPSPTLGRVTPPSTTH
SSSTLTQNEISSVWKEPISFVVTHLRPYTTYLFEVSAATEAGYIDSTIVRTPESV(14) PEGPPQNCVTGNITGKSFSILWDP
PTIVTGKFSYRVELYGPSGRILDNSTKDLKEATNLTPFTMYDVYIAAETSAGTGPKSNISVETPPDV(13) PGAVFDLLAEV ESTQVRITWKKPRQPNG I INQYRVKVLVPETG I I LENTLLTGNNEY INDPMAPE IVN IVEPMVGLYEGSAEMSS DLHSLATF IY NSHPDKNFPARNRAEDQTSPVVTTRNQYITDIAAEQLSYVIRRLVPFTEHMISVSAFT IMGEGPPTVLSVRTRQQV

E (12) PESSPQDVEVI DVTADE IRLKWS P PEKPNGI I I AYEVLYKN IDTLYMKNTSTTDI I LRNLRPHTLYNI SVRSYTRE GHGNQVSSLLSVRTSETV(11)

KKYTQYIIEVSASTLKGEGVRSAP IS ILTEEDA (10) PDS P PQDESVKQLSGVTVKLSWQP PLE PNGI I LYYTVYVWNRSS LKT YSVYYRNTSGTFMQNFTLHEVTNDFDNMTVSTI IDKLTIFSYYTFWLTASTSVGNGNKS S D I EVYTDQDI (8) PEGFVGNLTY ES ISSTAINVSWVPPAQPNGLVFYYVSLILQQTPRHVRPPLVTYERS IYFDNLEKYTDYILKITPSTEKGFSDTYTAQLYIKTE EDV (7) PETSPI INTFKNLSSTSVLLSWDPPVKPNGA I ISYDLTLQGPNENYSFITS DNYI ILEELSPFTLYSFFAAARTRKGI GPSS I LFFYTDESV (6) PLAP PQNLTL INCTSDFVWLKWS PSPLPGG IVKVYSFKI HEHETDT IYYKN I SGFKTEAKLVGLEPV STYS IRVSAFTKVGNGNQFSNVVKFTTQESV (5) PDVVQNMQCMATSWQSVLVKWDP PKKANGI ITQYMVTVERNSTKVSPQDH MYTF IKLLANTSYVFKVRASTSAGEGDESTCHVSTLPETV (4) PSVPTNIAF SDVQSTSATLTWIRPDT I LGYFQNYKITTQLR AQKCKEWESEECVEYQKIQYLYEAHLTEETVYGLKKFRWYRFQVAASTNAGYGNASNWISTKTLPGP (3) PDGPPENVHVVATS PFSISISWSEPAVITGPTCYLIDVKSVDNDEFNISFIKSNEENKTIEIKDLEIFTRYSVVITAFTGNISAAYVEGKSSAEMIVT TLESA (2) PKDP PNNMTFQKI PDEVTKFQLTFLPPSQPNGN IQVYQALVYREDDPTAVQ I HNLS I IQKTNT FVIAMLEGLKGGH TYNISVYAVNSAGAGPKVPMRITMDIKA (1) PARPKTKPTP IYDATGKLLVTSTTITIRMP ICYYSDDHGPI KNVQVLVTETGA QHDGNVTKWYDAYFNKARPYFTNEGFPNP PCTEGKTKF SGNEEIY I IGADNACMIPGNEDKICNGPLKPKKQYLFKFRATNIMG QFTDSDYSDPVKTLGEGLSERTVEI I LSVTLCILSI I LLGTAIFAFARIRQKQKEGGTYS PQDAEI IDTKLKLDQLITVADLEI KDERLTRLLSYRKS I KP I S KKSFLQHVEELCTNNNLKFQEEFSELPKFLQDLSSTDADLPWNRAKNRFPNIKPYNNNRVKLIAD ASVPGSDYINASYISGYLCPNEF IATQGPLPGTVGDFWRMVWETRAKTLVMLTQCFEKGRIRCHQYWPEDNKPVTVFGDIVITK LMEDVQIDWTIRDLKIERHGDCMTVRQCNFTAWPEHGVPENSAPLIHFVKLVRASRAHDTTPMIVHCSAGVGRTGVFIALDHLT QHINDHDFVDIYGLVAELRSERMCMVQNLAQYIFLHQCILDLLSNKGSNQP I CFVNYSALQKMD SLDAMEGDVE LEWEETTM

>PTPRQ.mouse gi|124487427|ref|NP 001074901.1| ENSMUST00000050702

phosphatidylinositol phosphatase PTPRQ precursor [Mus musculus]

MDFLFFFLFSLIGTSESOVDVSGSFDDTVYDITLSS ISATTYSS PVSRTLATNVSKPGPPVFLAGERVGSAGILLSWNTPPNPN GRI I SYVVKYKEVCPWMQTAYTRVRAKPDS LEVLLTNLNPGTTYEIKVAAENSAGI GVFS DP FLFQTAESA (15) PGKVVNLTV EALNYSAVNLIWYLPRQPNGKITSFKISVKHARSGIVVKDVS IKVEDLLSGKLPECNENSDSFLWSTTSPSPTLSRATPPLRTT HLSNTLARNKISSVWKEPISFVVTHLRPYTTYLFEVSAVTTEAGYIDSTIVRTPESV (14)

(13) PGAVFDLQIVE VEATEIRVSWRKPRQPNGI ISQYRVKVSVLESGVILENTLLTGQDEYINNPMTPEIMNLVDPMI GFYEGSGEMSSDLHSLASFI YNSHPHDFPARTRVEDQRSPVVATRNQYMTDIAAEHLSYVIRRLVPFTEHTISVSAFTVMGEGPPTVLTVRTREQVP

(12) PESSPQDVKVTDVSPSELSLTWSPPEKPNGI I AYEVFYQNADALFVKNTSTTNITLSDLKPYTLYNIS IQSYTRI GHGNQSSSLLSVRTSETV(11)

KKYTHYVIEVSASTLKGEGVRSMP IS ILTEEDA (10) PDSP PQNFSVKQLSGVTVMLSWQPPLEPNGI ILYYTVYVWDKVSLKT
INATEVSLELSDLDYHADYSAYVTASTRFGDGKTRSSVINFRTPEGE (9) PSDPPKDVHYVNLSSSSI LFWTP PVKPNGI IQY
YSVYYQNTS STFVQNFTLLEVTQEP GNVTVSARIYKLAVFSYYTFWLTASTLVGNGNKS SDVI HVYTDQDI (8) PEGGVGNLTY ESLSSTAINVSWTPPSQPNGLVFYYVSLNLQQS P PRHRRP PLTTYENS IYFDNLEKYTDY IFKITPSTEKGFSETYTAQLHIKT EEDV (7) PDTPP I INTFKNLSST I LLSWDP P LKPNGA I LSYHLTLQGTHANRT FVTS GNH IVLEELSPFTLYS FLAAARTMKG LGPSS I LFFYTDESA (6) PLAPPQNLTLINYTSDFVWLTWSPSPLPGGIVKVYSFK I HEHETDTVFYKNISGFQTDAKLAGLEP VSTYS I SVSAFTKVGNGNQF SNVVKFTTQESV (5) PDAVQNIACVARDWQSVSVMWDP PRKANGI I I HYMITVEGNSTKVSPRD PMYTFTKLLANTSYIFEVRASTSAGEGNESQCNVSTLPETV (4) PSVPTNTAFSNVQSTSVTLRWIKPDTILGYFQNYKITTQL RAQKCREWEPEECVEHQEVQYLYEANQTEDTVRGLKKFQWYRFQVAASTNAGYGNASSW ISTQTLPGP (3) PDGP PENVRVVAT SPFGINISWNEPAI ITGPTFYLIDVKSVDNDNFNIS FVKSNEENKTTEINDLEVETRYSVVITAFVGNVSGAYTDGKS SAEVI I TTLESV (2) PKDP PNNMTFQK IPDEVTKFQLSFLPPSQPNGNIQVYQALVYREDDPTAVQ I HNLS I IQKTDTSVIAMLEGLKGG HTYNISVYAINSAGA GPKVQMRI TMDIKA (1) PARPKTKP I P I HDATGKLLVTSTT IT IRMP ICYYNDDHGP IRNVQVLVAEAG 
AQQDGNVTKWYDAYFNKARPYFTNEGFPNPPCIEGKTKFSGNEEIYVIGADNACMI PGNEEKICNGPLKPKKQYLFKFRATNVM GQFTDSEYSDPIKTLGEGLSERTVEI I LSVTLC I LS I I LLGTAI FAFAR IRQKQKEGGTYS PRDAE I I DTKFKLDQLITVADLE LKDERLTRLLSYRKS I KPVSKKSFLQHVEELCTNNNLKFQEEFSELPKFLQDLSSTDADL PWNRAKNRFPNIKPYNNNRVKLIA DVSI PGSDYINASYVSGYLCPNEFIATQGPLPGTVGDFWRMVWETRAKTLVMLTQCFEKGRIRCHQYWPEDNKPVTVFGDILIT KLMEDIOIDWTIRDLKIERHGDCMTVROCNFTGWPEHGVPENTT PLIHFVKLVRTSRAHDATPMVVHCSAGVGRTGVF IALDHL TQHIHDHDFVDIYGLVAELRSERMCMVQNLAQY IFLHQCILDLLSNKGGHQPVCFVNYSTLQKMDS LDAMEGDVELEWEETTM

PPTPRQ.chicken XP_001235338.2। PREDICTED: phosphotidylinositol phosphatase PTPRQ ENSGALT00000017794[Gallus gallus]

MPDTGVFDGIYVTTNGGPNATFSLKSDGKLTVENLTPGTEYDFCVFTKSREMLSSSYRVTGVKTCLAAPLNIREGNVTDTSVQI AWDRAEGDFQQYEVTCTNCASAFRVQKVKQETATFSNLVPGKLYSFTVRTEKEGFRDSVLVAKEIETV(20)

C (19) PDP P S

LQVLGQEENTVYLSWKLPRGGFDKFQLSYCLMNNEKPFTRTVYDSRTVVKNLAPGMEYTFQLRTIKGWDSSVAVEKNVITK (18 ) PAGICNLALKMVNTSSATLMWNPTKTNFTSYKASLSNTTF ISKFMI PGAVSKFSVTNLTAGG IYNFTLQRLQGNIEGSPAFLE IVAE (17) PAKPEGLKF FNVS SN SF SLYWRLPYGHVDRFCVDL I PDHGSVVI SDLGVREYQADFYNTTPGTTYNVTVSTVSS ST YSSPASRTVTTNVTN (16) PGPPAFLAGERVGSAGI LLSWNTPQHPNGR I LSY IVKYKEVCPWMQTAYTQVTSKPDSLEVLLTN LNPGTTYE IKVAAENSAGVGVFSAPFLFQTAESA (15) PGKVVNLTVEALNYSAVNL IWF LPRQPNGK ITSEKI SVKHARSGIV VKDVLVKVEDLLSGRLPECNDNSESFLWSTTTPSTTFGKSTIPSSTTVTASTEASSQMSAVWNEPISFVITNLRPYTTYLFEVS AVNTEAGYIDSTIVRTPESV (14)

(13) PGAVSDLHLVEVEATYIKIVWRKPQQPNGIITQYRVKVHVQEKEVTLE NI ILIGKNKHLIDSLEPYMINENIEPSPTWNS IETANELYEGSGEMFS S IQTVSPVILTTVSDDHLPA INGAAELHSTLDDQYA TDILGEELSYIIKGLVPFTDYTISVSAFTAIGEGPPSVLTVRTREQV WLPPEQPNGLITHYEVLYSDSS DLFVRNTSSTNI SLTEMMPYTLYNISVRAFTRLGHGNQSSEPLLVRTSETV(11)

(12) PDSPPONVEI INVTATEINLK

\section{A (10) PSSP PESLSVKQLSGVTVKLSWKPPLEPNGI I LYYTVYV}

A (9) PGDP PKDIVYKNLTSTS IML FWS P POKPNGNIRYYSVYFRNDSGIFIONFT S HDNDS DVNMSP SAVLDDLAKYSHYTLWLTASTAVGDGNKTSEI I DVYTDQDI (8) PDGPVENLVYQNI SSSSVNVSWLPPSQPNGLVFFHVS LSL LQLGTNKI LSFLTYNTS I IFDNLEKYTDY I LKITPATDKGSSELHALSLHIRTDEDV (7) PESAPI IKTFSNLSTTSVMLSWDP PVKPSGII I YYDLNLFGSEKNISFSTTNNFI I LEDLVPSTLYS IYTAARTMKGSGPSSVLHFYTDESV (6) PLAPPQNLTITNY TADSVWLKWEPSPQPNGVITRYNLKIYQNDTEKIFYQNISGSNNEAKLDGLKPFSTYFISVSAFTKLGNGNQFSNAVQFTTMES

$V$ (5) PDVVQNVHCIATSWES I FMQWEPPASSNGVITYYIVTVEGS STNFSSDTTLHTFQNLLSNISYQFKIKAAT SAGDGEEQI CNASTLPEEV (4) PSAPRD IVFSNVQSTSVTLNWRS PKS I PGYFQNYKITTQLQS I YCSNWETKECIEDEI HQYLYEKGVNAQI EETVYGLKKYRWYRFAVAASTNAGYGSSSPWISTQTLPGS (3) PDGPPENVTVLATSPHS INISWSEPVVITGPTCYLIDITSV DNENYKAQFLRTNDEGKILEISDLKAFTRYSVVI IAFTGDVSAAAIEGKASSPVIVSTFEAV (2) PEDP PNNVTFQKIPDEVTK FQVTFVPPSE PNGNIQVYQAMVYNEDDPAAIRI HNLSVIDKTDQSVTAMIEGLKGGHTYNVSVYAINGAGAGPKIQLKITMDIK E (1) PPRPKKKPAPVYDTNGALLVTATT ITIRMPVCYYSDDHGP I KK IQVLVVEAGAQHDGNVTKWHDAYFNRPRPYFTNEGEP NPPCIEGKEDLSGKEEIYVIGADTTCMISGSQDKICNGPLKPRKQYLFKFRATNVKGQFTDSDYSDPVKTLGEGRSGGSVEV AVTLC I LSVVLLVAAVYAFARIRQKQKEGGTYS PRDAEI IDTKFKLDQLITVADLELKDERFTRYSSFFFRRKEIFVIQLLSYR KS IKP I KKSFLOHVEELCTNNNLKFQEEFSELPKFLEDLASTDADLPWNRSKNRFPNIKPYNNNRVKLMPDAGIPGSDYINAS YVSGYLCPNEFIATQGPLPGTVGDFWRMVWETRAKTLVMLTQCFEKGRIRCHQYWPEDNKPVTVFGDIVITKLVEDIQIDWTIR DLKIERHGDCMMVRQCNFTSWPEHGVPETTAPI IHFVKL IRASRAHDNT PMVVHCSAGVGRTGVY IALDHLTQH INDHDFVDIY GLVAELRSERMCMVQNLAQY IFLHQCVLDLLTSRGS SQP ICFVNY SALQKMD S LDAMEGDVELEWEETTM

PPTPRQ.fish IXP 009291679.PREDICTED: phosphotidylinositol phosphatase PTPRQ ptprq001 A8DZA4 ENSDART00000143740[Danio rerio]

MTTTAAAQDTFRYGLTDHHPLQHQLDLHESSVS IHDLTPGSDYSFDIQSFLGSDFSQTTSKNISTRPAGVCSLFVSDVNVTSAT VSWAAAAGGFDFYRVIVRNS SHKWT I DAS PHLQEVTVSGLWSGCSYNTTVQRFRNT I S GAAATINI HSV (17) PAS PEGLRVIS VSPRSFSLHWLAS PGCEKTYQVQLY PDHGNINITTDTADNNVQAQVSSVTPGTSYTVTVNAVAS SGFS SPVSRILVTTIESS (1 6) APSKPEGERVGSTGILLSWRMPMP LDPS IHSFVIRYKEMCPYPDPSFTEITKSLDIPETLLNTLTPGATYNIKVAAVNKAGV GPFSQSLYYKTAEAP (15) PGLVSNLTAFAEDHTSVIVTWFLPVRINGLITKFAVKVKHARTGQIVRTKELNAEDIMNGALPHC NDAADILSRGTP S PSQTSQLTSAVLPPITLSAVPPAS IWSVPISVKI DE LRPYTPYVFEVSAFT SDGEGQIASTMVRMPEAA (1 4)

A(13) PSAVSLLKAEAVDSTSVRLSWRSPIQPNGLITHYRILVLYHDTLVQDITLRGQNLISPLRRNARSLDFTT ESVFTLTSHETFSTLGRDFTNTDTEAPSALPLTPSTPHTPPPWLTVTHDRTDASVQTQTDAVTEDLTTEHPPVTPVLPATEVSA DLSSDQITHVVRRLSPFTEYKFSVSAKNIIGEGPSTEVTVKTNEQV

V(12) PDSPPRGLTIVKTTSSTATLSW SPPEKPNG I IRMYEISYTNGTYSNTVNSTSASATLRYLKPHTHYNVTVRAFTLLGHGEQ I SETLQMLSGEDV (11) PGSPPYDI SYEST GSS SVNVSWSPPLIANGVI IFYNVEYWNATOSLNOTTHMPYIVLSNLRKYAHYRI SVOAATOVGMGNHTSEILNITTLE DV (10) PSSPPKELIARKLSDTEVELSWEAPEEANSE ILYY IVRV

GLLIYITESTIESA (9) PSDP PQDVVYTLLT ISTVRLSWRP PNEPNGI IQYYT IYYTDNNTEYTERVPGSEHQLLLSDLQAGQD YSVWMSSSTSVGDGGLFS PSLNETTLEDV (8) PSGPVHNLSATIYSSTAVVISWDP PLEPNGRVYYQLSLQEAGITHPSINRTV 
NMTITKTTTDTIYLFTKLRKYFPYIINVTPATSAGSAVNHTTMLHLRTDEDV (7) PSSPPLSGSNKNLSSTS IQVSWFPPVEAN GEIIEYAVNLQGPSTSNKNYTSETHLVLSELTPFTPYNLS IAAVTRVGTGP PVVLSLHTDEAG (6) PMS PPRNLTIFNHTANSV WLQWEPSPEPNGVVQLYGFRI LELNTDSFRYQNSSDASTQAELGGFKPHNSYEISVCTFTRAGNGDQYSLPVLFTTNESV (5)S DAVGNLSCSGLDWDSVYMEWELPDHPNGE I LYYL IRSGDLEDEAH PVVLTHTVAHTLTGLS PHAFYLITVAAVNSAGVGEEANC SAHTP PESV (4) PGP SHSLTVTDVT SDSVSVSWQRPVHVPGLLQGYNVE IEQLSRNCERQQQQVESCMEAQ I LEWVEGETTRAT LTSLLKYRQYRIRVVAFTRAGAGEPSEWIYTQTLAGN (3) PDAPPGAISVVPSANGLKIEWDKPSVISGPTSYIIDITALDGSG YNITLVRHSEEIRTVIVGNLSAFTLHSVTITAFTGPLSNARRDGKASEPVLIRTLEDE (2) PKDPPKNVTLTVIPEEVTRVYVT FSPPDE PNGNISAYRVDI YRNGQLDFFINSLSVISNPNNTMTA I I DGLKGGFNYS IRIAAVNGAPGFGPSSEVHVTTGVKA (1) PPKPKKTPRAALNSAGVI ISTSKTITIEMPECFFTDDHGPIQKVQVIVSEPAVMDYGNLSNWKSVFLHPTAPYLTDDGFLNPEC PKNSERMS S STKTYVI GEDEGCLSEDAETLCNGPLKPKTHYVFKFRATNIRGQFTDSEYSDKVRTADDRLLTRDEQI ILGVLLS FFLALFLIL I IYGSVK I HRRKKEGGTYS PRQAE I IETKFKLDQL I AVADLE LKEEK I NRL LS YRKS LKP I SKKSFLQHVEDLCA NDNAKFQEEFAELPKLLQDLATSDADLPWNRSKNRFTNIKPYNNSRVKLLSEPGMPGSDYINASFVSGYLCPNEFIATQGPLPS TVADFWRMIWETGTKTIVMLTQCFEKGRIRCHQYWPEDNKPVTVFADI I ITKLTEDVRPDWTVRALKVERHGSYMIVHHFNYTS WPEHGVPESSSTLVQFVKAVRSNRGHENTT IVVHCSAGVGRTGVF IALDHLIQHLRDHEFVDIYGLVAELRSERMCMVQNLAQY MFLHQSTLDLLSAKGNSQS IWFVNYSALEKMDSLDAMEGDVELEWEETTM

\section{PTPRB}

>PTPRB.human PTPRB-001 ENST000003344142200 [Homo sapiens]

MEAEFYMVILTCLIFRNSEGFQIVHVQKQQCLFKNEKVVVGSCNRTIQNQQWMWTEDEKLLHVKSALCLAISNSSRGPSRSAIL DRCSQAPRWTCYDQEGF LEVENAS LF LQKQGSRVVVKKARKYLHSWMKI DVNKEGKLVNES LCLQKAGLGAEVSVRSTRNTAPP QILTTFNAVPDGLVFLIRNTTEAF IRNAAENYSQNSSERQHPNLHMTGITDTSWVLSTTQPFSSTTEETGLAEPERCNFTLAES KASSHSVS IQWRI LGS PCNFS L IYS SDTLGAALCPTFRIDNTTYGCNLQDLQAGTIYNFR I I SLDEERTVVLQTDPLPPARFGV SKEKTTSTSLHVWWTPSSGKVTSYEVQLFDENNQKIQGVQIQESTSWNEYTFFNLTAGSKYNIAITAVSGGKRSFSVYTNGSTV (15) P SPVKDI GI STKANS LLISWS HGS GNVERYRLMLMDKGI LVHGGVVDKHATSYAFHGLTPGYLYNLTVMTEAAGLQNYRW KLVRTA (14) PMEVSNLKVTNDGS LTS LKVKWQRP P GNVDS YN I T LS HKGT I KESRVLAPWITETHFKE LVP GRLYQVTVSCVS GELSAQKMAVGRTF (13) PDKVANLEANNNGRMRSLVVSWSPPAGDWEQYRI LLFNDSVVLLNITVGKEETQYVMDDTGLVPGR QYEVEVIVES GNLKNSERCQGRTV (12) PLAVLQLRVKHANETS LS IMWQTPVAEWEKY I I L LADRDLLL IHKS LSKDAKEFTF TDLVPGRKYMATVTSISGDLKNSSSVKGRTV(11) PAQVTDLHVANQGMTSS LFTNWTQAQGDVEFYQVLLI HENVVIKNES IS SETSRYSFHSLKSGSLYSVVVTTVSGGISSRQVVVEGRTV(10) PSSVSGVTVNNSGRNDYLSVSWLLAPGDVDNYEVTLSHDG KVVQSLVIAKSVRECSFSSLTPGRLYTVTITTRSGKYENHSFSQERTV (9) PDKVQGVSVSNSARSDYLRVSWVHATGDFDHYE VTIKNKNNF IQTKS I PKSENECVFVQLVPGRLYSVTVTTKSGQYEANEQGNGRT I (8) PEPVKDLTLRNRSTEDLHVTWSGANG DVDQYEIQLLFNDMKVFP PFHLVNTATEYRETSLTPGRQYKILVLTISGDVQQSAFIEGFTV(7) PSAVKNIHISPNGATDSLT VNWT PGGGDVDSYTVSAFRHSQKVDSQTI PKHVFEHTFHRLEAGEQYQIMIASVSGS LKNQINVVGRTV (6) PASVQGVIADNA YSSYSLIVSWQKAAGVAERYDI LLLTENG I LLRNTSEPATTKQHKFEDLTPGKKYKIQI LTVSGGLFSKEAQTEGRTV (5) PAA VTDLRITENSTRHLSFRWTASEGELSWYNI FLYNPDGNLQERAQVDPLVQSFSFQNLLQGRMYKMVIVTHSGELSNESFIFGRT V(4) PASVSHLRGSNRNTTDSLWFNWSPASGDFDFYELILYNPNGTKKENWKDKDLTEWRFQGLVPGRKYVLWVVTHSGDLSNK VTAESRTA (3) PSPPS LMSFADIANTS LAITWKGP PDWTDYNDFE LQWLPRDALTVFNPYNNRKSEGRIVYGLRPGRSYQFNVK TVSGDSWKTYSKPIFGSVRTK (2) PDKIQNLHCRPQNSTAIACSWI PPDSDF DGYS IECRKMDTQEVEFSRKLEKEKS LLNIMM LVPHKRYLVS IKVQSAGMTSEVVEDSTITMIDR (1) PP PP P PHIRVNEKDVLISKS S INFTVNCSWFS DTNGAVKYFTVVVREA DGSDELKPEQQHPLPSYLEYRHNAS IRVYQTNYFASKCAENPNSNSKSFNIKLGAEMESLGGKCDPTQQKFCDGPLKPHTAYRI SIRAFTQLFDEDLKEFTKPLYSDTFFSLPITTESEPLFGA IEGVSAGLF L IGMLVAVVALL ICRQKVS HGRERPSARLS IRRDR PLSVHLNLGQKGNRKTSCP I K INQFEGHFMKLQADSNYLLSKEYEELKDVGRNQSCDIALLPENRGKNRYNNILPYDATRVKLS NVDDD PCSDYINASYI PGNNFRREYIVTQGPLPGTKDDFWKMVWEQNVHNIVMVTQCVEKGRVKCDHYWPADQDSLYYGDLILQ MLSESVLPEWTIREFKICGEEQLDAHRLIRHFHYTVWPDHGVPETTQSLIQFVRTVRDYINRS PGAGPTVVHCSAGVGRTGTFI ALDRILQQLDSKDSVDIYGAVHDLRLHRVHMVQTECQYVYLHQCVRDVLRARKLRSEQENP LFP IYENVNPEYHRDPVYSRH

\section{>PTPRB.mouse 201 ENSMUST00000092167 [Mus musculus]}

MLRHGALTALWITLSVVQTGVAEQVKCNFTLLESRVSSLSAS IQWRTFASPCNFSLIYSSDTSGPMWCHPIRIDNFTYGCNPKD LQAGTVYNFRIVSLDGEESTLVLQTDPLPPARFEVNREKTASTTLQVRWTPSSGKVSWYEVQLFDHNNQKIQEVQVQESTTWSQ YTFLNLTEGNSYKVAITAVSGEKRSFPVYINGSTV(15) PSPVKDLGISPNPNSLLISWSRGSGNVEQYRLVLMDKGAIVQDTN VDRRDTSYAFHELTPGHLYNLTIVTMASGLQNSRWKLVRTA (14) PMEVSNLKVTNDGRLTSLNVKWQKPPGDVDSYS ITLSHQ GTIKESKTLAPPVTETQFKDLVPGRLYQVTISCISGELSAEKSAAGRTV(13) PEKVRNLVSYNEIWMKSETVNWTPPAGDWEH YRIVLFNESLVLLNTTVGKEETHYALDGLELIPGRQYEIEVIVESGNLRNSERCQGRTV(12) PLAVLQLRVKHANETSLGITW RAPLGEWEKYI ISLMDRELLVIHKSLSKDAKEFTFTDLMPGRNYKATVTSMSGDLKQSSS IKGRTV (11) PAQVTDLHVNNQGM TSSLFTNWTKALGDVEFYQVLLIHENVVVKNESVSSDTSRYSFRALKPGSLYSVVVTTVSGGISSRQVVAEGRTV (10) PSSVS GVTVNNSGRNDYLSVSWLPAPGEVDHYVVSLSHEGKVDQFLIIAKSVSECSFSSLTPGRLYNVTVTTKSGNYASHSFTEERTV( 9) PDKVQGISVSNSARS DYLKVSWVHATGDFDHYEVTIKNRESFIQTKTIPKSENECEFIELVPGRLYSVTVSTKSGQYEASEQ GTGRTI (8) PEPVKDLTLLNRSTEDLHVTWSRANGDVDQYEVQLLFNDMKVFPHIHLVNTATEYKFTALTPGRHYKILVLTISG 
DVQQSAFIEGLTV (7) PSTVKNIHISANGATDRLMVTWSPGGGDVDSYVVSAFRQDEKVDSQTIPKHASEHTFHRLEAGAKYRI AIVSVSGSLRNQ I DALGQTV (6) PASVQGVVAANAYSSNSLTVSWQKALGVAERYDI LLLNENGLLLSNVSEPATARQHKFEDL TPGKKYKMQ ILTVSGGLFSKESQAEGRTV (5) PAAVTNLRITENSSRYLSFGWTASEGELSWYNIFLYNPDRTLQERAQVDPLV QSFSFQNLLQGRMYKMVIVTHS GELSNESFIFGRTV (4) PAAVNHLKGS HRNTTDSLWFSWS PASGDFDFYELILYNPNGTKKE NWKEKDVTEWRFQGLVPGRKYTLYVVTHSGDLSNKVTGEGRTA (3) PSPPSLLSFADVANTSLAITWKGPPDWTDYNDFELQWF PGDALTIFNPYS SRKSEGRIVYGLHPGRSYQFSVKTVSGDSWKTYSKPISGSVRTK (2) PDKIQNLHCRPQNSTAIACSWIPPD SDFDGYSIECRKMDTQE IEFSRKLEKEKSLLNIMMLVPHKRYLVS IKVQSAGMTSEVVEDSTITMIDR(1) PPQPPPHIRVNEK DVLISKSS INFTVNCSWFSDTNGAVKYFAVVVREADSMDELKPEQQHPLPSYLEYRHNAS IRVYQTNYFASKCAESPDSSSKSF NIKLGAEMDS LGGKCDPSQQKFCDGPLKPHTAYRIS IRAFTQLFDEDLKEFTKPLYSDTFFSMPITTESEPLFGVIEG

ICRQKASHSRERPSARLS IRRDRPLSVHLNLGQKGNRKTSCP IK INQFEGHFMKLQADSNYLLSKEYEDLKD VGRSQSCDIALLPENRGKNRYNNILPYDASRVKLCNVDDD PCSDY INASY IPGNNFRREYIATQGPLPGTKDDFWKMAWEQNVH NIVMVTQCVEKGRVKCDHYWPADQDPLYYGDLILQMVSESVLPEWTIREFKICSEEQLDAHRLIRHFHYTVWPDHGVPETTQSI IQFVRTVRDYINRSPGAGPTVVHCSAGVGRTGTFVALDRILQQLDSKDSVDIYGAVHDLRLHRVHMVQTECQYVYLHQCVRDVL RAKKLRNEQENPLFPIYENVNPEYHRDAIYSRH

>PTPRB.chicken PTPRB-201 ENSGALT00000016330 [Gallus gallus]

MSSPNQQWQWMADDKLFHMKSGQCLS ISTRSALSSRS I I INCSQAVRWTCHEEDGLLKVANSS LFLTKQGQKVMAKQSKKYLHT WMQLEASETGKPVYVNLCSKQAAHNS LS TGLPL PNF PDLWMGTMNS TTTTSS P LNTTT SRP DNL PASS STQMS IRNVTEHFTKN FIENLYFDLKSAVTEKPTVPTTPPQYSSTTEETKSAYLLTDRGKTHSCSF IYALFQS PNTHKQLWQRRGMQVPAPYTEETNSEH SVPPVFRFHVDKPQLSNLRANLASS LAKCLQSVSVKDCTTHLLKAHLFSTTLEI IMLTILYTYITTNITSKSALLRWSAAAHAC NFNLTGRSEDGRPAGCRPASAGNGSYGCTVRDLEAGTWYHLRIEPLADGEAANVTLQTDPLPPPRFEINKEKTTFTSLQVRWDP SPGKVDLYNIVVFDHSNKKLQESS I PGRFSKTEETFTGLVPGNKYNIVLTAVAGNKSTPEFRINGSTA (15) VPSPVKNIQVIV KTDS IHASWS PGS GHVDLYKLVLF DNHGTVHES HQENPLTSYIF SGLTAGHLYNLSVITQAAELESTAFRIVRTA (14) PAEVI DLTVTNDDSFDTLKVKWRRPSGGVDFYNITLSHLRSVKEVKTLQPAVTETHFDKLTPGRLYQITART ISGELFTDKMATGRTF ( 13) PQKVSELKA GGGGWLRTLRVNWLPPAGDLERYHLLLWNHSALVLNTTLGKNVTEYLVRDVGL I PGRQYEVEVVVESGDLQS RASCTGRTA (12) PEPVLQLRVKHANE S S LSVMWVT PVAEWDSYVVS LGDRDLTVIKKGLGKEAKEFTFNELVPGRKYTATVTT ISGI LSNWTSVEGRTV(11) PAQVTGLTVASQGSTNS LFTNWTKALGDVDSYQVLL I HENVVIKNETVPSETNEYHFYPLKPGG LYSVVVTTVSGGISSRQTIAEGRTV(10) PSSVTGVTVNNSGRSDYLSVSWLPASGDVDSYLVTLSHDDQIIQTLTISKS LSEC SFSSLTPGTLYNVMITTKSGKYENHSFSRERTV(9) PSGVQGLTVSNSARSDYLKVSWLHASGSFDSYEVIIKNNNDFIQTKSV PKDENECVFTNLVPGRQYSVTVSTRSGKYETSERVFGRTM(8) PESVKGLTLSNRSTEDLRVTWSKAEGDVDKYEIQLLYNDMK IFPP IFLGNT IEECWFTALTPGRLYKIVVLTISGDAQRATFIEGLTI (7) PSAVRN INVS PNGMTDSLKVSWTPGGGDVDSYTV TIFQQNHQLDSRSVSKDVSEHTFHDLEAGEQYRVVVQSNSGALHNSLAAFGRTI (6) PASVQELLADHAYSSHSLLVSWQKAPG VAERYDILLLNEQGVLLSNKSEPATAKQHKFEDLLPGKKYKIHILTVSGGLFSKRAETVGRTV(5) PAAVTNLKVTKNTTDQLS FSWTTSQGELDSYDIF L YNPDKSLHDRIS GEQHLQQCSFQNLRQGRMYRMVIVTHSGDLTNESSVFGRTA (4) PAPVVGLKASN RNMTDSLWFTWNPAAGDVDFYEFNLYNPNGTQKESLQGKDLKEWYFQGLVPGRKYTLVVVTHSGDLINTANAEGRTA (3) PSPP NTVSFTDVANTS LS ITWLGP PDWTDYDDFELQWLPKDPLTVFNPYSS SKSKVRI IYGLRPGRLYKFSVRTVSGDSWKTYSQSQS ESVRTR (2) PDKIQS LHCRPQTSTAIACSWTPPDSDFDGYSVECKKLDTREVEFSKRIEKDRTLLNIMTLVPHKRYLVS IKVHS ADMT SEVVEDSTITMIDR(1) PPQPPPDIRVNKKEVLITKSS INFTFNCSWFSDTNGAVKYFTVVVREADGSEGPKPDEQHPLP SYLEYKHNDS IRIYQTNYFASRCAENPDSDYKSFDIKLGGEMENLGGKCDPDQQKFCDGPLKPRTAYRIS IRAFTQLFSEDPKF LPQPLFADTFFS LPITTEAEPLFGVIEGVSAGLFLIVMLVAVTALFVCRQKVSNGHERPTARLS IRRDKPLAVHLNLGQKGNRK TSSP I KVSHFEAHFTKLQADSNYLLSKEYEDLKDVGRNQTCDIALLPENRGKNRYNNILPYDTSRVKLSNVDDDPCSDYINASY I PGNNFRREY IATQGPLPGTKDEFWKMAWEQNVHNIVMVTQCVEKGRVKCDHYWPLDQDSLYYGDLIVEMLSESVLPEWT IREF KICSEEQLDSTRLIRHFHYTVWPDHGVPETTQSLIQFVRTVRDYINRTPDTGPTVVHCSAGVGRTGTFIALDRILQQLDSKDTV DIYAAVHDLRLHRVHMVQTECQYVYLHQCVRDVLRARKLRSEQENPLFP IYENVNPEYHRDAVYSRH

>PTPRB.frog xenpopus ptprb-201 ENSXETT00000024858 Xenopus tropicalis

MLRYAFGLSLWIVLNLIKNVEPVKCTINNTDVTVTSQS IYLEWTS PGSHCNFSLTCSSHNFWEITCNP IQKSNDSYECTLSGLE AGTVFDLRIVSLQDGEMKNLSLQTDPLPP SNFVVNKNSVTSSSVQANWAPSSGKVDLYKVKLLDSKKEIVQEIEVSGISLTKEV TFKNLTPGTKYS IEITAISGNKRSPTSVINASTV(13) PSTVKS I DANSKTDTI DASWKPGHGN I DSYKLILLNEDRKITEVVL EQNITSYSFHGLLPGYVYNLTVISEAAGLRTWNSKLIRTA (12) PAEVQAISVQSDGRTDALKVQWKEPRGKLDFYNVTLSDTG SIKYSRTVHPGTPTEITFTGLTPGHLYQVGISTIAGELSTSRMATGQTI (11)PEKVADLKAVSNGLRSLRLSWVPPSGDWDKY NIVLYNNTAVLVNTTI DKKYREYVIQDIGLIPGRQYEAVVIVESGKFKNLARCKGRTA (10) PPAVMDLRLKHANESS LT IMWV MPVAEWDNYVVSLADRDLTPVNKVLSKEAKEFTFNHLAQGRKYIATVTS ISGDLSSRASVEGRTV(9) PAQVTNLNVENQGTTS SLHADWTKAPGDVDSYQVLLIHEKIVIKNETVSNEINKYSFHSLKPGGLYSVVVTTVSGG ISSRQTVSEGRTGRKLLSIISS (8 ) NTGSLTPFLHQSWSSNSLRYIGWTSLICQGKFLAKDLFFAWPGIECSFPCVIKGALYFVRMDYQQTVGTNRYPRDNEAIPLVV VNTCCINCLSSSYRKLSVIES ISP INKRNNIKKRSVLSVS SEYTHPRLRHSGCVTTRANSGMLKLQLPGAGLLVPEAVKKLTLA ERSSEELHVTWSRADGDVDHYE IQLLFNDMKVFPPITLNNTADEYKFTSLTPGRLYKIVVLTFSGEAQRAMFVEALTV (7) PST VKNI I I SPNGMTNS LKVNWTP GGGDVDSYTVS I FHHNSP I GSHTTSKH I FEHTFQNLNPGELYKVDVQTNSGS LHKS LESFGRT I (6) PATVHSLSADNAYSSHLLIVSWQSAKGVTDRYDILLLSDHGIVITNKSVPIMAKS HKFEDLVPGKKYRIQVFTVSGGLFS AAVETEGQTV (5) PAAVSDVKITGNSTDSLSFTWNSSEGELDSYGIFLYNPDNS LHDRKS GNPDLRHCSFQGLLPGRLYKMVIV THSGSLTNESS I HGRTV (4) PAPVS SLQVSNRNTSES LWFTWAPALGDVDI YELILYNPNGTEKEKIQDKDLTESHFQNLVPGR 
LYTLVIFTHSGDLFNKATAVGRTE (3)APQPPNSVSFADVKINSLS IMWLGP PDWTDYDDFELQWSPKDLLVVVNPYS IGRSKG RI INGLYPGRQYTFGVQSVSGNTGKTLSPAIFGTVRTK (2) P DKI HHLHCRPQTSTA I SC SWTP PDSDF DGYSVECRRNGSDDV EFSKRIEKDKSS ITISTLVPHKRYVVS IKVHSADMT SQVIEDSAITMIDR (1) P PPPPLLIRVNKKDTFISKSS IHFRFNCSWF SDTNGAVKYFTVIVSEADGNDNQRPEASLPLPSYADYKTNKSTKIYQTSYFP SRCAENPDYNIQSYKIKLGTGMELLGGKCDQN ENKYCDGPLSPRTSYRISVRAFTQLFTEEMRTFPEPLYSDTFFSLPITTEAGS LFFNKNITDLPLQIFPQTKAMILVVLCRYEQ DPMTTHLSSQIERI PSVHLNVGHI Q I GDR I SSRP ILTAQFEEHFSKLOTDSNYLLSREYENLKDFGRDQSSDTALLPENRGKNR YSNI LPYDSTRVKLANVDDD PCSDY INASYMPGINFRREY IATQGPLPATKDDFWKMVWEQNVHI IVMVTQCTERGRAKCDHYW PMDQDSYYYGDLIVQMLSESVLPEWTIREFKICSEDQIDAPRLVRHFHYTVWPDHGVPETTQSLIQFVRTVRDYINRTPGSGPT VVHCSAGVGRTGTFIVLDRMLQQVDTVDSVDIFGAVRDLRIHRMYMVQTECQYVYLYQCVRDVLRARKLRNEQDNPLFP I YEN $V$ NPEYHRDAVYLRH

\author{
>PTPRB.fish Zebrafish ptprb-202 ENSDART00000127299 NP_001303656.1 FN428722 Danio \\ rerio
}

MLRNKAFHAAVWAACGILSTMVVDATECS IEIVKVISSTES IRLTLDNANVKCQYTVSVKDRHTDSKGCRQDREHSKCQIESLD PGTWYHLDVISTLDEKQQSQRAVTLQTRPSAVENLQVSGDANSLDVSWQPGKGKTERYWIVLIDSSGRDSAWNSTVASTATSYT MKGL ISGRLYNITVVTEVGELQNSASTQAQTA (14) PASVSKLRTENNGDRNS I RVLWDKASGDVDSYLVSLTTPGSNS IEKVI PPDDTYVVFENLSPGKGYQVSVSTRSGALSNKTWITGKAD (13) PGKVSDLVIENLSVRGALKISWTPPS GEWEHIRVALSNGS EVLRNQTVGRTAKE ILLSGLNLLPGRVYRMAVSVENGGLANTVIYEGEIG (12) LPPVSQLHVRHSDETS LSALWAHAAS S S SR DGYI IQLFQSNT STVIQTRNLSRDMRECTFNVLTPGRLYDITVTTTAKI IRSSATLQGRTQ (11) PLKVNHLKLSNKGSTDSLN ASWEKPLGDLDFYHMLLLRDQQTVHNITASANTT SRLLPFLRPGALHK I LVTT ISGSQTSKLAEAECRTV (10) PAAVSDITVT NSGPDFLNVSWKAAEGDVDNYVVMLKDQEKIVHTLATSKATTECVFRSLVSGRLYS ISIATHSGSYRNQTLLQERTK (9)PSTV QNPTA I HSARDDF LKLYWNHASGDYDYYVVTIEHNGTRLQSQKLNRTQS DCAF SDLVPGRLYNLTVSTWSGQHYSAVSIYGRTF (8) PGAVGNLSLTERGGTFLRVSWT SAP GDVDHYELQ ILFNDTQVSAAVNLSSAIGEHLFSALTPGRLYKIVLSTHSGSYQRAE ILEGRTV(7) PSQVQSVHLSAGTADGS LRVSWSSADGDLDFYSVS LFQETHLQDSRRVPKH ITQAEFYNLI PGQLYSVTVQSVS GSQTNNSTTTGRTE (6) PSTVTGLRADNELSTHSLLVSWNPAVGVHDGYRLWLLDDGGTN I HNASVPAASRHHLFENLTPGRRY QAHVRTLSGTAESKDVVAEGQTR (5) PSAVSALHVCSNTSSDLSFCWASAVGWVDGYELYLYDRDETLHYHTTLGSDALGWSFT LLQPGTHYKMTITSKSGKLSNQSSVWAHTA (4) PASVPELHVENQGQTDS LLLSWTPAPGGLTGYSVTVDGREQRVGPEVTQVV FHSLVAGRLYLATVQTWSEDLSNSTTAVGRTV(3) PAAPSSVSVSCSGGSVDMKWHVPDTGDYEDFEVTWFPQDTLYISHLRPT QRILEGLHPGRLYNIS LRTVSGKRHSPVTYSRPVYHTIRTP (2) PLPAPS I HCFPLSSTSVSCSWTPPHSDYDGFVVQCHRQGS GKAVYTHTLGNHTLSQQFDRLEPFKNYTIYVAVMSGDKQSSTVKNSVITMIDR (1) PPVPPVTVRVNEHSAVITHFTIRFKFNC SWFSDANGAIRYFTIIATESNDVDNGLPEQRHPLPSYLDYRQNHS IKAYQTGYFHSTCAEGSDGKVQVFE INLGAGMKHLGGAC KLDPES IQHGSHLCDGPLRSRTSYRLSVRAFTQLFDEENREFPHPLYTDTYLSLPLLTQSAPRSGLTGG

YRKRAHK I AVQES PVMKMCKWKALPTS QMCLRIRSPVQAANFESHLAKLQSDSSYLLSEEFEGLKDVGRIQTQNAARLLG NRNKNRYNNILPYDSTRVRLSCLEDDPCSDY INANY I PGNNFRWEY IATQGPLPGTKDDFWRMVWEQNVHSLVMVTQCVERGMV KCDHYWPTDSEPLCYGDIVVQLLSEKVFPEWT IRDFKISCESQLRYPRMVRQFHYTIWPDHGVPDTTQSLIQFVRTVRDFINRT NSPGISVVHCSAGVGRTGTFIVLDRALQQLDRNCTVDIYGCVF'DLRLHRSYMVQTECQYAY IHQCVRDVLRARKLGCERDNPLF PIYNNII

\title{
PTPRJ
}

>PTPRJ.human ENSG00000149177 [Homo sapiens]

MKPAAREARLPPRS PGLRWALPLLLLLLRLGQ I LCAGGTPS P I PDPSVATVATGENGITQ IS STAESFHKQNGTGTPQVETNTS EDGESSGANDSLRTPEQGSNGTDGASQKTPSSTGPSPVFDIKAVS ISPTNVILTWKSNDTAASEYKYVVKHKMENEKTITVVHQ PWCNITGLRPATSYVFS ITPGI GNETWGDPRVIKVITE (8) P I PVS DLRVALTGVRKAALSWSNGNGTASCRVLLES I GS HEEI TQDSRLQVNISGLKPGVQYN INPYLLQSNKTKGDPLGTEGGLD (7) A.SNTERSRAGSPTAPVHDESLVGPVDP S SGQQSRDTEV LLVGLEPGTRYNATVYSQAANGTEGQPQAIEFRTN (6) AIQVFDVTAVNISATSLTLIWKVSDNES SSNYTYKIHVAGETDS SN LNVSEPRAVI PGLRSSTFYNITVCPVLGDIEGTPGFLQVHTP (5) PVPVSDFRVTVVSTTEIGLAWSSHDAESFQMHITQEGAG NSRVEITTNQSI I IGGLFPGTKYCEE IVPKGPNGTEGASRTVCNRTV(4) PSAVFDI HVVYVTTTEMWLDWKSPDGASEYVYHL VIESKHGSNHTSTYDKAITLQGLIPGTLYNITISPEVDHVWGDPNSTAQYTR (3) PSNVSNIDVSTNTTAATLSWQNFDDASPT YSYCLLIEKAGNSSNATQVVTDIGITDATVTELIPGSSYTVEIFAQVGDGIKS LEPGRKSFCTD (2) PASMASF DCEVVPKEPA LVLKWTCP PGANAGFELEVSSGAWNNATHLESCSSENGTEYRTEVTYLNFSTSYNIS ITTVSCGKMAAPTRNTCTTGITD (1) P PPPDGSPNITSVSHNSVKVKFSGFEASHGPIKAYAVILTTGEAGHPSADVLKYTYEDFKKGASDTYVTYLIRTEEKGRSQSLSE VLKYE I DVGNESTTLGYYNGKLEPLGSYRACVAGFTNITFHPQNKGLIDGAESYVSFSRYSDAVS LPQDPGV

FWRKKRKDAKNNEVSFSQIKPKKSKLIRVENFEAYFKKQQADSNCGFAEEYEDLKLVGISQPKYAAELAENRGK NRYNNVLPYDISRVKLSVQTHSTDDYINANYMPGYHSKKDFIATQGPLPNTLKDFWRMVWEKNVYAIIMLTKCVEQGRTKCEEY WPSKQAQDYGDITVAMT SEIVLPEWTIRDFTVKNIQTSESHPLRQFHFT SWPDHGVPDTTDLLINFRYLVRDYMKQSPPESPIL VHCSAGVGRTGTFIAIDRLIYQIENENTVDVYGIVYDLRMHRPLMVQTEDQYVFLNQCVLDIVRSOKDSKVDL IYQNTTAMT IY ENLAPVTTFGKTNGYIA 
>PTPRJ.mouse Ptprj-201 ENSMUST00000168621 [Mus musculus]

MTRGGGRGSSRGRGSRELGATRGGWAPLAPPREAPASLRPRPLRARRARLRRVAAAAAAAMSPGKPGAGGAGTRRTGWRRRRRR RRLETETRAPGFGHTAGRVPGTFQGAQGMKPAARETRTPPRSPGLRWALLPLLLLLRQGQVLCAGAAPNPIFDIEAVVSPTSVI LTWKHNDSGASECRIENKMESNLTFPVKNQTSCNITGLSPGTSYTFS I ISVTTNETLNKTITTE (8) PWPVSDLHVTSVGVTQA RLTWSNANGTASYRMLIEELTTHSSVNISGLKPGTNNSFAFPESNETQADFAVAEEVPD (7) ANGTKRIPVTNLSQLHKNSLVS VDPPSGQDPSLTEILLTDLKPDTQYNATIYSQAANGTEGQPRNKVFKTN (6) STQVSDVRAMNISASSMTLTWKSNYDGSRTSI VYKIHVAGGTHSVNQTVNKTEAI I LGLSSSTLYNITVHPFLGQTEGTPGFLQVYTS (5) PDQVSDFRVTNVSTRAIGLAWRSND SKSFEIFIKQDGGEKHRNASTGNQSYMVEDLKPGTSYHFEI IPRGPDGTEGLSSTVNGSTD (4) PSAVTDIRVVNISTTEMQLE WQNTDDASGYTYHLVLESKSGS I IRTNSSQKWITVGSLTPGTLYNVTIFPEVDQIQGISNSITQYTR(3)PSSVSHIEVNTTTT TAAIRWKNEDAASASYAYSVLILKTGDGSNVTSNFTKDPS I LIPELI PGVSYTVKILTQVGDGTTSLVPGWNLFCTE (2) PEPV TSFHCEVVPKEPALVLKWACPFGMYTGFELGVRSDSWDNMTRLENCT SDDDTECRTEVAYLNFSTSYNISIATLSCGKMALPAQ NICTTGITD (1) PPTPDGS PNITSVSHNSVKVKFSGEEASHGP IKAYAVILTTGEAAQPSADVLKYTYEDFKRGASDTYVTYLI RIEEKGQSQGLSEVLNYE IDVGNQSTTLGYYNGRLEPLGSYRACVAGFTNITYNLQNDGL INGDESYVSFSPYSEAVFLPQDE

WRKKRTDAKNNEVSFSQIKPKKSKL I RVENFEAYFKKQQADSNCGFAEEYEDLKLIGISI PKYTAE IAENRGKNRYNNVLPYDISRVKLSVQTHSTDDYINANYMPGYHSKKDFIATQGPLPNTLKDFWRMVWEKNVYAIVMLT KCVEQGRTKCEEYWPSKQAQDYGD ITVAMTSEVVLPEWT IRDFVVKNMQNSESHPLRQFHFT SWPDHGVPDTTDLLINFRYLVR DYMKQI PPESPILVHCSAGVGRTGTFIAIDRLIYQIENENTVDVYGIVYDLRMHRPLMVQTEDQYVFLNQCVLDIIRAQKDSKV DLIYQNTTAMT IYENLEPVSMFGKTNGYIA

>PTPRJ.chicken PTPRJ-202 ENSGALT00000045335 NP_989952.1I [Gallus gallus] MRRLPLLPPCPLLLLLLLPAEVRCTTACTDDCSLKNVTEEMGT S SNDELSSVNAT SGNRRLSEDVS LPGRAMS DQNSVAQPRAVL DLKTEYVGVTSVNLTWTVNDTASDSYTYRIEVRNGSS INNKTSDITDAEITGLIPGTLYTFTVFAVAADGQTAGEGASISLYTK PSPVLDLKAEYVGVTSVNLTWTVNDTASASYTYRIEVTSDSSIDSLTSSVTMAEITGLIPGTLYSFKVFAVAADNRTEADGAS I SLYTK (6) P SPVLDLKAEYVGVT SVNLTWTVNDTASASYTYRIEVTS DS S IDS LTSNVTMAEITGLIPGTLYNFTVEAVAADNR TEADGAFTSLYTK (5) PTPVTDLKAEHGVTSVS LNWMVNDTASDSYTYRIEVRNGHSVNNKTSNI PETEITGLNPGTLYTFTVF AVAADGETEGEGASISVYTK (4) PRAVLHLKTEYVGVTSVNLTWTVNDTDSASYTYRIEVRNGS S INNKTSDITDAEITGLDPG TLYIFTVFAVAADGQTAGEGAS IS LYTK (3) PSMVLNLKAEYVTMTSVNLTWMVNDAESASYTYRIEVAHESLINETMSNVTKS IVTYLIPGTSYNFTVFAIAADNQTEGEGAS ISQNTV (2)PSSVNAFQCEAVANMSYLTLKWNCPYGGYSGFDIEIFNGTWTKKO QSQFCGREGSEEIFKTEPLDYYKTYTVSVTTVSDGLTSLPVQKICKTSITD(1) PPVPNKAPLVKAVSHNSLSVEFPDFESVNG PLKAYAVMIVTEAEGCLPSKS DLDYTYNDFKOKMTATYVTYVIDVEEISSS SHSONGHNIVDVGKGNTMYGYENGPLIPLHSYR ASVAGFTNINFTVANK IMGEQSYVSFSPCSEVVS LPQDPGV IAGAVIGCLIAA I LAVVA IGGY I FWRRRKDKRNTEVSFS P IKS KMIKVENFESYFKKOQADSNCGFAEEYEELKSAGVHOPKFAAEIAENRGKNRYNNVLPYDISRVKLSNPSCTTDDYINANYMPG YSSKKAFIAAQGPLPNT IEDFWRMIWEKNI YS IVMLTKCVEQARTKCEQYWPDKQSKSYGDI IVTMVSEVVLPEWT IRDFNVEN ADTMESHTVRQFHFTSWPDHGVPETTDLLINFRHLVHEYSSONPIDS PILVHCSAGVGRTGTFIAIDRLIQQIEMENTVDVYGV VYDLRMHRPLMVQTEDQYVFLNQCVMDI IRSQKEKKT DLIYONTTAMA IYENFT PGPAFGKANGYHA

>PTPRJ.fish FN428723 NP_001129714.1I [Danio rerio]

MGQHYRLQAIVSRTTVLFVVLVSIH ḦGHCTDCSLCQYKTSTTTSE INVFVGNSSFCIAQNASFTNSSQTNITVTGLLPGNTYFL I ISCT SVCCENF SS SPAQVQN ITVVNYTNSSVTVAWTVPDGHVDSYTVNCSTQS I PN INDNNTT I SS LPPGCLYNLTVTS ISGV LTNVSNIVQFATK (12) PNPPEAVRVSGQTFSS ISLLWSTPLSMSGVAVSYGVSYVPVQPGVNLPLTQTTSSNMTNITNLLSGT QYTITVVTNGVNNLSSSF INLIGYTV (11) PKIVQNLAVSNVSTNSVSLTWLP PNGTSSLFSYNINISSLGQTFYTTSNSYQIT QLQPGTQYNCYVTTLIVAGNISGPSQFIQCNTK (10) PLPVSNLKA IPVGTTVILLSWTPSTALYLYNVSANGTWSTYNS SEGA NVTGLTPGNNYTFTVITVANGLSSEPVTISAFTG (9) LGKAI I ISA IGNTQTMTVQWTPPAGTVS LYSARILLMDGTVLNTKNQ TKTTSMMF SDLLPGTQYSVIVSS ISGPVKQDSDSVTNATL (8) PTPPGEISTTQSTNTLNISWAQPLNMSSVSYYFLLQYNNST LINCSQNYTSLTGLNAGF PYNI IMWTVGAMNYSSSSKSLTAFTN (7) P SVVTNVTVNEFTETSVTLSWQQNDPQQSGYSYLLNY KT INGTSNQTTVNNNTVKLEQLQSASQYNISI IALTPGGTKSDPQFITACSR (6) PNRVNNVSSVALNVSTVRLSWARPLQYNQ LVSYQVLVSNCTKNSMNMSTSTEMITVTNLQPGTLCQ I S IYSVACGLLGQPVNT SVLTM(5) PSTVQPVVNSQGSNNSLLVLWS HPDGGLDMY I LNISSDGWSDFSFLNSTENNYTFTQLKAATLYTVTLTTVKGSFQETSGAVVSATY (4) PNSPGMITVLFKNTHS VLLHWDIAQNMT PGSFNYSLSFWS SKNNSLYLT PNNTLLLDGLQSGT PYNVS LATVGPLNLQSESMRYNVTTM (3) PDPVKNLK VVSTTTNT I SVTWKMVDVPKYVVSVNNAVK IT S SNNMT I HNLQP S TQYNI SVQSSTFDNTEGEAVCLQDCTD (2) AAPVENVTC VGPNLTLPMLNLFWNHPLGGYMNFEVNLSSS ISEKTKNLNYNFTGLKYNKTYSVTLRTLGCGKSSTVKNISCTTGFTN (1) PVV PKITAASVSEQQYNKFALVIQSDAFNDSNGTVCSYGLLVSSGSFDCLDNNQNYNQCLLKTYDDWKAKQSDTFLAVVKTNVSKSE LETI I IGDGSKWNKYTNGELNAKGTYNFAIVAFTLLEVKDGKVDVSKSYYSVSAYQSVTLPENPV I

RKKRKEVSNSVP I HSLSDP IKVEDYEAYYKRQRADSFCGFAEEFEDLRPVGINQSKTVAVFPENKAKNRYNNVLPYD SSRVKLSVLSSPFDDY INANYMPGYITKKEFIAAQGPLPGTVNDFWRL IWEKNVHT IVMLTKCNEQGRVKCEEYWPAEMKTFSN LTVTTISDI PLEDWTLRDFEVKNMKTAEIRSVRHFHFTAWPDHGVPETTELLINFRHLVREHMDEYSRHSPTLVHCSAGVGRTG TFIAIDRLIFQIERDGVVDVYGI IHDLRMHRPLMVQTEDQYVFLNQCAMDIIKSRTGNNVDL IY NNTAALTIYENFEPLKKGKN GYHKA 


\title{
PTPRO
}

\begin{abstract}
>PTPRO.human ENSP00000281171 [Homo sapiens]
MGHLPTGIHGARRLLPLLWLFVLFKNATAFHVTVQDDNNIVVSLEASDVISPASVYVVKITGESKNYFFEFEEFNSTLPPPVIF KASYHGLYYI ITLVVVNGNVVTKPSRSITVLTKPLPVTSVS IYDYKPSPETGVLFE I HYPEKYNVFTRVNISYWEGKDFRTMLY KDFFKGKTVFNHWLPGMCYSNITFQLVSEATFNKSTLVEYSGVSHEPKQHRTAPYPPQNISVRIVNLNKNNWEEQSGNFPEESE MRSQDTIGKEKLFHFTEETPEIPSGNISSGWPDFNSSDYETTSQPYWWDSASAAPESEDEFVSVLPMEYENNSTLSETEKSTSG SFSFFPVQMILTWLPPKPPTAFDGFHIHIEREENFTEYLMVDEEAHEFVAELKEPGKYKLSVTTFSSSGSCETRKSQSAKSLSF Y ISPSGEWIEELTEKPQHVSVHVLSSTTALMSWTSSQENYNSTIVSVVSLTCQKQKESQRLEKQYCTQVNSSKP I IENLVPGAQ YQVVIYLRKGPLIGPPSDPVTFAIV (4) PTGIKDLMLYPLGPTAVVLSWTRPYLGVERKYVVEMFYFNPATMTSEWTTYYEIAA TVSLTASVRIANLLPAWYYNFRVTMVTWGDPELSCCDSSTISFITA(3) PVAPEITSVEYFNSLLYISWTYGDDTTDLSHSRML HWMVVAEGKKKIKKSVTRNVMTA I LSLPPGDIYNLSVTACTERGSNTSMLRLVKLE (2) PAPPKSLFAVNKTQTSVTLLWVEEG VADFFEVFCQQVGSSQKTKLQEPVAVSSHVVTISSLLPATAYNCSVTSFSHDSPSVPTFIAVSTMV (1) TEMNPN

RKKHLQMARECGAGTFVNFASLERDGKLPYNWRRSIFAFLTLLPSCLWTDYLLAFY INPWSKNGLKKR KLTNPVQLDDFDAYIKDMAKDSDYKFSLQFEELKLIGLDI PHFAADLPLNRCKNRYTNILPYDFSRVRLVSMNEEEGADYINAN YI PGYNSPQEYIATQGPLPETRNDFWKMVLQQKSQI IVMLTQCNEKRRVKCDHYWPFTEEPIAYGDITVEMISEEEQDDWACRH FRINYADEMQDVMHFNYTAWPDHGVPTANAAES ILQFVHMVRQQATKSKGPMI IHCSAGVGRTGTFIALDRLLQHIRDHEFVDI LGLVSEMRSYRMSMVQTEEQYIFIHQCVQLMWMKKKQQFC I S DV I YENVSKS
\end{abstract}

>PTPRO.mouse Ptpro-201 ENSMUST00000077115 [Mus musculus]

MGHLPRGTLGGRRLLPLLGLFVLLKIVTTFHVAVQDDNNIVVSLEASDIVSPASVYVVRVAGESKNYFFEFEEFNSTLPPPVVF KATYHGLYYI ITLVVVNGNVVTKPSRS ITVLTKPLPVTSVS I YDYKPSPETGVLFE I HYPEKYNVFSRVNISYWEGRDFRTMLY KDFFKGKTVFNHWLPGLCYSNITFOLVSEATFNKSTLVEYSGVSHEPKOHRTAPYPPRNISVRFVNLNKNNWEEPSGSFPEDSF IKPPQDSIGRDRRFHFPEETPETPPSNVSSGSPPSNVS SAWPDPNSTDYESTSQPFWWDSASAAPENEEDFVSALPADYDTETT LDRTEKPTADPFSAFPVQMTLSWLPPKPPTAFDGFNI LIEREENFTDYLTVDEEAHEFVAELKEPGKYKLSVTTFSSSGACETR KSQSAKSLSFYISPTGEWIEELTEKPQHVSVHVLSSTTALMSWTS SQENYNST IVSVVS LTCQKQKESQRLEKQYCTQVNSSKP VIENLVPGAQYQVVMYLRKGPLIGPPSDPVTFAIV (4) PTGIKDLMLYPLGPTAVVLSWTRPILGVFRKYVVEMFYFNPTTMTS EWTTYYEIAATVSLTASVRIASLLPAWYYNFRVTMVTWGDPELSCCDSSTISFITA (3) PVAPEITSVEYFNSLLYISWTYGDA TTDLSHSRML HWMVVAEGRKK I KKSVTRNVMTA I LS LP PGD I YNLSVTACTERGSNTSLPRLVKLE (2) PAPPKSLFAVNKTQT SVTLLWVEEGVADFFEVFCQQLGS GHNGKLQEPVAVSS SVVTISS SLPATAYNCSVTSFSHDTPSVPTFIAVSTMV (1) TEVNP NVVVISVLAILSTLLIGLLLVTLVIIRKKHLQMARECGAGTFVNFASLEREGKLPYSWRRSVFALLTLLPSCLWTDYLLAFY IN PWSKNGLKKRKLTNPVQLDDFDSYIKDMAKDSDYKFSLQFEELKLIGLDI PHFAADLPLNRCKNRYTNILPYDFSRVRLVSMNE EEGADY INANYI PGYNS PQEYIATQGPLPETRNDFWKMVLQQKSHI IVMLTQCNEKRRVKCDHYWPFTEEPIAYGDITVEMVSE EEEEDWASRHFRINYADEAQDVMHFNYTAWPDHGVP PANAAES ILQF'VFTVRQQAAKSKGPMI IHCSAGVGRTGTF IALDRLLQ HIRDHEFVDILGLVSEMRSYRMSMVQTEEQYIFIHQCVQLMWLRKKQQFC IS DV IYENVSKS

>PTPRO.chicken PTPRO-201 ENSGALT00000037455 [Gallus gallus]

MLPQFTIAGHGSEPGGGRRGAAARGRGAEEPQPPPPGRSRGPRP PRS PMPDRRRRGARGGP GARSCLLWLFVLLKNAEPFQVIV QEDNC IVVS LEAFDVVS S SSVYVVKIAGESKNYFFOFEEFNSTLPAPVIFNAKYHGLYY IVTLLVVNSNMVSKSARS ITVLTKP LPVSSVSIYDYKP S PETGVLFEIQYPEKYNVFTRVNISYWEGKDYRTMLYKDFFKGKTVFNHWLPGICYSNITFQLVSEATFNK STLVEYSGVRHE PKQHRTVPYPPRNISVQ IVP INRNNWEEHSGNFAEES FMGQQE I I SKEKLSRFPS DP PE I PVVNTTAAWPDY RSNSTEYETTSQPYWWSNEAESSEGEEEFVNAVPRDYGASEANASGKLTAEPAPFLPVQMVLTWLPPKPPTAFDGFH INIEREE NSTELLTVSEDTHKFVAELKEPGKYKLSVTTFSSSGSCEVRGSQSAKTLSFY ISPTGEWIEELTEKPQHVTVTVLSSTTALTSW TASHESGSNGTIVSVVSLTCQKQKESQRLEKHYCTEVNSS S S IENLVPGAQYQVVVYLRKGPLVGPP SDPVTFS IV (4) PTGI KDLTLYPLGPTAVVLSWNRPFLGVFRKYVVEMFY FNPSTMTSEWTTYYEIAAT IS LTASVRIANLLPAWYYNFRVTMVTWGDPE LSCCDSSTISFITA (3) PVAPEITS IEYYNHLLYVTWTYGGDGTDLSHSRMLHWMVIAEGKKKIKKSVTRSVMTAVLSLPPGDI YNLSVTACTERGSNTSLPQLVKLE (2) PAPPKSLFAVNKTQTSVTLLWVEEGVADFFEVYCQQAGSNQEVKVQEPVTVSSHVVT ISSLTPATSYNCSVTTFS HNS P SVPTYIAVSTMV (1) TEMNPNIVVISVLAI LS ILL I GLLLVTLVVLRRKHLQMARECGAGTE VNFAS LERD GKLPYNWRRSVFAFLTLLPSCLWTDYLLAFY INPWSKNGLKKRKLTNPVQLDDFDGYIKDMAKDSDYKFSLQFEE LKLIGLDI PHFAADLPMNRCKNRYTNILPYDFSRVRLVSMNEEEGSDY INANY I PGYNSPQEYIATQGPLPETRNDFWKMVLQQ KSQI IVMLTQCNEKRRVKCDHYWPFTEDPIAYGDITVEMLSEEEHTDWVYRNFRISYADEVQDVMHFNYTAWPDHGVPTANAAE SILQFVQMVRQKSVKSKGPMI IHCSAGVGRTGTF IALDWLLQHIRDHEFVDILGLVSDMRSYRMSMVQTEEQY IFIHQCVQLMW QKKKQQFCISDVIYENVSKS

>PTPRO.frog Xenopus Tropicalis ptpro-202 ENSXETT00000048747 NM_001113813.1 PARTIAL Xenopus tropicalis 
TFQVALTQDNCLVISLES PDVLSTKSVYLVKITGESKNYFFQFEEFNSTLPSPLVFNATYHGLYYI ITLIAVNSNMASRS IKS I TVLTKPLPVSHVVIHDYRPSPQTGVVFEVQYPEKFNVFSRVNISYWEGSHFRTVLYKDFFKGKTVFNHWLPGTCYGNITLQLVS EASFNKSTLVEYSGVGHRPQFHRTVPFPPRNISVQILRLNLSFTDLLNGKNNEDSLKKYQDFTEPESSAEMFEDQNERSTFPSI YNSTDPQYKNNTLPEATSQPFWWANETLPPDTDGFVNGVLPDYEDPPVGSSVPNSSSLSFKLLISWLPPKAPTAYDGFNIYIFR DGNASAVTAVDESAHEFVAEMREPGKYWVEITSFSSTGSCEIRESSAAKAISFYIKLLASAGELTPWGPRSTEPYKLLLYSDVI I I I LGLYELHKSHIQISP SCSLTCPKQKESQRLERHYCKEVNS PKPVDH IETPNRVLQKVGYLRVTPRKVAGQISDPPHFLVN PPGVTDLLLYPLGPSAVVLGWRRPYLGAFRKYMVEMFYFNPAAMS SEWTPYYEVAATVSLTASVVPFAPRVPCAPHVPFAPRVP FAPRVPCAPHVPFAPRVPCAPHVPFAPRVPCAPHVPCALXEVRGDINI SRRILYTALFPSGSHEMLOGVSRSGTATVISVPPGD IYNITVTACTERSSNTCAFTLLQSDPAPIRTLFAVNKTQTSVTLLWVEEGIADYEEVSCWQNREPGAETNQKDPVAVFSHIVTI SSLQPDTSYNCSVTSISHRTP (1) SAPTELTVSTLVAELN

NFASLEKDGKLPYNWRRS I FAFLTVLP SCLWTDYLLAFY INPWNKNGLKKRKLT PVQLDDFDLYIKEMSKDSDYKFSLQFEELK MVGLDI PHFAADLPSNRGKNRYTNILPYDCSRVKLISLEADEGADY INANYI PGYSAAQEY IATQGPLPETRNDFWKMILQQKC QVIVMLTQCNEKRRIKCDHYWPFTPEPVNYGDITVEMASEEEQSDWAQRVFRVSYADETQCVTHFNFTAWPDHGVPTVNAAESV LQFVQVVRQKALKSKGPVTVHCSAGVGRTGTF IALDWLMQH IRDHEFVDILGLVSELRSHRMSMVQTEEQFVF IHQCAQLMWKK KRQQFRISDVIYENVSKS

>PTPRO Xenopus laevis gi|1050385751|gb|OCT87944.1| hypothetical protein XELAEV 18016573mg []

MPPPAAQPVSHPSRQTRDCDISECPPRRSRISECDREPGRRRRRRRPARISVRPGGTERQDPSLHFPFNPRGQRAAADPLQRLR PPRPARVMI LLTCLLLFIFLQDGSTFQVAVTDDNCLVISLEPS DVLNTKSVYLVKITGESKNYFFQFEEFNSTLPSPLVFNASY HGLYY ILTLIAVNSKMTSRSSKS ITVLTKPLPVSHVT I HDYKPS PQTGVVFOVQFPEKFNVFSRVNI SYWEGRHFRTVLYKDFF KDKTVFNHWL PGTCYSNI TLQLVSEASFNRSTLVEYSGVGHQPQS HRTVPFP PRNI SVQI LPLNHS ITDLLNGKNNEVTVTKYQ DFPESSAEMFDDQNERSTFIQLYNLTDPQYKNNTFPEATAQPFWWASETIPPDTEGFVNGMLPDYEDP PVRSSEPPSPSPNFKI LISWLPPKAPTAYDGFNIYIFRDGNASVVSSVDESTHEYVAELVEPGKYFVEITSFSSTGSCEIRESSPAKAISFYISPTGDWF EEMTEKPRDVAVK IVNSTNALVSWASSPDISPIVSVISLTCSKQKESQRLEKHYCKEVNVS SNI I GNLVPGAQYKVVVYFQRPP LIG (4) PPSDAVLFA IDPTGIKDLVLFPLGPSAVI LSWSRPYLGVERKY IVEMFYFNPVAMSSEWTPYYEIAATVSLTASVKVT DLLPAWYYKFRVTMATWGEP (3) QLSCCDTTVSFVTAPVAPQITSVEYSYGLLHVTWAYADGSFDLSHSRIVHWQVIAEGKKRI KKSVSRSGMATVLSVPAGDIYNITVTAFTERSS (2) NTSLPHLVKLDPAP IKTLFAVNKTQTSVTLLWVEEGITDYFQVSCWHN VETGTNKNDKEPVTVFSHVVTISSLEPATSYNCSVTSISHRTSS (1)APTFITVSTLVTELNPN

MLIVIRRKHLQTSRDCGAGTFVNFATLEKDGKL PYNWRRS I FAFLTVLPSCLWTDYLLAFY INPWNKTGLKKRKLTNPVQLDDF DSY IKEMSKD SDYRFSLQFEELKMVGLDI PHFAANLPSNRGKNRYTNILPYDFSRVKLISLDADEGADY INANYIPGYSAVQEY IATQGPLPETRNDFWKMILQQKCQVIVMLTQCNEKRRIKCDHYWPFTSEPVNYGDITVEMVSEEEQSDWAQRVFRVSYGDESQC VTHFNFTAWPDHGVPTVNAAESVLHFVHVVRQKALKTKGPITVHCSAGVGRTGTFIALDCLMQH IRDHEFVDILGLVSELRSYR MSMVQTEEQFVFIHQCVQIMWKKKRQQFRISDVIYENVSKS

\section{>PTPRO.fish zebrafish ptpro-204 ptpro-204 ENSDART00000122021 Danio rerio}

MSSARARFFFIIFIFIOSAAGFRVOLODGVRMLMS LDDGDI GPGVSEYAVRVSGEPLTHTLLFOOAADAHTLPEPLLFNASYHG LLYS IS LMTDGRTHTTRS I ITQPLPLDSVEMWDYAPS PETAVVFQ IRS P DRNIFTRVN ISYTEGHQRRYMLYKDFLHGKTVFKH WLSGVCYSNITFQLISEASVNRSALLSRSDITHNPQQHRTVPNPPLNVSLKI LHLSGRGP PGPVLTGA I LKNSHNASVVRRERD LPEYEEQEPTSDTLNTESNLTHTQLNQSESENESESEPVTAEPTLNSSTQSLWAWQTVSPAPTEEEEEGFVNALVPEYEDSNEP GSALGIPLEPAVMPAVMPTPLAPVLLQLRWSPPAPHTAYDAFNIYIYRNGNSTETATVDENTHEFLAELSESGTYRIHVTTLSS AGDCEARESSANTAFTFYLSP SGEWMEQPQERPQAVSVKMLDS STAAVSWAPSTHTYNGS LISVQSLTCLRPS ISQRMELNYCS EENITSDI ISSLTPGAQYRVVVYHTNGPLVSPASEPVI IDIE (4) PTGVRDLVVYPLSPSAVILSWQRPYNVAFRKYVLQTFFF NSATQTAQWSTYYEIAATASVIASVRVTDLLPAWFYNFRVTMVTWGDPPLSCCDTSTVSFITA (3) PEAPHISSVEFSHGSVFV RWTYGDLFTDLTHSRMLHWQVVAEGKKSARRRFSVDVTRSVMKASLALPAGD I YNLTVTACTERSCNTSAPH IMKLD (2) PAPP RSLYAVNASDTSVTLIWAEEGVVDHYLITCRALGAHAEQKKVREPLVTSAHVLTVSGLQASTTYNCSVRSSSYSSSSDPVHITV STTV (1) REMNPSVAA I SALAVLSVLL I S LLVLFLLVLRKKHMELSRECGAETFVNFASFERDGKLPYNWRRSLFAFLTLLPSC LWTDYLLAFY INPWSKTALKKRKLT SPVOLSDFEAYLKDMGKDSAYKFSLOFEELKSVGLDLSHEAADLPINRPKNRYTNILPY DFSRVKLISLHNDEGSDY INANYI PGYNSPREY IATQGPLPDTRNDFWKMVLQQKVHI IVMLTQCNERRRVKCDHYWPFSDEPV AYGE ISVEMLAETDSPEWTIRSFRLAYADETQDVLHFNYTSWPDHGVPTVNAIESILQFVQIVRQQVNRSKGPIVVHCSAGVGR TGTFISLDRLMQHIQEHEYVDVLGLVSDMRSHRLSMVQTEEQYVFIHQCVLLMWKKKKQQSHTS DV IYENVSKS

\section{PTPRH}

>PTPRH.human ENSP00000365528 [Homo sapiens]

MAGAGGGLGVWGNLVLLGLCSWTGARAPAPNPGRNLTVETOTTSS I SLSWEVPDGLDSONSNYWVQCTGDGGTTETRNTTATNV TVDGLGPGSLYTCSVWVEKDGVNSSVGTVTTATA (8) PNPVRNLRVEAQTNSS IALTWEVPDGPDPQNSTYGVEYTGDGGRAGT RSTAHTNITVDGLEPGCLYAFSMWVGKNGINSSRETRNATTA (7) HNPVRNLRVEAQTTSS ISLSWEVPDGTDPQNSTYCVQCT GDGGRTETRNTTDTRVTVDGLGPGSLYTCSVWVEKDGVNSSVEIVTSATA (6) PNPVRNLTVEAQTNSS IALTWEVPDGPDPQN STYGVEYTGDGGRAGTRSTAHTNITVDRLEPGCLYVFSVWVGKNG INSSRETRNATTA (5) PNPVRNLHMETQTNSS IALCWEV PDGPYPQDYTYWVEYTGDGGGTETRNTTNTSVTAERLEPGTLYTFSVWAEKNGARGSRQNVS ISTV (4) PNAVTSLSKQDWTNS TIALRWTAPQGPGQSSYSYWVSWVREGMTDPRTQSTSGTDITLKELEAGSLYHLTVWAERNEVRGYNSTLTAATA (3) PNEVTD 
LQNETQTKNSVMLWWKAPGDPHSQLYVYWVQWASKGHPRRGQDPQANWVNQTSRTNETWYKVEALEPGTLYNETVWAERNDVAS STQSLCASTY (2) PDTVTITSCVSTSAGYGVNLIWSCPQGGYEAFELEVGGQRGSQDRSSCGEAVSVLGLGPARSYPATITTIW DGMKVVSHSVVCHTESAG (1) VIAGAFVGILLFLILVGLLIFELKRRNKKKQQKPELRDLVFSSPGDI PAEDFADHVRKNERDS NCGFADKYQQLSLVGHSQSQMVASASENNAKNRYRNVLPYDWSRVPLKPIHEEPGSDY INASFMPGLWSPQEFIATQGPLPQTV GDFWRLVWEQQSHTLVMLTNCMEAGRVKCEHYWPLDSQPCTHGHLRVTLVGEEVMENWTVRELLLLQVEEQKTLSVRQFHYQAW PDHGVPSSPDTLLAFWRMLRQWLDQTMEGGPPIVHCSAGVGRTGTLIALDVLLRQLQSEGLIGPFSFVRKMRESRPLMVQTEAQ YVFLHQCILRFLQQSAQAPAEKEVPYEDVENLIYENVAAIQAHKLEV

\section{>PTPRH.mousePtprh-202 ENSMUST00000166650 [Mus musculus]}

MARAGGNCGVWRSLVLLGLYGCSVVRAAGTSVTVDRHAPASSYEFSMWVEKDGVSSSPQI PVTTAAPNPVRNLRVEGQNNIS IS LSWEPPDQSSLQGLTYWTQCSRHGGQTETRNTTDTSVTVDGLDPGSSYECSVWVEKDGLYSKNETLNTSTA (6) PNPVRNLRVE GQNNIS ISLSWEP PDQPSLQGLTYWAQCSRHGGQTETRNTADTSVTVDGLDPGSSYECSVWVEKDGVYSTNETLNTSTA (5) PN PVRNLRVEGQNNIS ISLSWEPPDQP SLQGLTYWAQCSRHGGQTETRNTTDTS ITVDGLDPGSSYECSVWVEKDGVYSTNETLSN TTA (4) PNPVRNLRVKSQNNFSISLSWEP PDQP SLQGLIYWAQCSRHGGQTETRNTTDTSVTVDGLDPGFLYKCSVWVEKDGVY STNETLNTSTV (3) PISASNPVRNLRVEGQNNFSISLSWEPPDQSSLQGLTYWAQCSRHGGQTETRNTADTSVTVDGLDPGSSY ECSVWVEKDGVYSTNETLNTSTV(2) PAAVNITSCISTSGGYGVLLTWSCPSGGYESFEVKVGRKWRSENGSLCGKGVTVSDLE PAQSYTATVTTVFKDLKAQSLSTTCHTESAA (1) I IAGAIVGILLIFILVGLIIVEIKRRRKKRQPKEVPKDLVCSCPGDILAK DFADHVRENEKDSNCGFAEEYQQLALEGQGQSQITASALENRSKNRYRNVLPYDWSRVPLQPLQEEPGSDYINASFMPGLWSPK EFIATQGPLPNTVGDFWRMVWEQQSHTLVMLTNCMESGRVKCEHYWPLDAQPCIHGQLQVMLISEEASENWTVRHLQLFHMKEQ QTLSLRQFHYLAWPDHGVPYSPDPLLAFRKMLRQWMDQTTDGGPPIVHCSAGVGRTGTLIALDVLLRQLECEGLVGPFSFVKKM RESRPLMVQTEAQYVFLHQCILKSLQKPAPALVPEEAMYENVASLVYENASAIMAHESEFSASGC

>PTPRH.frog xenopus XP 004916623.1I [Xenopus (Silurana) tropicalis]

MEYDEALTGICNVQILLKAMRTḠCYVTLGYVPIRSHRFQKRLLSGVPSPVQSVSVSNITTTNVTLSWVPPNDINKDTYTYS ITV YNGSSPWDWPKNISGTSTQVTGLIPGVFYTFSVFTVTSNGTMSSQAGSTSGTTVPSPVQSVS ISNIMTTNVTLSWVPPNDINKD TYTYS ITVYNGSTSWNGPTNISGTSTQVTGLIPGVYYTESVFTVTSNGTKSSQAGSTSGTTV (7) PSPVQSVS ISNIMTTNVTL SWVPPNDINKDTYTYSITVSNGSSPWNGPKNISGTSTLVTGLIPGVFYTFSVFTVTSNGTMSSQNGSTSGTTG (6) PSLVTTVS I IKQTNNSVTISWTPPQDINQATYMYSINVTNSAGSWNVNS STNVSSKEVLDLLPGVTYTFCVYTVTSDGTKSSDCSPDSSTTR (5) PTLVKDVTVKSQTTDSVTLSWTTPNDINKGSYYYLITTACGTTTCNVNSTVNANGATVASLLPGLTYQFSVYTVTSDGTNS SAFASTSGTTA (4) PNAPTSVSGNPVNTTALSVNWTP PNDLNKNNYTYTVFWQRNNESLNQSITSNTTI IGGLTAGNQYNVSVI SVINNVPSVKAVAYLQTN (3) PEVPGGFKIVTITNSSVNVSWTIPSGGSFSGIEINAVSGTSNLTKQFTGPSSEGILDGLIPGT AYNLILRSFSSYPSGSSGRISRRATTNNI ITYSNPI IEKVQLD(2) PNTVTGITCTKVGGGYQLKVAFVCPSGSYSS IKILADG NEKETIPAQNCSQGATVQSLQPATKYIITVETVADSRQVSDFIICYTDNV(1) GVIVGSIFGVLLFLLVVGLIAYFVLRKRRGS KNPFKKERGNMQK INIVRMP SVMKSAFPDYYQROHADSDFGFAEEYQQLSNVGINQSKLAAELSENRSKNRFTNVLPYDHSRVR LNRIDADETSDY INANYMPGYNSSKEFIASQGPLPNTSADFWRMIWENQVST IVMLTNCMENGRVKCEHYWPLDYTPCTYGDIT VTVTSEMILPDWTVRDFTLKHAKQQGNKHARHFHFTVWPDHGVPENTTTIVEFRNLVREYMDLKRSSGPTVVHCSAGVGRTGTL IALDYLIQKMEKEQRIGIYSFVQKMRQNRPIMVQTESQYVFLNKCMLDLIQNPPDEN IYENQIGGDLIYENAS

>PTPRH.fish ptprh-202 ENSDART00000114768 zebrafish Danio rerio

MELVLAFALLCVLSVSGQATLPDVNDVRITHRTETELTFQWDKAGGSNSYSYILRRDGIDSDIITAGTVNQVTYKVSDLSSGTN YNWTLFTVFGDQRSEGKNFSAATVPPNVESVSVIDQSETTITLQWKKDKAEYSYNLTYGDKVDTPPSPQSTVEYTVTSLSPGTN YSFTLYTVFEGLKSSGFSFFAVTV (5) PSKITGFSVIGRSETALTFQWTKEKDEYTYELDKS DANTQISFTDTTVVCLITSLSP GTEYSYNLYAVFEGVKSQAKGISEVTV (4) PSNVENVSVVEANDTDVILQWNAVPNEQNKYNYI LKYNDVEESVDFTTENEIKH HVSGLIPATNYNFTLHTEFYSQNSTGYNFNHTTF (3) LSSVTEVRVNRSLTELTVEWNKLNQNNVYNYTLLRSDGAEIDFTGSA VVGDVIKHKYSSLKPGTVYSFTLFTVSNNVRSSEHSFKSITA (2) LDCASFNWKVTNTS IVADVYGCTYVTAQNSSGSGKNASI EGDKVNLRDLYPGESYTVSLFYNLESKMLTQCSQRLTLVPYVVSNLKCEYHSGGYGLAVMWDYPDGVVDVVEVDVNKKSFNHSH GSDEPTQQLVTGLQAAQWYKVKATSFSGARRSETILINCQTDPAG (1) V

ADLKVSKKNYKTTACDKFPEHFRNMSRDDNRAFSA FYDLSVGVEOSKVAAIIPENKDKNRFSNVIPYDTSRVHITINKAGDS DYINANYMPGYGNASREYIAAQGPLPSTVNDFWRMIWEKKSSTIVMVTNCTEGGRVKCEQYWPLDYTPCLYENLLVTVKSENKS QSWTLREFNVKNKMTSETRTVRHFHFTAWPDHGVPRGTEELIQFRDLIRQHIESHFSTGPTVVHCSAGVGRTGTLIALDVLLQQ LNREKAVGVAAFVQEMRLNRPLMVQTESQYVFLHQC ILDSLQTKFVQQSE P I YONTDT I YVNAMALKDFENS SHT

\section{PTPRG}

$>$ PTPRG.HS ENSG00000144724

MRRLLEPCWWILFLKITSSVLHYVVCFPALTEGYVGALHENRHGSAVQIRRRKASGDPYWAYSGAYGPEHWVTSSVSCGGRHQS PIDI LDQYARVGEEYQELQLDGFDNESSNKTWMKNTGKTVAILLKDDYFVSGAGLPGRFKAEKVEFHWGHSNGSAGSEHS INGR 
RFPVEMQIFFYNPDDFDSFQTAISENRI I GAMA I FFQVSPRDNSALDP I I HGLKGVVHHEKETFLDPFVLRDLLPASLGSYYRY TGSLTTPPCSEIVEWIVFRRPVPISYHQLEAFYS IFTTEQQDHVKSVEYLRNNFRPQQRLHDRVVSKSAVRDSWNHDMTDFLEN PLGTEASKVCSSPPI HMKVQPLNQTALQVSWSQPET I YHPP IMNYMI SYSWTKNEDEKEKTFTKDSDKDLKATISHVSPDSLYI FRVQAVCRNDMRSDFSQTMLFQANTTRIFQGTRIVKTGVPTASPASSADMAPISSGSSTWTSSGI PFSFVSMATGMGPSSSGSQ ATVASVVTSTLLAGLGFGGGGISSFPSTVWPTRLPTAASASKQAARPVLATTEALASPGPDGDSSPTKDGEGTEEGEKDEKSES EDGEREHEEDGEKDSEKKEKSGVTHAAEERNQTEPSPTPS S PNRTAEGGHQT I PGHEQDHTAVPTDQTGGRRDAGPGLDPDMVT STQVPPTATEEQYAGSDPKRPEMPSKKPMSRGDRFSEDSRFITVNPAEKNTSGMISRPAPGRMEW

RGCNKIKSKGFPRRFREVPSS GERGEKGSRKCFQTAHFYVEDS S SPRVVPNES I P I I I PDDMEAI PVKOFVKHIGEI YSNNQHGFSEDFEEVQRCTADMNITAEHSNHPENKHKNRYINILAYDHSRVKLRPLPGKDSKHSDYINANYVDGYNKAKAYIAT QGPLKSTFED FWRMIWEQNTGI IVMI TNLVEKGRRKCDQYWPTENSEEYGNI IVTLKSTKIHACYTVRRFSIRNTKVKKGQKGN PKGRQNERVVIQYHYTQWPDMGVPEYALPVLTFVRRSSAARMPETGPVLVHCSAGVGRTGTYIVIDSMLQQIKDKSTVNVLGFL KHIRTQRNYLVQTEEQY IF IHDALLEAILGKETEVS SNQLHSYVNS I L I PGVGGKTRLEKQFKLVTQCNAKYVECF SAQKECNK EKNRNSSVVPSERARVGLAP L PGMKGTDY INASY IMGYYRSNEF I I TQHP LPHTTKDFWRMIWDHNAQ I IVML PDNQS LAEDE F VYWPSREESMNCEAFTVTLISKDRLCLSNEEQ I I IHDF ILEATQDDYVLEVRHFQCPKWPNPDAP ISSTFEL INVIKEEALTRD GPTIVHDEYGAVSAGMLCALTTLSQQLENENAVDVFQVAKMINLMRPGVFTDIEQYQF IYKAMLSLVSTKENGNGPMTVDKNGA VLIADESDPAESMESLV

\section{PTPRF}

>PTPRF.HS ENSG00000142949

MAPEPAPGRTMVPLVPALVMLGLVAGAHGDSKPVFIKVPEDQTGLSGGVASFVCQATGEPKPRITWMKKGKKVSSQRFEVIEFD DGAGSVLRIQPLRVQRDEAIYECTATNSLGEINTSAKLSVLEEEQLPPGFPS I DMGPQLKVVEKARTATMLCAAGGNPDPEISW FKDFLPVDPATSNGRIKQLRSGALQIESSEESDQGKYECVATNSAGTRYSAPANLYVRVRRVAPRFS I PPSSQEVMPGGSVNLT CVAVGAPMPYVKWMMGAEELTKEDEMPVGRNVLELSNVVRSANYTCVAISSLGMIEATAQVTVKALPKPPIDLVVTETTATSVT LTWDSGNSEPVTYYGIQYRAAGTEGPFQEVDGVATTRYSIGGLSPFSEYAFRVLAVNSI GRGPPSEAVRARTGEQAPSSPPRRV QARMLSASTMLVQWEP PEEPNGLVRGYRVYYTPDSRRP PNAWHKHNTDAGLLTTVGS LLPG ITYSLRVLAFTAVGDGPPS PTIQ VKTQQGVPAQPADFQAEVESDTRIQLSWLLP PQERI IMYELVYWAAEDEDQQHKVTFDPTSSYTLEDLKPDTLYRFQLAARSDM GVGVFTPTIEARTAQSTPSAPPQKVMCVSMGSTTVRVSWVPPPADSRNGVITQYSVAYEAVDGEDRGRHVVDGISREHSSWDLV GLEKWTEYRVWVRAHTDVGPGPESS PVLVRTDEDVPSGPPRKVEVEPLNSTAVHVYWKLPVPSKQHGQ IRGYQVTYVRLENGEP RGLPI IQDVMLAEAQWRPEESEDYETTISGLTPETTYSVTVAAYTTKGDGARSKPKIVTTTGAVPGRPTMMISTTAMNTALLQW HPPKELPGELLGYRLQYCRADEARPNTIDFGKDDQHFTVTGLHKGTTYIFRLAAKNRAGLGEEFEKE IRTPEDLPSGFPQNLHV TGLTTSTTELAWDP PVLAERNGRI ISYTVVFRDINSQQELQNITTDTRFTLTGLKPDTTYDIKVRAWTSKGSGPLSPSIQSRTM PVEQVFAKNFRVAAAMKTSVLLSWEVPDSYKSAVPFK ILYNGQSVEVDGHSMRKLIADLQPNTEYSFVLMNRGSSAGGLQHLVS IRTAPDLLPHKPLPASAYIEDGRF DLSMPHVQDPS LVRWFY IVVVP IDRVGGSMLTPRWS TPEELELDELLEAIEQGGEEQRRR RRQAERLKPYVAAQLDVLPETETLGDKKNYRGFYNRPLSPDLSYQCFVLAS LKEPMDQKRYASS PYSDE IVVQVTPAQQQEEPE MLWVTGPVLAVILIILIVIA I LLFKRKRTHSP S SKDEQS I GLKDS LLAHSS D PVEMRRLNYQTPGMRDHP PI P ITDLADNIERI KANDGLKFSQEYESIDPGQQFTWENSNLEVNKPKNRYANVIAYDHSRVILTS IDGVPGSDYINANYIDGYRKQNAYIATQGPLP ETMGDFWRMVWEQRTATVVMMTRLEEKSRVKCDQYWPARGTETCGLIQVTLLDTVELATYTVRTFALHKSGSSEKRELRQFQFM AWPDHGVPEYPTPILAFLRRVKACNPLDAGPMVVHCSAGVGRTGCFIVIDAMLERMKHEKTVDIYGHVTCMRSQRNYMVQTEDQ YVFIHEALLEAATCGHTEVPARNLYAH I QKLGQVP P GESVTAMELEFKLLASSKAHTSRF I SANL PCNKFKNRLVN IMPYELTR VCLQPIRGVEGS DY INASFLDGYRQQKAYIATQGPLAESTEDFWRMLWEHNST I IVMLTKLREMGREKCHQYWPAERSARYQYF VVDPMAEYNMPQY I LREFKVTDARDGQSRT IRQFQFTDWPEQGVPKTGEGFI DF IGQVHKTKEQFGQDGPITVHCSAGVGRTGV FITLSIVLERMRYEGVVDMFQTVKTLRTQRPAMVQTEDQYQLCYRAALEYLGSFDHYAT

\section{INVERTEBRATES}

\section{DEUTEROSTOMES}

\section{Tunicates}

>Ciona Ciona Savigny ENSCSAVT00000019606 plus GENSACAN and profiling profiling 
PGESYEIAMISLKGIDQSSAVFVSQTTRAISDMAPPVNFRMTSRGETEITLGWDPAPGVQGVYMVRCEGFQEPCPAEVDI IRPD EKRELRVGRLKPGARYNFTIQTQNGQDISKAKRLSLITV (15) PRTPLSLDTVSRSDTTISLRWDQPTGI LDGYKISYTSRGGQ LEILFVQPGI FEKTLNNLRPGTYYEISLVSTSSEIESEP I I LTEITE (14) LRE IENLS IARLGE TWAF I SWVPGEGGYSGFDY IVQVATVLDKDRSVARNITIQTRPLP PNDVRMDNLTKS SVHVWWREPETFDQFLLVYKDDFNTKNLMTQQTSVDINDLTPGSSY VIDICVIFGSVKSYNVTNEITTY(13) I IAEPGTPTALVLKPVSTTELLTEWE I PLNPNG I RRYI IRFKQNYPHPSTNYTLIE TNQTRYLLGNLEPGAEYVALFAAVNSAGVGNFTESYFFKMPESKPG (12) PVSDLHVS SVNAISATVAWQPPEQPNGEIIRYVI NISTDHLPVLSVDLLKRSNSDKNLCLFDHKSKSDKTALVSVTSS LQNNSVTEALNDEIYFDPLCPVFVNVTSLLPFTLYRFRVT AWTSS GEGDSRIREEMTLEDVPDDP PRHVRLTSATHTSLNVTWFP PNQPNGIVTYTIAVLQS DKMFQSNLTYYDINELRPFTKY GVMVRAETSVGVSPWSEILFADTQQGRPGSVQNLTAQVETSRSVTLQWNPPINPNGVITGYWIFAKVKNVTQLTLWLEGVSL (1 1) NSGVNLRTLQDVPSS PPINLSYYNVSSTAVNITWDEPLEANGVI IKYVVYYMTKDTFLDVTVTERFALIKDLKIFSPYEVI ( 10) RVRPYTLLGDGPASGTLLIHTDEDFPAS PVRNVTSKNITSSVVELSWTPPLI INGRARSYTVYTVVLLNSYNAFNSYSNKT SAFLTNLRVFTAYKVQVACQTKKGLGNILSKTIFIQTEEGQPATPP FNVSYQNLTSTKVRLTWRRPLVPNGI IQFYEISLTSKN NKTIRATTENDVTAVTVDHLTAYSEYTATVRANTKFGDGSQQSHALTIHTLEDVPGSPVTNVTYVNLTSSSIGLEWSLPKEPNG KILKYS IRYSMLEGNMNQYKAVNDTFVELFDLNKYTRYNVSVSAHTVAGEGPPAYISLHTDEDE (9)

\section{NEDKVMTKTTTKPRTVLRNLEKFTEYEVYLTASTKLGNGGKISAIF} PRDVVVKALSSTSISVGWSTPANNGQIQFYTVFYTDKISAVHANGISMNRSTSSSK ILLQITNGTNAVIKDLNKYSDYI VWVSGSTALGDGNQLSDAKKVRTMEDV (7) PDNGVSDLSALA INATTIKVTWQPGI PLTGPTFFH IQYVLNNTPVHDLNF I SFQ PHTEYVIKVYPRVVGVYDAGPVSS IVVTTMESAPSS PVQNLHVVYVTETIVKLKWKPP SEANGVITSYTITVTKQNYNKTKTG ( 6) NETETVIEDLRANSHYNVSVTSSTKIGEGPSTILLFRTKEGVPQDAVTDIQL INLTSDSALIKWLAPRQPNGVITHYTVHYG RNSTIQTNVTEATLRDLHPFQLYWIEVYPWTSAGIGVKPSEKMAIKTKESA (5) PTAPTS LNCSVASNFSVKI SWDLPMRTNGI IRGYNLEYRSMDKNKVKFVQPSQTKQ I SNLKPFANYLIKLRAINLAGKGQWTHCNVTTLEGY (4) ADAPPQLQLKNLSDRS IT IEWSRPLQWNGRFHGYLITYKPPDSCPNPANDTQVCSFTSNLTSVTLEALSKYRAYNISVGSITGRGVGTMRDIFVSTLVGV (3 ) PDSPVRDVHVTVVSSTS INVTWNLPSSYAGPTTYKLYSELVPLYDYFDHTAQYLRVHNHENNSPFSV (2) GLDEDTTYGVIVT TMTTVGELSS IPVS IDTKEDAPSDFPESVGVATVLNNSSSVHVLFKEPLDPNGKLINYTIQHRRLEDSKLQS IVVSVDQLTAPT TEHEPYSVSVSGLLGGRYYQFRVRAATAVGNGPWSLWTNKVLLPTTA(1) PPVPDTWPEVMNKIDDTSTMASVTSSTITVKKPC MFSNENGPIASLSVI IAEDGGNIEAEPTYWSQAFPLQPSP PYKVLVSENPTDYCNTRKRRS INEKGFVI GTSDCP SEINTHCNG PLKSSTDYRVKFRAETSNGLKTDTEFSEIIRTSPSELA

METS I HTNMA I I EASMDDKVQEESVHGNE F SR PVFRDEFPNHVSSLSONKNKGFSLEFDDIRGI PYAGTTAIAENSCNKTKNR TTKLVPFDHCRVKIEGI PAIQGSNY INASYI PGLDSPEQYIATQTPLDHTKKDFWRMLWETGSTNIVMLCGSVNAGKKRCDEFW PKKQTEYFGNLAVQMKEE IRHDEWI IRQF IVTMRDKVRHVTQHHF IKWPQLEQAENSLPLVRFIKNYRLLRDRS INPT IVMCSN GSGRCGVFIGLLRILDNNGRDVDVFGTVAALRKYRPYMVQTLSEYIYLHQCVLKFIDD

>Ciona.1 Ciona Intestinalis genscan and curated by profiling gi|459174018 |ref|XP_002122888.2|] ENSCINT00000033578

MPSLYVISSYEISLTLATSGTAVTKTYTVIAPALTYTVTGLTPGESYTATVQAVSSSVSGSVSTPSLPQRTDVFENQVSSSVVS TVSNLVDVPAEELAVSGEADSLLMTLDTVGEKVELDENGVYEDVTSNVGMAALLP SNKKGRSGLGVES L S INSE PGFRSDQVYI IQGE I PNNVQSF ITLPEEAGMLWVS CNMQ I INACLFSVFGDSGNAKR I IFYAFPNDKLFLS PNNTYYDVNSMVVLSATVEQTSV NNLQEPVIIQLVTNTHAVDAPSGITVDNIKSTEFTVSWTTPTDAISKMTVDIAGTASNENDAVDVVSPHKVINTTAGAAIVGNT EYTITVYAVSSTDATDFKFATNQTTTIFSAPVLTSVTGTNSTIAVDLSWTYDNGGGANAVSEYLIKWDGGGSTGSPTAGSGSTT ATISSLSANTEYTFSITAVSATVRGDTSAPSSATTAGATTTS IDLTWTAPAVGGGKNNVLAYTIQWTGGAGGSKETSGTTDTIS SLSANTAYSFTVAAKSKAGTGEASTPLQAITLPS LPEQPTLTRSTTNPTTVI DVSWPAVTSGTETVDYVVEWTPDEGPAANKAV TGTTTTIDSLVPGQQYNVTVRAHNSAGNSEPSPAARLRTN (11) PPTPTGVSLSNPTNGQTSLKVDWQITKNNFVISSYEISLT PATSGAAVTKTYPANANS PFTYIVTGLTPGESYTATVQAFSSGVSGS ISSASNSQTTV(10) PPTPTGVTLSQSAGDQTTSLKV DWVMPS LYVVSSYNISLI PESSGSAIVTQTFTPSPASTTTDTVTGLTPGESYTATVQAFS SGVSGNNSDASASLRTV (9) SAVP GTPNLYQPTDGSDKTTI LYANWTVPTGVVDSYQLLVYLGSVGGTLVANQTVSTNFANITS L IPGKRYQATVRAFSAGVAGGVSG NSNFAKTN (8) PPTPTGVTLSQPNVNQTTSLKVDWVMIPTSFVISSYI I SLISTTSGAVVNKTYTASAGSAPTYTVTGLTPGES YTATVQAVSSAYDPSTQSDVASSTNTQRTD (7) PPTPTGVQLFQPTVNQTTSLKVDWQITETDFVISSYDITLTPSTSGAAVTK NYPANANSS FTY IVTGLTPGESYTATVQAVSSGVSSSVSTPSNPQRTD (96PPTPTNVTLSKPTVNQTTSLKVDWH ITEAYFVV SSYEISLKITGTTGAAVTKTYSANDASPFTYTVTGLTPGESYTATVQAVSSGVSSSVSTPSNPQRTD (5) PGVPGIPTLSQPTD GSDKTTILYANWTAPTGVVDSYKLLVYRGDVGGTVDANLIVTTNFANITSLI PGKRYQATVTAYSVGVASNVS GNSNYGKTN (4 ) PPTPTNVQLSQPTVDKTTSLVVNWQITPDSSVINYYNIATTAMSTGTITNFYYNLTEKSYTMPGLIPGESYAATVQAFSAKYP PATQSFVGSS LINQRTAKQNPLQLIQYLQQHNLTYVQDGDASTKQTVTLENATTSYNITGLTSGASYTISVVTISNGVRS DEAK QQFSTI (3) TKGVSNLMAQSQTTNS IYVTWNP P PMDVLFTNYFVEYSKMNI F LMPEDHKNENIYLRICITLLLISGYLSYGFYS LWVNVTFTQPKNGANIYIATLTGVTINEQRNGSNSSSPVQVGGLSPGHTYSAVVSASASATILVEGVASSAI I HTIKPEPVTNF RSTEQTSYS I YMSWDLPTLGIFQS QNVTYVPSDS SENAIVVS LGNETTSYNITGLTSGVKYTISVITISNNIESDPATDESMTG PPVATNISITASNTTCVHGTI IYVGRGI I HFYS LTWGFHGSKLVPYHPHITHFNLCGLQAGVEYDINVVSLAGNVSSTYNISTS AYTE (2) PNSVLSVMLSSVS ITE ISVQWTRNSGGVSGYEVIATNSNCVVEANITIAGNTTLSTTLSGLTPGTVFNVQVKALFSA LKSFPVYQSAPTCELVNYTDPEGGDKS IOTNFYETSATLTVLPGYNYTVMVSVTAYGLNSVIOTMTKNTKFNVMIKSRINNVNG VIENSDTFTELYKTNAGPPEEPNITQPVNTGSTSNMNTITIILPANTFNDKQGPIEAFGVYITKETQKKPGPQPDLSRNDSCTN LMTECVAVWYTPAGVANTPSTSSRKKRSYDNP I GNNL I TFVI GS GESTIS PWKTLFVNLPLAVDTPYVVAVAAKTSNNVLVSTN WSQP IRTE (1) TPVPPAPNVGLIVGLTVACVV IVVLI IVVAWFYRKRRKESKKDVSSENQ I P LQQRKTKVIQLAEFLDLLKVMK ADSDFKFSEEYEEFKTVGRDQATVAALLPENRGKNRYTNILPYDATRVKLSAIDDEQGTDYINANFI PGNNNRQREYIATQGPI PGTKEDFWRMVWEQNSRNIVMVTQTVERGKIKCDHYWPFDNEPITVADYTLQMTSESILPEWTIREFKITHGSDTRRIRQFHYT VWPDHGVPDTAETLVKFIRYVRRTIDREAKHSGPTVVHCSAGVGRTGTF IAMDRLLQHLPDNNYVDIFGIVHQMRIHRVFMVQT 
ESQYILIHQMVQDILNRVYDEDDDDQEPVYENTTTISDPIYENAEFNGKQKNGGVVNPALISPSEDDQNKEDSEEEGEEESEEE GEEESEEEGNEENDQNESKLISGSGGPNHDPSVI IKQDAVEETSKERGSKATVV

>Ciona2 Ciona intestinalis XP 002123247.2 partial

LFPHPVADYTNVKTNKTFLVLNQLEPGAGȲYIRIAAINSAGLGTFIKDELIKVMEETPGPVNNLQVSSYNATTVTISWQPPLEP NGEI I YYKLTVYKQNVLATI INFEKKQNENQFNCLLDNAPRDVLSSFMQLLLPNSLLQHNPSTTLTKESGDVTIQALSEI ITSQ TDNNSTKKGKQRTLRS IVTETNEYFEPLCPVAT IVTSLKPFTNYRFNVVGWTAVGEGIGVDVSHTMPQE I PEGP PLNVRVDLAT DSTIRVMWSSPAKPNG IVTYTINIELGNWVYQSNTTYYDVTNLKPFSTYAIKVKAITKVGEGPWSNWI IANTLEGSPESVNSFN VTSKTSRNITLVWEPPLTPNGVLLGYYLIVEENNHIVRSYWLAENNGATREIPSQSDDVIQFDDDILGGKLVDYSMKIKTKTIS DLTPHTAYAFNIFAATVGGNGSQLTLYDVTLEDKPDSPPYNLSYSNITSTSVNISWMEPLKANGVITKYTVYITKDKLQETSVT ERYAVISGLN IYTTYEVYVRATTKVGDGP S SSKMQI HTDEDVPGS PVLDVNFQNISSSVVKVTWS P PLVTNGVILGYTIFYSNF TTYNTTSKTTSVYLEKLKMFANYTVRIASQTSKGLSPMLSGA ITIQTMEGQPSTPPFNLSYTNTTSSVIQLTWLKPLVPNGI IQ FYEITLQTQNNNTIRKTTQSSETSKTIKGLKPFSLYLANVRANTKYGDGGQASATYLIYTMEDAPGSQVMNVSYVNLTSTSVNV SWLQPAEPNGRITKYKIESKLKYTEYIISISAYTMAGEGPREVVKICTDEDE (7)

GI ITLYSVHYRHGNKS LIRTTATPGITLNNLKKFTSY DVYIRASTKFGDGNQTSKIKS FKTLHDA (6) PADPPHDVRVAAISST SINVTWSPPTTPNGLIQFYTVYYQHNPSTVQTKNVTKGMQVV IGNLRKFTNYLVWVTS STALGDGNQMS DAIKVQTLEDV (5) EQKVQELSPRVVNSTAIRVTWLPGQPLTGVTYFMVNVSTKTS PYTEYI ISVTPHVYGVDTPGPTSLVTVTTDEAKPSS PVRNIS IITITQTTVTITFLPPTKSNGVITSYSIMTSLDHKQLVNGSHTKIKLENLKPYKVYNVTVMPYTKMGKGPKAILTFQTLEGVPE DS PQKVQITNITDQSAS LHWE PPTL PNGI IRTYTISYQSNESQRNFNSSKTQVQLTNLAPFTNYSLQLYAWTTRGMGKLPSRVI YFTTHESEPSAPTNLECKI YDNYK I LVTWDLPLLSNGRVIAYKLSHSVLNDTQTYFVKPPQ INQTLQHLQPFTEYFISVNAKTS AGWGP SATCNITTGEGY (4) ATAPQDLNINKTSDVT ITIQWNKPNNVNGRLNGYVIMYI PFDACQPNSG ILNKTRC ILSTNKTW TTLIGMKQHTEYKISVFATTGRGLGAPSNISVRTLVGV(3) PEHSVENLQAIVTSSTTVNVTWESPESFAGPTTYKVEAFHSTT MQRVTSPLLTSSTHSYVMSGLDEDS IYGVTVSTMTSVGQLSSKPVVIMTYEDV (2) PSDFPRDVNVAIVMGNSSSVRVLFKP PR DPNGVLTNYT IQFKRLENSESRNA I IPVSDLVEPT SNNVPYYVT INGLLGGRLYKFRVRAATAVGAGPWSLWTTDVQLPITA (1 PPVPALLPEVI IQQDKAIVTSSSVIVKKPCVFSDDNGPLKSLSVIVAQEGATLDAEPTYWAKAYSEEPSPPYKVIVTEEPQSY CNSRNKRSTQTNNGFVIGTSDCSRLSTSQCNGPLKSNTEYRFKYRAEANNGLMTDTEYSEVFRTNPSFIEA

IF I I LLTI SVALLRRRRRNQ I PNGNES LKTMG P IETMGP I IEEKS KVPVME S SVHSNVAFVMDDSRDMKDTARSP S INEF SRPV AVTDFSLLVEKLSQNKNKGFSSEFDDIRGIPYAGTTSVAEKACNKTKNRTAKLVPFDHCRVKIEGIPAVEGSNYINASYVPGPH SPEQYVATQTPLDHTKKDFWRLIWETGSNT IVMLCNVMEGGKKRCSEYWPKKQTEYFGNLAIQMTKQDVCKDWIVRHFSVTMRD KVRHVTQFQFMNWSVVEQGNPLPLVRFIKSYRTTRHNEYKPTIVMCSNGSGRSGVFIGLDKIVDTMTSYIDVFGTVASLRKYRP FMVQTLTEYIVMHQCIVNFIDDVTIEQPTGDGLEETIF

>ciona.3 Ciona Savigny genscan and curated by profiling ENSCSAVG00000003069

MIGSIWTLVFILGLTCAPC ( ) NAQPAAPTGLSVTSSKSNQLTISWTDTSGTTYKVYVGTGPANVADAVDKTVNSFIATSTTGGS PMSLVDNTE YNVAVYAVDATNTTLVSAASTQPGTTVFVAPTLNPPTNPTSSS IDLSWTAPGNGGGAVTVTGYTITYTGGGGGTQDVTGSSVTSATINMLGSNT AYSFTIAAKSSAGTGDSSTAQPGNTC (13) HTADOCNKHCDEORLAKROVSVSNAKRYVPDKVVLAANTTTATIDT IGLNWNPVSGGVPVTYTV TWSLPTSGSPMTGISANSASVPGLTASTQYTFNVVAVNSAGSSADSGPDNSQYSTL (12) PNAPAAPPSLIPGSNATNMIDVSWTAPTGGNVIV DYVVEWMAHSTTATENTTVTTGTSTTLTLLTAGELYSVNVKARNSAGAGAKS PSASDRTS (11) LKVKHNGTTPQSSAVRQFQKITVQNRI IVV FFDNNLLKS IMLMRYNI LCLGGVRSNLI PGKLYTATVVAYSEGLAGLMS DAS DKIRTSEFLGKFDKLRKPFTTYFTDPPKPTAVTLSOPTSNKT TSLEVTWSMIGTQE IVSSYI INLTPANSGSNAPSVTYIPTETDPTNTIVGGLTPGESYTATVQAVSNNPTPESKSEISEPSAAQRTDPPKPTAV TLSQPTSNKTTSLEVTWSMIGTEYIVSSYI INLTPANSASNAPTVAYRATSTSPTNKIVNGLTPGERYTATVQAVSNGVSGS ISDASPSQRTD ( 10) PTTSLVVTWVKPAGLVDTYGVTLYNEGSLNNPVTGFPKNETGLTATYGNLIPGKLYTATVVAYSGGLAGLMSDASDKIRTN (9) PPKPTAV TLSQPTSDKTTSLEVTWSMIGTEYIVSSYI INLTPANSGSNAPTVTYTATSTSPTNTVVGGLTPGESYTATVQAVSNDPTPEIKSEISVSSAAQ RTD (8) PPKPTAVTLSOPTSVKTTSLKVTWSMITTEYIVSSYI INLTPANSGSNAQIVTYTATSTDPTNTLVGGLTPGESYTATVQAVSNNPTP ESRSEISASSAAQRTD ( 7) PNPPASFTVNPVAANNEITFSFTAPVTPNVFSHFKLTTNATGTTNTTI ISASETTYTFTDAQPNLEHTFYLSAVS GNSPSQTESTSLSATARTS (6) Y

HTMK (5) PEAVTRLLKDSSTTESISITWTLPTAGLFDKQ FMTV (4) TLGVSNLRVATQSTVNIYVLWDAPPASVEFDSYLVEYSVVGMFGMENT INLTAEKKSEFTNITGLNPWEDVSGGSLHHQRGCKGTIC
IRVFCNS ICDDCHFVLPPSPCIGGNCHPTTATHSAKRADLHHNPPPRIHRRLHSHPRPPPAHPHRGEVHQPHPHHHPSHPRPPIHYRNEEILPQ LDLSTNHKESYNNTQHPLTVPYPPTKLQAAANSTSS I IVSWSPP ISGAWWYYVLTYYNSTHPYTIQAPNTTTOLRLTHLLPGTIHFFNISSVAY NTSSEILQISARTK (3) PNPVLSMMATPSNMNDI FVEWLNNTGGVEGYEVQASLSGIVRSNKTI LGNSTLSTTLNGLVAGTFYNISVRVLFASL KSSPLQKQSVPTY (2) PDSVKNLKVLKSSTKPTTELDLTWDQPAGDGENIT (0) IMYTQNPGGLVTNKIASFTDTTKQLTVEPGYNYTVALTVN QFHLSSVTETQVMNTN (1) PAAPVISQALISTTKLVLTWTINGNSENI I IEWGQQGAAVK (0) TRPLPGTTTTQTFDLVKPSTIFNFKISSQIT NVMGATESAE ( 0 ) PHLHTAPTEPNITQS ISVGSATTMTGITI I LPANTFNDKNGP I FYAVYVVKVA QKMGDGTITRS PWNQNF PNQPLAADTE YVVAIAAKTGNDQLVSTNWSTPIRTAPNVGL IAGIVACTVTVT KRRDRKKDEFLDVLKVMKADSDFKFSEEYEEFKTVGRDQ PTTVALLPENRGKNRYTNILPYDASRVKLSAIDDEPGTDY INANFI PVGNNNRQREY IATQGP LPGTKED EWRMVWEQD SRNIIMVTQTVERGK IKCDHYWPFDNEPITVADYTLQMTSESILPEWT IREFKITHGADTRRIRQFHYTVWPDHGVPDTAETLVKFIRYVRRTIDREAKHTGPTVVHCS AGVGRT GTFIAMDRLLQHLVDNNYVDIFGIVHQMRMHRIFMVQTES SY I LVHQMVQD F INRKYDEDD TTEEPVYENATQDD P IYENTSVKHTNG GVVNPALIS PSEDDKNNTEQHDYDCDDEEEEEEVEEEEECSDENSQTNSDGVQVKIVSGNTDDKAPPHVPCMIHSADSTPNMMRSHTTRTRRVD PVITPSRCNSAINPLYSLETTPOEDSSDSEFDDDDDDDIPKA

\section{Equinoderms}


>SeaUrchin1 gi|390362950|ref|XP 790935.3| XP 011667085.1 [Strongylocentrotus

purpuratus ]

MEGNRPCRHLGLS ILGLILICTLQDSVAIDAPDIVTVENEGNTDSLKVTWTPPTGATDQVVTWSPADGSGSDTSFGLSGTATEH TIPGLTPGTLYNVTVTAQNGTDETSDDSEQGRTK (18) PSPVNLTESGATNDS I SAEWTKPSGAI DQYTFSCSEGTEDPSSPIT DVSSNMYEVSCVGLSAAGADYT ITVTSLSGNE ISNESTTTITAL (17) PNMVH IEAGDATNSSFVASWNHPEGEMDSFQVFCYE NDTAPEEISDVGSNSTYMYEVTCESLPTAGTGYQFSVISISGSKNVTSETVLYTSKLGASFGIRLYYFHTSSCNIWLILGPFCN I SLVPMQHNPVTHLQP LHVHLRQHFITL (16) PNPVELTASDSAVDSVRATWLKPAGEVT SYEVQCSNGTVVPP PVQTGDGPFT ASCEDLPTPGDEYTMNVTSVSNGKS SDVKTIALRAL (15) PEAVTLNEERGMVTTTTIAASWTMPS GIVEYYDVFCLVGDPSPV MGNPSGNLTASCVNLTMPGDDYNISVTAVSNGQRSETDTITITAL (14) PLSANLTAGNST INSVSAMWPYPGGLVDVFQVNCS NGTESNVTVLVNQTNPDP SYLVS (13) CDGVWNPGDDYTMVVTSVSNEQENSAS IMLTALPESVMLNEERDRVNTSTITASWTM ANGIVDYYQVSCSNGTASPAQVNPSEDLEASCTRLSTPGDNYTISVTTVSNGQNSAVSTITITAL (12) PSQVSDLAESGATTS SVSATWTKPDGVVSSYTVTCPDGS IQTVPEDNDGSTHNVTCS I LPDPGPGGDH I IMVVTLSGTKASDPATVTITAL (11) PSQV TDLAESGATT SSVSATWTKPDGVVS SYTVTCPDGS IQTVPEDNDGSTHNVTCS I LQDLGPGGDH I IMVVTLSGTKASDPATATI TAL (10) PSQVTDLAESGATTSSVSATWTKPDGVVS SYTVTCPDGS I QTVPEDNDGS THNVTCS I LPDLGPGGDH I IMVVTLSG TKASDPATVTITAF (9) PNSVVLREDGVTTRQ I TVQWVDPVGEEDSF IVDCGDDGSNDITLFPGDATDNTFEATCNTSAAGAPY DITVTSVSGDKESPVTGTFTAAT (8) PDGVTFEEGSASTTAVTVTWIALARADGFRVNCSEGTPSHEGTPSPDSPLPGSATEVT CNGVTPGGNHSVSVITLENNGESPAAVMYVVAV (7) PLSADLAADTSTINSVSAMWPYPGGLVDIFEVNCSNGTERNATLAANE DLNSSYVVSCDGVWNPGDNYSMVVTSLSNGEENSAS I ILTALPVSVNLTAGGSTTSSVTATWDIPGGIVDEFEVECSNGTASTP GNGSDQDYLASCDDVDSPGDNYTMTVKSLSNTQRNSATIVLTAL (6) PESVS LNEA I DMVTTTT ITASWTKPNGIVDYYEVSCS NGDPSPVMGNPSGNLTASCTGLSTPGDDYTISVTSVSNGQRSETDTITITAL (5) PEAVELSEGESNTTVISATWTVRNSVVDT FNITCSDGTASPPS ISVDSSQAGQQLTAYCVGLPTSGEQYDLSVVAISKEKLSAVSTVGVFAL (4) PASVES IAVTEPTTTTVD VQWNLTQCSECVYNYFLLTFQPDSQEAIRVDYMAGVNEYSSQVSGLIQGRSYNFTVVSVSGVGVEDATLKTSEEKSVDQRTG (3 ) DADKRLHGS I I DIVXXXXXXXXXXXXXXXXXGTRASS LINLVAVPEAVGNI SVTQYATTTADVQWNLMQCSDC IYNYFLLTFE PDSQEPIRVDYTTGVNEYSYQVSGLTEGRNYNFTVVSVSGVGVQDATLKTSEEESVDQRTV (2) PATPSDLS IVPGQRELNLVW AYQGDADNFTITVTPNQGIAMFNGDFNDPAAEVTGLTPGTMYDIAIVTASGDERSEP ITQTNRTLPDK (1) PS PVGNPQAEAVD KNTITLTYES PLEPNGDITGYRISYIGTRDGNTDHEFS DFYS PPVLTVTYNDLYPGFSYTFTIIAVNDGGFESDPALTEPVETP QEEPSPVPEDYPYEANTVFSETSTTSFAVVLPDDLFSHENGELLTFAVI ITIDDNDPTVSSTELTYAARAENAY ITAIEIPYPY SPSFGSNRRRRATDPPGTVVI GDGTCAGSQNEYCNGDLDDNTEYYYAFRAYNGMGNVTSSTFGPVKTVKDNTAGIAAGVSVSLI I I IVAVVVVVVFLKRRQPKE P S PRPS LEGRENQGYDGP DHEMRPTRRKTGGSMHRKS HSKP IVLNKFEGHYGRMKADSDFRFTE EYDEIRLVGKDQAIVSALEMVNRAKNRFTNILPYEHSRVKLAALADDSDTDY INANY I PGYNSPREFMACQGPLPGTVDDMWRM IWEKKTSI IVMLTQLVEKGKIKCHEYWPADYNPVTYGSIQVSVQALQQYDHWVIREFS ISQGDE IRKLTQYHFMSWPDHGVPDK TWTMLDFVRTVREAIQKTASDRPIVAHCSAGVGRTGTYIALDRLMQAMQENDYIDIFGIICEM RMQRNHMVQTEKQYIFIHECVMDLLRRGEEEDTGES IYVNLP SNGFKDPDDVNKNLLDQAV

>SeaUrchin3 XP_792888.3 SPU_001461 LOC588094 [Strongylocentrotus purpuratus] MEGGPNAITQLTSSLT̄TSTNITLNWNSA L̄GVVSYYEVAYDP PNGNS PRRTDVRADEPRTLTLSGLFPDSQYTVTVVTWSGTGED ATSSTPFSVMFDTAPILPLQVIVRFVNETEAQLTWGLVLGEDTYI ITVDDSGANP IPSSETVPEGETPLSVRKGLTPGSLYTAN VQTTTATGPDQQFRTS (49) PYPPLDLDVADSTPTS I DVAWAP PRAGGVFDEYILSYSIGDRIRTEVGRFAPDVTTATINNLNT ATTYNIYLVTEANDGGFPQSS DVITREGRTE (48) I PAPGE IVI INRTPTSFRFQWGPS IVPDVTYITTYSPSDGSKLDTLS IR QSDVTGAI PGRLYELTVFVLPGDQQETGLVRTL (47) P SS PGLLAVTAVDYVSVTLTWVPGTGEYTGE I ISYKPTEGITYMDVE EVDQDVRTWKVEGLSPDVSYDFQVVAVSGE HNTTYSEPTRR INTTTLS IAADAFVVLDYNSTS ITVAWRDTNTNI IVNIEPQDD EARNVNVGDRPVHTFTGLNPSTTYTITSLSQSQNLVTQVRTNPAS PSNLEVSKTSTSFSIEWEAAESEVSQYKVDVKCADMSCS FNDVIYPNERLVYELEGLVYLKEYLVTVSSCLDMDGQFAEQMGDEPLESSI I TDDVPDDE IVVQETTTDNITIFYATGNRRGFV EAIYLAGTPAQVGSNSFVTGAVVKKTFDGLVPGTLYTIRITELTNTI IPRTVNVRTR (46) PIAPSAIFGSPTSTVIPIFWFAS PGSISFYEVTYSP PDGTTPAVSRIDASGPLSLDIVTLLPQKVYTVFVRAVTGVDNMPS ISKAVSGMFETL (45) PAQPGEI I LR RITTDTITYTLSEVPSVLQY IVQLNI GTDLQSANPHVLPNVEGEFDELTPGQLYTLVLTVPGTQGDLATIQVRTD (44) PNPPG SVSVELTSVPYSSLLAEWEAPPAGSLSGYEVSVRPADQVLTTMSEVVDSSTTELLLDNLLPEVNYTVEVRT ISAGEGFSSTSDP TEALGRTA (43) PFPEA ILLVTGYDSTT ISVTWRKKEGVDYLAQF IEVGQE I GEDFQGLVQSSSSTTT I LSFRYTDLVPGRNYE LIVRSNDQSEDTIRVSQRTL (42) PSPPMNAAAAPLSAES IRVTWTAATNDFEFYEVTFYPSADSTDSTAMT I PRDQFSVDIDS LLADTEYVFDIRSVSGSGDSLQKSAAITASATTE (41) PI IMRLFSAVENVLLIDFTP IVGIFDDYRYGYRALPSGTITYEPLD SGTSQFGIQGLRSGALYEITLDTRIGSIYTEVDRIQARTRPSAPRLISPVTDFDENSFTLEWLAPSTAEVDFDGFRVIYTPADG ALES PLLLDKTRTSLFLTRLEPGTEYMVTLVSVSGQGSTQQVSFEEDVSVTTA (40) DGVPGGVNIKELTETS IRITWNSALGA TYIPTITPDEGTTRILDTEAAIFEELTPGQLYTLIMTQIGGSSGPPTQQYTL (39) PNAPMDVAISRTTAFELDVSWSPPAEGF YNSFSLTYSRVGTSEVTTIPVPASDAPSVTLPDLLPETSYAVSVVSVVGTINSQPAETSGTTG (38)RAPEGVPVLVERTTTTI S ISWGASTDALATGYQLRIRPEGGTS ILFNLGQA ITTYNFMNLESGTNY I ISLQIFGLSEAPQELPVTTY (37) PQTPGAIQIL RTTQTS ITFAWGSAGGNFDRYS INLYPDGSQTAFLQDVPRGSDLTVSKNYLAPGTLYRIEIYAVTGTTRSALRETTASTA (36) MELLVFITIDAFGVAVSWPNAPDFSNYYLEYDPSNGVP PSPVPTTQDSVFLNS LTPGQIYNFALSSGTPDTGTQSLVRATYVLA (35) PLAPTIE IMILSPTT I LIKI LPASS GVLDYYRVGVTARQTGLGVGQNGVASFN I PREACPEVF ITDLVPGGTYDVEVVAV SGETDSAVSSMDVSLE (34) TMVAGQLYVNDRTTTSLEVVWGAVTDRTFTNYVLNLFLGNTRTDFQSQG INEERKVLFEGLLPG TEYSLNLLVEGDAITQSTILTATA (33) PLTPGS LSFLTVEGQEVTLAWPARNSQVDSYELCWTPSSQTRSPTADATQELLTLE QSDVEYTISLYALVSVGVGDDVKTTRSDAITRFITLS (32) EALEGELNVTDFDSNS I SVEWSEVVSIEFENYRLQATPEGDGS PIFAVI PPEDPRMHTFENLI PGVRYTVNN I LQGSDPVVTRTVSQRTR (31) PNPVSGLTVVDTTSQSVTVNWQQATGS FNDYVV TYTLADEPTTVIYADRI PSDGTRVSVIDGLLPDTSYVVTVYTSSGTDADETTSTEESVPATTT (30)ALPSCSIQVTETTYTEI TVQWGSCAVVVNSYELTIEPNSANAPPGNVPIGGNFEYTFNSLVPGQRYIVGIRGTGSTEPYATDSVYTD (29) PNTPDNLVEV EVSTSTLKVQWDP PVGEYERFEVNLNEQLQGTLRGDQIEAGELPSFTFDALRPGESYSVS I FAVVGSGNLEKRSP PRQNQFTTD (28) S LDAGQLSVIDFDSF S TVVWGAVEGITF DVY I IQFQPPT GNP I YNRVERTQPRESTVTS L IPGQLQT I SL I L IDQVDP I SVIQQRTA (27) PLPPRDLQTQPTVEGSTYS I LLLWSMPVPSA IDQYELTYSPADGLSESF IVLEANDEFYTFRELTPSTTYEF 
RMKTVAGEGDTRTESSEVEMTQRTLSAPLGQLLLRSKTTVTISVTWGVGNTANTI PEGNYVVRITPGDIADVLVNNEERNVDFT LLNPATLYS IRL IEQGNDVS S I DVLTDPVAPNS I SFES IESTSATVTWNRFVGHDVDNFEVWYRQTSS INDYTLAGEVDGDTES IALSGLDPSALYDVQIFSKVTSGELTSKSEPAMDSFTTGALQEGVIEFRDKTTTLIEVLFGTAPLSSEVVQYRVTLTSEEGAST SFNVAPTSEPFSVVTFDGLTPGRLYTVSLQAFGITSTYPTKDVRTF (26) PAPVRGLFPIEIGTTYVLFTWGLPIGDYDSYELS YLKGTAREEEI ISASETNVRIEFLDPNTMYTFNMVTLSAALRSEASDQLFTTTSLAVNEIAVRDVSTTEIEIVWGPEEGVDFWT LTTVPAVSSNILYAQS PTYRFSGLTPGQEYVITVNVNSTSQRTLPEMPQNLESVGMQLFLELOWSPPIGVYDGYRITYTLDGTR TVIPDLPSQPPRYTLRDLDPGTDY IVSVQALSGGLESEPVTRTLTTG (25) VFDQLELSVTTVSNTSIALVWGIYEDRDNLPEI TNYELSITPPDA IS LIRGLDVFRATFERL IPGQEYS IQLKLTTLDEPVSTVVQRTN (24) PDPPAFLRSTRSNFTEIDLAFEAP LSREDFFTLTYEKSDGSDLSPNMRTIAGNEDSVTVEELQPGTSYDFS LTAKSGTPTEFTASQAVEDTFTTRAVPALTVVAAAIT CDTIQIFWAPDPDDMLYRIGIQPGPANDDFRRTTEYIFSGLNPATAYS ISVTVVAFESNSQSEYLPLIQQTRPSGAGA ITVIDK TTTT I DVNWLPAQGNFDSYRVYVTSENGLPKAYPPISATEERRLS ITELDANVLYTVS IETISFFGTQELTSCETSTLDVRTD( 23) ALDANE IAVTAFTSTS ITVIFGKREDATGYSITLRNLDGNAPRDITVNLLPSESSRVTFDTLNPGVNYEITLDSQEDTTIE QRTK (22) PVAPATVLTQNPTVTSFDVIWTQPQGVYHNF LLVITNPDGTKRPE IALGPLDAVREVTELLPGNQYDIQVYATVGT GEDLIRSEPASAQGITN (21) SLGALEVIYIDRTTDSLEVAWGAS PRPNPGVYRVS FNTEGLTDTTPLITQDRNIRGT SLVAGQ LYNVFVVAENYQQDSGI LNVRT I (20) PFVVRNLALTGEPTAISFS ISWNQPAS GMYDGYAVYIAGQDNVERLTSRLDDPDTTE LLIEDLDPNQEYTVSVYAVAGFGQDSEVRSEVTEATVSTV (34) TIGLI I IPVAGSTKFVFTGLTSGTLYRAQVQVTGSSVTSDPLDIRTI (18) PRQPSSFMDTLVTADEISLSWLRPPSGNFDSYE LSYTPENGAKVTLPDVATDETSLVLENLATATEYVILLRTQAGEDTFSEPLELTVTTR (17) SALLALQALPDPTSDVSLDWDR EAGS IQRYMLIYDPNNGDPISP IETG IPGPVAISGLMPGSQYTFRLFSVLTNNVQFLSSSVSHVTT (16) PQPPGNVAVTEEMT STITLSWSAPSQEQFFGYILEYEGTGFSTELGRTPATSVLPRHQESVTLKGLTPGTSYS IKISAFVEYDDVTTRSNIVTKIGTT VS (15) TS S LNIVAKFVNQTS FT IEWQPAVGASSYVTRVSEVTSGVEI S SGT I LNGDYERTEHRLVPGRNYQIEVSATGTGQSD TVEQRTLPSAVSDVDVVPEAAALTLSWDPVFGDVDGYHASYQLKENPYQISDLFYVSDPSNPTIRLTGLSPLSEYI ITLTSFSG SGSDQE IS S STVEASTV (14) NYSPAE IVIETVTS TS IALSWGASTS PTAS SYVIEASDAGNVVHSTSRALSQSRF I I I SNLTP GTLYTIRITVTGSSERDTEIALTV (13) PTAPRS LSTDSISQSIVTVSWSQGLGNVDEYDIYLRQAGESETPAQIVPPSQLTET FENLEPDTYYSMRIVSVVRAQNLEQRS I PAVTNFTTQ (12) PRI INVVETGRNLRLSWANDFGNDILKYQLIYIEDKEGATSNS EFVDPS DAPLVTLMNLMPGQTYTLILTAVDTLGNRAQIASTTYTLK (11) PLRPTFTVNRRS PSAVSLLGNLLGGI LKFFRIRV TPQVTGAGAGTP INIDVLIPVEACPE IELTGL I PNRDYQVTLQAESGVASSDPQIITFTTP (10) PLTQQGELSFGEITPSGFT VYWTPFLGRT SYELSINNQAGLVVPASGDAVQSYVVDGLSSGTEYTVSLVDGGTGAIRTR (9) PLAPGTPLATQVLDT S IQLQW AQSSDGNVAGYEICYSPDDGSSPVYIAGRTTTQYTLEGLDSDTTYIIS ISALTGVPQVKSQKQTIFVTTI (8) ADTTI PVASNF AVTNTQAADHVI I LEWDVPERASYDYFEVSYT P DVGY P SS PVRL PKAAT S LS IMNALADKVYQF S LVS I RGDERTDP PLTTTGQ IA (7) VNNLIVVIGERTTT S LEI LWGASASGSVDEYQVSYVGSTP IVVDGSS DRRVVI PGLSPGTEYTVTVQA INAGAAVGDSM ETASTL (6) HE INGLSAMVTDQAS SMI HLTITP PFED PMRY DGYNVRVRLGGVSS PVVQPVIPVAKSACPE IWIKKLEAATMYE IDIEWYSGLDISEAFRVQTSTVAAAPLDITLDELETDSMKVSWQEGTGSFSNYLVTYS PVGSTQTPMLVAKGEPRVLDLSGLVP GTAYT IMVQQQGQTPAQETHVQLTLPEAINIDSLSVTRSKNALDATWSAPSTGNYDGYRVCHYPRGTQNSPFTSGTTPS INLPG LNSLTYYLVTVSSSEESTYSEPTTITQQTLIGDPGDINIALEDVDTNSVRITWVAINS ITRYTVGGGTGASQTVTVNSPEEAEY TFTNLVPGTSYTFFVTPLGTQRREREQYTRPDMPRNLLFNT ITTNS IS I GWEKPLTGGVDSYRVTYSPSSDGSPASPHTITDNG QITQTLTVTNLLPGTTYTFNVYALVAELVSEPVTRGSG (5) TTIGMDIGAGYTSTOFTVVWNVPTSGDFNQFQVTYDPYDPTNA QESPRI ISLSSLNGAQEGSLLIYGLEPGQLYNVYVQTMLNGLQTDDGFGRISFRTP (4) PLPVTGVVITQRTPYSLSLDWSDVI SADIQYRITYAPLEGALF PNNEDSAQVLSSNQVSESQISARSLAPGKEYVFT I S PVSGDPSSFEQVGEPETVVTYTQ (3) PLSP QNLVVSDVGVEEVSLSWNLPAEVNFPIAYYEIDLVPNEPTFPIRINGTRDKWE I HDGLVSGGEYQAVMRSVINDVNGQVIYSEP LQSAFFLLEPTQPQRLSTPYVTTTELRVEWTLGNSNFTTFQVSFSKDSATILTTDTTEQSLLLTNLTVATVYDISIRTVVEGPQ TNMIKTSAAALIMAVTTKSKPPQNLMQDEVTSDSITVSWGAPLNVVNMYHI IVKDEDGADVADATVPSSETSYTIENLNPYTKY I ITVAARFNGLDSDAIEIEQTTFQGK (2) PSVVRDPVVAINS PNSVFLTWTPPDSPNGILTGFVVEITGEDLTSRKRREAGFTR TETYGVTVFSATIENLPTGCSYTFS IKAANSADQSDAVIAGPLDLPHSA (1) PPTPPADRVVRLVSHTGTTMSVSFDSSIFSDV YGPVTRYALLVAQSSPDTIPSTPSDPAINWQVGSGQPYQTTLLTYAPFTNGNTRGFYTVGANNSCTQDSVGYCNGPVVPLTDYR FALRAYGSDGKFTDSAWS PLYNSG INTTWYAAAVVS I I I ITLVLLLVMLL ISRQCCGEARSSALYERGNGQHLPLVNAAYPSAH NQDSMHKT P L PEDAFIRMGP I PVRENAYVSMRDDRPKAPL PATLPRSAP PLTNPVQMRKFPDHVNRMSAGNNTGFVEEYNSLSN VGRDKSTDASRLAVNATKNRYRNILPFDETAVHLODLEEGOTSDYINANYIOGLHSMKEYIASOGPVPDSVNDFWEMVFENKST TIAMITGLVEGGKTKCEHYWPDDDTPVNYGSVTVTMTHTQEMEQWTVRSFLLEKGMQQFETHQYAFKGWVDHDVPNNARPMIEF IRTIDVAHDANLGPITVHCSAGIGRTGVF IALHKLIKQVETSKPNDY IDVCGTVARMREQRFNMVQTVKQYMF IHQCLLLLMR

>SeaUrchin4 SPU_016937.1 XP 783483. LOC578203, partial [Strongylocentrotus

purpuratus ]

MVVLLPYLPLVLIAGARILSVVNTANALTFLWDEQPNPETFTGYLITSASINEVI PKQSEETSYQKS ISNLTPGQLYEVSLFLQ PITTFPAPLDQLQVRTAPS P PES LRVVDETITTLRIEWDS PAS GLYRGFEVNISP PTALQELPRIVGSDIRGFTFVGLMHNTEY TVSVTT IMGEEGLEERSTTAEKTVMTA (23)RGDGDEVYLLSVSSTDAQI GWT I PPPTTVYLDGKQ ITVLSQPHTIPSLTPGRQ YTFTVEDVDLAFTTV (22) PERPDGLELESVGPYS LDWKVDTS LNDLNYERLEVSYS PES SQTDS PFDI SRCTDGTFTLEGLQP DTEYSVSVTAISGDTRSDVLTRYGFTT (21) CALEGQLHVVS LSSDEIALAWGPVNNI QGYAVRLFNAIGINMRTLSLSANSMS ATLDSLSPASNYSVQLIVMDTDGSRQTSVLQFVTD (20) PAP PGP ITLQSSTQTRLSFTWTPATSNFDSYELS ISGGDMPEPRP VGVVYPNEGNEFEVGGLLPDTTYNVRIYSVFFESRS IASEASFNTDEQ I LEFNVGSNQNLTVSWQVQEGAESYRLIYTTTNRNT NS IARPATSTSITIDNLLFGA I YNFRLATIAADGTEMEVANADFVOSPLTPSTNISDIKATTALLTFSLREGIFDDYVVMLSSP TDSCGARQPVFTLSREACPELLLLDLYPNTMYTAQVFARTGERGLSLPSETTL (19) QNFTTATVVNNEVEISKVGTNSLEI IW KTDNVDEYLLHYSGPDGPLMSDIVPY IE INYYTFGE LT PGTQYS ISVIS LINRERQGDALEVMOYTK (18) PDP I SNLVLTPQE NTINLTWT SP STMYDGF LICWYSATTEDNKAWLDGDATTYPWVISTPNEEYVVSVATFVGDREGDYVLSNKVTDFITLT (17) E TSPLILKDIATDT I SVSWVT I PGVVDYTLSYS P P GNEQATQDI DES SA IAEGLNNIYYVNFTGLTPGREYTFTVVWPLAFNYS TDAIRTN (16) PLPIYDPSVTNTTSCNLLYQWCLPDSDF DN IS IT IVP DVSMRTLSRGNGTGGMCDYQTVYLDGLEPATSYNVS VEVMS GDKSSEVVMVYSTTE (15) PLPVNNVTLDDST I FTLT ISWENP I GGS FNMF LVEYAPVRS PDSVMALDP INMGTNTA I I 
PNLQPGTLYNIMVYALAGEERSDATEITAMTR (14) QQNSEELRLQTRTSTS ISVSWGNTTRPEVTGFVLRITFQEGDAIDS IK NVSLGDVLGVEFSDLI PGTNYTVYLLVEVEGQLDTAVHSLDVTTL (13) PGMPGNI SCDPTKTTQSLLEIEWGSTQEGNFDGYA ISTQTGDGEE IPLESVEKDEQLQATADYLMDNT IYTF IVRSTLGDLRGEPTTMTCRTD (12) PQNLMVSTSTSPSGGQEVFLDW QDYGASSYKVEYTPQEAPLPVVQVI IVLRRPTSFTIQITLPRRGRYRFLRLRVRRRSQGLDAGPDGDEFLIPTDACPEYTFNNL LPDTDYAVDVNTVAEDGRTESSPSTMDVTT PRMEEGDISFNEVTTTSISFAWGMVSADTNGP FDFYLLNLKNELSI DVQNVQVN TSETREHTF I GLMPGELYRVELIVNSNRQRSAPMYTR (11) PDKPTDIMLSDLTTTSLTIDWKAPLTSYQGFRLCWAYGETNSI ELGPAVMERTLSDLEAGAEYLVSLAAVVGTGANQ ITSEKFTTIVTLF(10) PPAELHVNITSFNTTAVS IVWGTDI PLLDVSSY VVSYWETANNGSVQDISVDDPSQTDYTIDGLVPGTRYSFMVDALGTVQAVTSIGATQYTN (9) PETVAEIRVIPSITCSVRLEW DRPEGNLDSYIFHYNVTRGT IVGAPLQLDVAINDTAETLTYLLEGLEKDSS S DFVS LEVGVTTVAGAGEDQVESLQQVVKDLDV NVVT ITILGRDQNS IQAEVNSSVSNVES ITTGLYNAEMVTSSTTRNCS TMDCES FTVDFDTLNSGTLYTLYAAVVQS S GREVVP LLAKAATIPESAVDLQFTS I GRDYVVLTWDNPAGMI DSYNI SYYPVNDITKLMFEVVQAAAESNVLRVDDLNEGMNYSFTVVSI LEVEADLQEMGAPVEVFAV(8)

QYTIGVQPGIYAALEKTQSTV (7) PNPPTNLRTTMVAAQS ITVSWDPP SEGGSDTYNITYNVTGGQPPS PHTVSTSETSVTFSN LTPLTEYFFTIVNIWKSLPSRSELTRAT (6) TSAQTLSLAVTPEDNTEFTVSWEDLDTEAFEQYCVMYEPYEAQENRQQESFPV ARNIRSARFYGLDPGQEYTVYVTSCSSVSDPTSHANERISFRLD (5) PAAIAEIRIMDGS LGPRAVMLEWDQPEGIRGLYEVTL

DPADEGSVDVDGDELPVNITGVMTS IKILNLAAGKGYTVGITAISGDKRSENTTFLFYTL (4)

$\mathrm{K}(3)$ PASPS

DISVSNNELTRVTVTWQPSEGVVARYSYTYGLPGSTVTASETTTTTDTS IQLSNLTPSTRYEFRLWAESDQTEGSETSSSDVVT VNIVTT (2) VFFNVPSDPVSALPVTTLPSMPSAVGGLTITKTGIVDAEVSWSS PLLPNGVIANYSLTI H SHDNGTLSPYGDTV TISAVAEQTEYSQDVIGLAAGQYYVFSVYATNQVASSNQRSTTRTLFAETA(1) PPSPPRGATPSLVSRGSTITITFFNLFDTA FGRIVRFAI IVQERVDGGTVVITAKRQTTSS LTWAEARRTRPVPSYQTTPDDYNPFADGAGVTTFRVGSQSCDPDDLTAYCNGA LYPGTSYRFAIRAYGVDGKFVDTEFSSPFRTDPDRGI

NGNGYSNGNGNG IMKRAL INDGVNTYAYQQPQ IMEHSFTN I PDYST I P PNRGTTAQVHQP S TTHTRPTKKNMFAQHVAKMSARN NAGFSDEYNNL PAMEKTRAVTAARQARNDDKNRYRNILPYDYTRVQLKGVAGTDYINASYIKDDHGQKKY IATQGPLPNT IEHF WEMVWENQTSTIVMMTALVEGGKTKCEHYWPAGEEPQLHGNVTVTLVGSNQTDNFIERTLLLEKEGTERTVTHYQYLAWTDHGV PESTAPLVGLLRQVKVTNQEDGAATGPIVVHCSAGVGRTGTFIAADMLMDAIQRSTATDY IDVAGTIAKIREQRALLVQTLYQY IYLHRTVLSLIEEQ.

\section{Hemichordates}

>AcornWorm gi|291235644|ref|XP_002737754.1| [Saccoglossus kowalevskii] MGTQDDTLTGRVLIIIQVFIFIGFSNAGYKCTDDCAYSHDNLCDDGGVDSYSNYCYLGSDCDDCGPREVPDTTTVAPPTTPTTP TTPSSTTRTTAMHAMQTTFVHTTTAASEAEHAHTTLNTSTDASKTTDLQTEGHETSGAESTHTPEIVTDVIHSSRTEYEVMSMM TTNENEENNVTMYNIRTEEQETEVVDSSTLVGTEIVITGFTPTEEQETEVVDSSTLVGTE IVTTDFTPTEEQETEVVDSS ILVG TEIVTTDFTPTEEQETEVVDSSTVVGTERVTTDFTPTEEQETEAVGSSTLVGTEIVI ISFTPTEEQETEVVDSSKLVGTEIVTT DFTPTEEQETECVDSSTLVSTEIATNGFKPTEEQETEVVDSSTLIGTETMTTDFTPTEEQETEVIDSSTVVGTEIVI ISFTPTE EQETEVVDKTLTTDFTPTEEQETEVVDSSTVVGTEIVTTDFTPTEELETEVVESSTLVGTEIVTTDFTPTEEQETEVVDSSTLV GTETLTTGFTPTEERETEVVGSSTLVGTEIVTTDFTPTEKQETEFVGSSTLVGTETMTTDFTPTEEQETESVGSSVVVGTDVGS TGGAQTEGPETEVVDISTVVHTEMVTTGGTPTEEQETEVVGSSTLVGTEIVTTGFAPTEEQETEVVDSSTVVGTEIVTTDFTPT EELETGVVDS STVVGTEILTTDFTPTEKQETEFVGSSTLVGTETMTTDFTPTEEQETEFVGSSTLAGTEVTTTGFTPTEEQETE SVGSSVVVGTDVGSTGGAQTEGPETEVVDISTVVHTEMVTTGGTPTEEQETEVVGSSTVMGTGVMTTGVTQTEKQETEIVGSSK VDGTEVVTSGLMPTVEDGTGVIDSSRFASTVKVATRSTLHSTTMVRPTEEDTESTTVVLSTLPTILAVYVTSTEVQMADDSYLR EWSDSVALTFDLKLSTNESLQSVS LNNDWKLI IY IRDVSSTNETVNERLRPYEVEVDNFQ I SGS DLVFNDI IVNMNLSGLLCSE MTYICIELIAYDGNGTDLVF IREPVDCVS I LCRGVEIAPFVDVVTGSKLREGMDDQSFQFNLILYPDPEAGS IEGEYLWTLTSF FSQSNDGYGNTDAGTVHSLTTDQSGMSLYSGIPASWTGFRSSAINITDKLCSDVSKYFCVNISKSSLSNPDFTFGNPVMTCTPT PCTGVDVVDTRLSIVSGIVVKEHESSQYFNVFVTLLSSPDFGDIEGASLWNVTMYVNTQGNANVGDVSALAVADI PRDMRDLSL LAGSNAILEGVEAFMDLAELNCRQ I RYLCVDVSRDQTSVPAFTLTGKLQTCAEITCRGVEITDTELIVKSGTS IVERSGNHSVT FDVLLSSNPAAAS ISGDRLWEMYVYLSDVEFDDTHSFAATYPVLSSVEDTDLTAGVTLALLGIEAFLDIDSLTCDQMRYICVTV FKGRYSSPEYTLTGVPDQNILTACQPVTCTGVI ISTDLQLSDDFYLLEGVDNQHISYTMTLFASSAGAGVSGMNLWDIRTQLRS IEDA IVDTSNGTIQEVNTTLNAGGVLLMSNVKSVLSLEGVLCHQFFNICAI LNKNSNANTEYTIEYDRSTLVSCRSVSCKGVEI IEVAPT IMNGDIVAEADDAHAVS INVGMT S S IYGTNRTGAGSWALEVFVTSDDVRMPKLVNLYATLTAAQQDTELIGGIPSVLS NIEVELDVSALQCSEFTQLCVVLHKGDNAEPDFTLGVLQDENALPGCAHLSCQRVAFNISAINVTTSSFT IRWDEAVGDFDGYY IDVSPALTGLSSSFITERDEPREASFFDLESGQEYVISVGIAKNGFPKSTGVTQYTQRTF (6) PEKVDSLRVSMVTETEI HVVW DRPDGVVSTYVISIQPSEGTATLPITLPSDSTTASFSGLSPMSQYVITIVTYAGSLQSKEVPVKVITG (5) TIPPVLEVTSFTM DTITVATYVDNRLSHGMLHLSYFPTDAEP I I ELDLNKTSYVITDLVPGRLYTLEVKIVADIDGGRITSNSDRKYQQTE (4)PS PPGS IVVSRITEFGAIVNWEPSSGDFNFYLVTYSSASVNTALPIRVNHGQTPQLSLSGLSPATVYTVHVSTRSGELTSQSVSTT FNTL (3) LAPPGRLSVNGVTS SSVQISWLPAI SGLAVEYYVTYAPFVEGATPASPFTLNGTSVNITNLYPFT IYTVTVQTRIDD MYSRERGITATTYPDL (2) PSSVKGFNVTVES PYQVTLTIQQPSFANG I LESY IVNVVGYKEGFPPHILDFE I P PDETDDITEY LLEDVIAGYTYTFTVKPQNSLGIGPLRTAGQVIMPIYP (1) PPKPTASRITANVRFVETTAYTLRIHLSNNLFDDRFGI IVAYI VI IAEDGEYSPLPEVLPSYDEVIDSSPWP PYQTSQP FNPFETLS I GRRRRNTLQTVNYI I GAKENCDVSLVYCNGALRPVTDYR YVIRGYNELGNYTDTDWSLPQKTDLDPFWILY

FNGNEPTLEKTEKLYTLSLRRKRKSRPVPLKGFRDYVNELDEDDRKGFEEEYDDLRKLVVRQPTSVGQRMEHRSKNRYTNIVPY DNNRVILSGSGNSY INASYISGSKGSRSYIATQGPLESTCGDFWKMIWQQRVTTVVMMTQCNELGKSKCHHYWPRDTQTQISHS DLTIRLTSETRLPDWI IRDFS IESNGEIRALRQFHFTSWPITGTPYDADPLIRFIEAIRIQVLPNSGPILVHCSAGVGRTGVFI ALYHLLEYFYTMIQVDIFGKVIKMRKQRPFMVQTQGQYEFLYYAIQQHIKEKP LGTNWSMRKTMDSNWT I TYGGTVDPDDSGPG FFDPYDPEEV 
>Acorworm1. XP 006823994 curated profilingl partial [Saccoglossus kowalevskii] MS LKHAFCFPANVNV̄IDYGTDYITVNWTHSGE HVDEFVITAIATENEAANGTETVNITRGTVDGLTPGAEYTITVVAVSQDDVE SLPSDEVIQRTIPEPPYNVTVESYNTDS I HLRWEPPLSNVFTHYNVTYVADSDPGMTVTVNKSTTESSLTGLISGETYNITIKT ISGDESSGASDAVVQTTE (16) PNSVVLARDETQITTDTIAVTWSSYSGDIVDTYTVQCS SNGTAEEATVDYVNGTDTYSAKCT GYETAGKLENITVTAVSGTSGPSQSLSEPSMIEIHTK (15) PNSVVLARNDSQ ITNDTIAVIWNSNAGDEVSYYTVECSDGGTP GQAMI I YDNTVTYSAKCTGYGTAGKLENITVTAVSGTSKP SQSESEPSKLAIHTK(14) PKSVTLARDETQITNDTIAVTWSSY SGDIVNTYTVQCSSGGTAEEATVDYVNDTVTYSAKCTGYGTAGKLENITVTAVSGDINTTGSESEPSTMDIHTK (13) PNSVTI ARDDSQITTDTIAVTWSSNSGDIVDTYTVQCSSGGTAEEATVDYVNDTVTYSAKCTGYGTAGKLESITVTAVSGDLNTTGSKSE PSTMEINT I (12) PNQADPVRNDNLVTNTTIGVKWTDPGGN INHYEIGCPDGGTPSANVTHTPDKEMTAQCTALATPGGLYSIT VTSVSGNSKSDPAVTSMNTK (11) PNKVRLERDDDNITNDTIAVTWNSNSGDEVTYYTVECS SGGTAEDSRVDYVNGTDTYSAK CTGYETAGKLETITVTAVSGDLSTTGSESEPSMMEIHTE (10) SNAITLTKDSEYVTTTS IAVTWTNPGGAVAYYTVECSEGGT AENGS I PY I DNDQHEYS GKC INYNNP GNLETINVTS INADNHRGETSSME INTE (9) P LPVTDLTKVDATTAF IEFSWIEPESG TWSGYDVGYLSEDGTQQFSEYLSDDVHTITSHTFNNLTAGVLYDITVITVSGEEMSTNETEPMRTI (8) PNAVTDLVLTTPNQT NPEIVIMWS PVEDEVSHYRVKCNDSDDVTTEPDDQI I PHENVTSLTTECSDLFPGRLYNVSVSS I SGYEGVDETESEPIVKAKR TH (7) PKPVTS LS LTS P DNSVDT I HVSWTAPEDSDVDGYRVVCTETDSGDKI DDVEVTVTNYDF S GLTAGRLYTIDVYSYSGSD NDEKLYSVNTTDVKRTY (6) PKPVTSLSLTSPDNSVDTI HVSWTAPEDSDVDGYRVVCTETDSGDKIDDVEVTVTNYDFSGLTA GRLYTI DVYSYSGSDNDEKLYSVNTTDVKRTY (5) PNSVTITSTSTTTDS LNVTWS LPDGNWTGYRIEYVPODNLANAVLRRED ISDPEQKIVHHTIYDLYAGTLYNVTIFTVSSGIDQLESLPDTTEDRTV (4) PATAYNVTEVESSKTSYSLEFSWKINTTSLHDY YEVYYRGQLDTSFRNTTSDSENVSLVDLRAGDTYNVTI ITISGKKRSAPSEPAYGTTT (3) PLSPGAVSVIDVTETEIKIQWAQ ACENCTYDYYVHHEPEDGEVDVDSSS LHAKLYDLNHGTLYNISVVS I SNDVPSEPTYTLARTL (2) PDTPENITIETGMRS LNV TWDEPEGEYAGYNISIEPSTNRAGKDI IVDVPDKGIEECI ITECDPYTNYKITI IVHSDEPNQTFSEPAVSHAMTETAP (1) PP PPRNNIVPSSSESDIEVSQTTITVLFTDDY FNDSNGPLLNFTVIVTEDGTEEVDKHLNGE I PRVYCKDCENVPGIEEGEVKTWA EVQNTPARYQIDYPYDYPDLSRRKRDTTGIEITIGSDDSCSTKDEGFCNGPLKDGTSYRYHFRAYTDVGYSDTVLSGPIRTSTN MSWLW YKRRYYEETTTRTVEGTANPEFIGLRDENGKPPIAQKPKTQKHSKPVKLAEYHGYFOI MSADMEFRFSEEYDELRPVGREQSWDAAELPENRAKNRYTNILPYDRTRVKLSQVEDDEATDYLNANWMPGFNSPREFIAVQGT LPGTKDDFWRMIWEYNVSTIVMVTQCQERGRVKCERYWPTDDNPVYYGDVLVTVTHENELTDWVIREFTVENGKSLRRIRHFNF TAWPDHGVPEETGSLIKFVRSVRAQIQNDGTPTVVHCSAGVGRTGTF IALDRLIQHIKEYDYVDIFGIACEMRMHRVYMIQTES QYIFIHLCVDDLLKEREQPDEPDEPDSTPDTTEH IY GNVPSVTVMRNGPDVVTANLGDAYKRNSVIAQA

\section{PROTOSTOMES}

>Nematode. 2 CeNTP10D C. elegans. F44G4.8a DEP-1 NP_001254192.1 [Caenorhabditis elegans] MIRWKYELHSLIWLFLVLHLSKCQSDSLTTSAEQHELFAIKKDSLSPWSQI LVSLPRRHPLYQSFAAKIQDVTEN ISDEVRDSN KTFVSSDDAPYS IRIHALRAGHRYS IAI HGQKDGSTSLIKEESVVMDPRAPDFRSMDSDIQVAEHNITMRTIKNDSYLQDSFS I EYRQINPDKKFPVLQILDIPEQKNLEFYLGNLNSGFDYSVRVIAHKDGMSSRPWISTLTTKPSPLKEVNINQNAGSCVEVSWQN DEFSGADFYTIQYSLQSTPNNSTNMTIPSTESS IS ICDSMLQGEAYQI IATVQKGGQVSEPLITKFQLRPLPP I DFRVRADLKR GKYKLLAELPTSSKIDKCQITVAGDEAERSVNYANIEQTKSGHKICWFNFALSPGERFDFS ISSMANESASQKLQKS IVLTPAF DFNAFGLTLQESNGGIELIWPKSEVFMTRVKDIWNKVVGAESLLNMRITPIGNNDETDKTLKFETSPKNIDPVFAKNLVKGACY RVQLFTVTKTGI ISETRHNET IRMS SPAVNVSLESVTRSSATLRIVFSTHHDSTS I SNCQMHIVVRDMNGKSVFDKRMQLTATF APLLNLDGLSPFHKYTVNTQI ICGSGSSETPQCPAATRTMRQLSFSTRQDKPAAVQDLKVEPLNSYSVMLTWLPPALPNGILTH YAVNVTKIGSDETRTI DVGVSSNRSDHTVQVVIDELFGGHTYSFSVRAVTEAGFGENSPVVPTVSMPLMAPPVPTVAPMIMKES VGSHNMIVRFPTTMFDNRNGE I KQFA I IVSETTADES INRW I ES DNGTYTWQQVQRFDVWP SYVAKLQD IQKVKQDVDVS I FEE LGEDETCLEVRADRICNGPLRSASKYRVRIRLFTSPTLFTDSP P SVMTTGSATPAIPLLTVVAVLIVIAFVGIVGTIFLFFWN RTKKARLAAAAFKNGPSKEKESQWEALKMMMAERAADCLAKLGLDATTP PPSSTTSSNSPTSTSTTMTDCGSNPHLGAPNAGGH RRTRS LRERTGVEHRLERLSS GPVHRTPLYTVVTGANTNKSRPVRI EDFADHVRLMSADSDFRF SEEYDMMRNVGVGOSVAASE LPINRPKNRFTNI PSYDHSRVKLSNPNNIEGGDY INANYVPGFSSRREF IAAQGPLPTTRDHFWQMTWEQQCPAI IALTKCVEK GRDKCHQYWPDHENVPVLYGDIEVTIVAEKEFDEFVIRDIRLEKSGPDGRVTRFVRHWHYMAWPDFGAPSHPNGIIQFSRMFRH HLPHSPHNAPTIVHCSAGVGRSGTFIS IDRLLOSSSFGDPIDVFGTVCEMRYERCOMVONEOOYIFIHYCILOVLOGS S PS PTS TSTGAHHNAGFVQDGQMIVESGF

\footnotetext{
>Nematode.3 HCNTP10D Barber.Pole.Worm gi|560121775|emb|CDJ93597.1| [Haemonchus contortus]

MVGLDVALEVVMVIIRTVLTLATAIVFATKELRAASNAESSEDTHEKKLDLLEFKIEKDOKLPDSOLTVKLPYNHPSYDQF IAK VVDISSTIDPS IRDVNRTFLSSADGSGTIRI HTLHPGHKYSVSIVGRKGENSSVIKEELVVMDPRVPEFTTQNSDITMHNITLR AVKNEKFLEDSFLIEYRQLEPEQGY PVLEVLDI PEQKNLEVYLGNLNPGRDYSVQVVAVKTGLKSRPWSTTLSTKPGAVSDLIV SENSSCLTVEWDVP PNSGADSFLLRYRQLNAAVNLSVTIPGNDRFVDLCDGIVPGTAYALGVVAKKGKSVSDETIKTYTVRPAS PTDFOVSPDITKGKYRLTFDLPPNSNYDGCHVSVVSETLEALEDDGEVNEKEGNKSCS ILMPLVPGERFEFTLSTYVENVTSTK LHRSVVLTPAFDMTGFGLSLQEYQSGVKLSWPQSDVFMSRMRDIWNKVVGPKSQLQMRLFPADGLEKGSRLQGDPHEATPLIVG ALKKGSCYKVQ I FTVTKSGIVSEKRYNDFFRMSAPLVNITVKS ITRSSAVI HAAFVSP GEADPECLLNA IVLDMHSHVVLDKTL KAQSHNFS P IELGGLRP FHKYTVNSKITCASGPSDC ISTSRT IRPMTFSTMODKPGPVLSLSARPLNPYSAQLMWLPPALPNGI LTHYVVDVKSEDDPNDSRS LNVAIGTDRADHFVETVIDGLSGGERYTFTVRAVTEAGTGELPTALLEPVRMPILAPPRTSTVPI VIAES ITS HS LTVKYS P TMFNNKHGRI IRS TLLVAEVTEFGQ ISETWMNAENETYTWMQVQRFDVWPLYAAAVDEISARGS PSI SQAVGIDQTCEDLPSDVICNGPLKAGTNYKFKLRLFTATNLFSDTDFSEIITTEAAARGA
} 
YWNRSKKARAAYASSKESQWAALKMIMAERAADCLAKLGLDGSMHNGE I PSQPSHLQPTFGHHRRCRSLRERTGVDHRLERLPS GP INKTPLYTVITGVNTNKSRPVKIADFAEHVRMMSADSDFRFSEEYEILRNVGCGQSYTAAELPVNKPKNRFTNILPYDHSRV RLMQAGDQEGADY INANEMPGYSSRREFIAAQGPLPTTRDAFWQMAWEQYCPAIIALTKCVEKGRDKCHQYWPDSEHSSVVYSD IEVTLLNESSYDDFTIRELRLKKLHESTAPRI IWHFHYMAWPDFGVPDHPQGI IRFAIMFRSRLPHSALNKPTIVHCSAGVGRS GTFIAIDRLLQT IQADRPIDVFGIVHEMRYERCHMVQNEQQYIFIHHCILHAIES IASERTGMT TELHQNPVFEDDDT IAE SDF

>Annelid gi|443734862|gb|ELU18718.1| CAPTEDRAFT_229306 [Capitella teleta] MSQPVTNQLAGFGVLAALALICVSRSESSSTTTAAVPTTNFSTNIPPTTQGLEVTEVTTSMVTLVWSYDGSEQLTFVVEYVEKD ADNATSFVLEQGVTSCVVEGLQAGWTYTFEVSVQLERDMLGEPAYANATTIPGKPINFRTVDNSVGATFFTLTWESAAGSQQDD FVVVYRKIGSSDVVQVFTGGATSVTLHGLDAGVLYEVKVMAVRGQQLSTPAIITEVTR (12)PPTPRIHNITAVDTKSVTVQYS AGAGGSQNSFVVTYVNQEGQTNEPVCSYSNTCTFDVSVFGVKPGEHFTVKIKATQDMVSSAWASMSGNTKPLPPSAPKLFERSE TTLTVIGDVPSTNSTVFDGCKASVTPAIQNGPYVFKRNGGYEIRLQNPTPGSKYAINIWTYSREEESAHVSGDFYSMPAQPGVI

(11) PESASNLELKTTTSSTLVVQWDEPSTGFYDEFLVRIQSHGETEKITKS SQSTYEKEFDNLSAGTEFTIVVITVSHG QQSTATIGIFYTK (10) PERPSNLKKESTTSDS ITVKWNAPVSANF DSYRITINSTDHENEVLDSSES SHTFS GLMPAQQYDIT LISLLNSLESSMLS IQVYTN (9) PAAPTDLRI LSRNTNMMS FTWS P PTNPGT I DDYKYSITISGAATPE IERSGTINEYSHQLS AGKTY I IEVRAVTNNQRGLPVSLTFTMI (8) PNDPGNVREMERGQSYLKIEFDAPWDTYDGFEVTWVEKGSGPSSVADLLVTSY NDDS ITVEWEKPTGTTVNGFTIS I DPPPSGQSGSKDLTENIQEYTWTGLSAGREYNLI IEVIEADTKSEKVERRQTTK (7) PKR ALSLSANYVSTDS ISLSWKVDQSSSQDNFKITYEVTGSSTEWTRETTFTQGQLQYSYLIEGLSAGSTYS ISVIAKKSSVQSDPI EITQTI (6) QPKSVRELKAEVIPSG ISLSWLPGFDSTQNSYRYQYQGKNVKLNIVPWVVINSESVDLQNLFPGERYQFYVDAIS NDQYSPSNQSTLATTY (5) PLPPTDLTVDRSATTVS SVRVQWKDDVTRSYITSWDIKIADRGTDNIRRVGSTHERTNLNYEI PN LTAGKNYTVYVYGKSGNQHSRDASTMDVTAK (4) PVIRSTLSEDTEKTTEDTIAVTYSESKAGVFDHYLFSLLNSSDTVIKQRS NTDRNIRFENLVAGVKYTILARTVSGVEESMTTQKSITTK (3) PNKPKHKCNQGTDKLTI ILIKPSGFVDEY ILKCLNEDCNST EISNVSFEQDFNNLKPYTTYHFEAYTKRFEKLSNKVEFPCKTGEGP (2) PGPVQEFSYSENSLKPFEIRLTWMEPRNPNGKI IK YHIKYNGIKENQVPHTGSEVDVEPDMHEQLIKGLKPGFEYTFEIAAETNAIGKKTTLVKTMPIKA (1) P IVMPNSPPPQA ITTA I SHDKIRI I LTNP F LNTNGDVVAFSVFVTTDPNERFMANS PLRTWADVKGP S PMASYFAVYKCANLFDGNDQC SSGPARKRRVA QPRNTVEFTVGGDSSCTTNADDYCNGPLDAESTYYVALVGYTENDLYSSGPSSEPIRTDTAPTNLLI

GVIVYMRKRSS DNNEDKLVCCLVLIGVKPASPEWPP GSNNGMVI I PS SKPVKKVDFVQHVASMAADSEFKYSEEYEELSLVGKD QPKEASDLPCNRSKNRFINILPYDHSRVKLLPIDDEDGGDY INANWI PGYNSRREYIASQGPLPSTRDDHWRMIWECNCQCIVM LTKCVEAARQRCDHYWPIDSEAIYYGDLQVQILNETKSTDWSISEFI ISKGDQSRRLKHFHYHSWPDMKAPNKTGS IVPFVRLI RAKVQLDGGPLLVHCSAGVGRTGTFIALDHLMQHIEEHDDIDIYGTVYQMRKHRCMMVQTESQYIFIHKCLEFVLEGGEDDEYA NTGA IGTENRAFEGLRSESDLVAEIFFNLPRQALTNFDDDEGIDVKSDP

$>$ Fruit Fly DPTP4e L20894 FBtr0301793 Drosophila melanogaster

MDCATRKQQQLRAHHQQQQI $Q$ I QTHGRKRQQLQKQRHNHHHYYQNSQQQQKHFVWLVVGILTIFLAQHANAADLVINVPNASSN ANAFYRIDYSPPFGFPEPNTTIPASDIGKDIKFSRALPGTEYNFWLYYTNSTHREQLTWTVNITTAPDPPANLSVQLRSSKSAF ITWRPPGSGRYSGFRIRVLGLTDLPFERSYSLEGNETLQLSAKELTPGGSYQVQAYSVYQGKESVAYTSRNFTTK (1) PNTPGK FIVWFRNETTLLVLWQPPFPAGIYTHYRVSITPDDAIQSVLYVEREGEPPGPAQAAFKGLVPGREYNISVQTVSEDETSSVPTT ARYLTVPERVLNVTFDEAYTTSSSFRVRWEPPRTYSEFDAYQVMLSTSRRI FNVPRAANGDSVYFDYPDILEPGRTYEVVVKTI ADNVNSWPASGEVTLRPRPVRSLGGFLDDRSNALHISWEPAETGRQDSYRISYHEQTNASEVPAPFPVAAESQITTNLTEYTLD SLLAGRRYLIAVQALSKGVASNAS DITRYTRPAAPLIQELRS IDQGLMLSWRS DVNSRQDRYEVHYQRNGTREERTMATNETSL TI HYLHPGSGYEVKVHA ISHGVRSEPHSYFQAVFPKP PQNLTLQTVHTNLVVLHWQAPEGSDFSEYVVRYRTDASPWQRI SGLH ENEARIKDMHYGERYLVQVNTVSFGVES PHPLELNVTMPPQPVSNVVPLVDSRNLTLEWPRPDGHVDFYTLKWWPTDEEDRVEF KNVTQLEDLSSPSVRI PIEDLSPGRQYRFEVQASSNGIRSGTTHLSTRTMPLIQSDVF IANAGHEQGQDETITLSYTPTPADST RFDIYRFSMGDPTIKDKEKLANDTERKLSFSGLTPGKLYNVTVWTVSGGVAS LPVQRLYRLHPLPISDLKA IQVAAREITLHWT APAGEYTDFELQYLSADEEAPQLLQNVTKNTEITLQGLRPYHNYTFTVVVRSGSIQGTDFADVSVSTLMRSSAPISASYQTLTA PPGKVDYFQPSDVQPGEVTFEWSLEPAEQHGP I DYFRITCQNADDAADVSSYEFPVNATQGKIDGLVPGNHYIFRIQAKSALGY GAEREHIQTMP I LAP PVPEPSVTPLEVSRTSSTIEISFRQGYFSNAHGMVRSYTI I IAEDVGKNASGLEMP SWQDVQAYTVWLP YQAIEPYNPFLTSNGSRKSSLEAEHFTIGTANCDKHQAGYCNGPLRAGTTYRIKIRAFTDEDKFTDTVYSSPITTERSDTVIVA ATVSAVLLVAMVLVVVYCQHRCQLI RRASKLARMQDELAAL PEGY I TPNRPVHVKDFSEHYRIMSADSDFRFSEEFEELKHVGR DQACSFANLPCNRPKNRFTNILPYDHSRFKLQPVDDDDGSDYINANYMPGHNSPREF IVTQGPLHSTREEFWRMCWESNSRAIV MLTRCFEKGREKCDQYWPVDRVAMFYGDIKVQLI IDTHYHDWS ISEFMVSRNCESRIMRHFHFTTWPDFGVPEPPQSLVRFVRA FRDVIGTDMRPI IVHCSAGVGRSGTF IALDRILQHIHKSDYVDIFGIVFAMRKERVFMVQTEQQYVCIHQCLLAVLEGKEHLLA DSLELHANDGYEVTK IYLERQPQTKMGTLPIRASLAMAEKLDADLMTNKDEDEDQEQQQQQQLQLATEVKPKGSNDDEEDEEDD DDDDDDQQPLNNETTATLSSASCSSSTHDVHVVLQEAIEKPKQEQERICAGTQSHADTES DNTDS DDDDEDGDGKVAKDGAVAD EDGWWY

>Fruit Fly PTP10D M80465 FBpp0303419 Drosophila melanogaster

MLYQLSKATTRIRLKRQKAVPQHRWLWSLAFLAAFTLKDVRCADLAIS I PNNPGLDDGASYRLDYSPPFGYPEPNTTIASREIG DEIQFSRALPGTKYNFWLYYTNFTHHDWLTWTVTITTAPDP PNLSVQVRSGKNAI I LWSPPTQGSYTAFKIKVLGLSEASSSY NRTFQVNDNTFQHSVKELTPGATYQVQAYTIYDGKESVAYTSRNFTTK (1) PNTPGKFIVWFRNETTLLVLWQPPYPAGIYTHY KVSIEPPDANDSVLYVEKEGEPPGPAQAAFKGLVPGRAYNISVQTMSEDEISLPTTAQYRTVPLRPLNVTFDRDFITSNSFRVI WEAPKGISEFDKYQVSVATTRRQSTVPRSNEPVAFF DFRDIAEPGKTFNVIVKTVSGKVTSWPATGDVTLRPLPVRNLRS INDD KTNTMI ITWEADPASTQDEYRIVYHELETFNGDTSTLTTDRTRFTLES LLPGRNYS LSVQAVSKKMESNETS I FVVTRPSSP I I EDLKS I RMGLNI SWKS DVNSKQEQYEVLYSRNGT SDLRTQKTKESRLVIKNLQPGAGYELKVFAVSHDLRSEPHAYFQAVYPNP PRNMTIETVRSNSVLVHWS P PESGEFTEYSIRYRTDSEQQWVRLPSVRSTEADITDMTKGEKYTIQVNTVSFGVESPVPQEVNT 
TVPPNPVSNI IQLVDSRNITLEWPKPEGRVESYILKWWPSDNPGRVQTKNVSENKSADDLSTVRVLIGELMPGVQYKFDIQTTS YGILSGITSLYPRTMPLIQSDVVVANGEKEDERDTITLSYTPTPQSSSKFDIYRFSLGDAEIRDKEKLANDTDRKVTFTGLVPG RLYNITVWTVSGGVASLPIQRQDRLYPEPITQLHATNITDTEISLRWDLPKGEYNDFDIAYLTADNLLAQNMTTRNEITISDLR PHRNYTFTVVVRSGTESSVLRSSSPLSASFTTNEAVPGRVERFHPTDVQPSEINEEWSLPSSEANGVIRQFS IAYTNINNLTDA GMQDFESEEAFGVIKNLKPGETYVEKIQAKTAIGFGPEREYRQTMPILAPPRPATQVVPTEVYRSSSTIQIRFRKNYFSDQNGQ VRMYTI IVAEDDAKNASGLEMP SWLDVQSYSVWLPYQA IDPYYPFENRSVEDFTIGTENCDNHKIGYCNGPLKSGTTYRVKVRA FTGADKETDTAYSFPIQTDQDNTSI

LLFYKRRRNNCRKTTKDSRANDNMSLPDSVIEQNRPILIKNF AEHYRLMSADSDFRFSEEFEELKHVGRDQPCTFADLPCNRPKNRFTNILPYDHSRFKLOPVDDDEGSDYINANYVPGHNSPREF IVTQGPLHSTRDDFWRMCWESNSRAIVMLTRCFEKGREKCDQYWPNDTVPVFYGDIKVQILNDSHYADWVMTEFMLCRGSEQRI LRHFHFTTWPDFGVPNPPQTLVRFVRAFRDRIGAEQRPIVVHCSAGVGRSGTFITLDRILQQINTSDYVDIFGIVYAMRKERVW MVQTEQQYICIHQCLLAVLEGKENIVGPAREMHDNEGYEGQQVQLDENGDVVAT IEGHLSHHDLQQAEAEAIDDENAAILHDDQ QPLTSSFTGHHTHMPPTTSMSSFGGGG

>Fruit Fly.Ptp52F-RB FBtr0289985 Drosophila melanogaster

MEATNWKQSHRRIHALILILWIAGVQGQTVGSLVAVSESQELAEKVEVTVVSTVYTLGFS ISNEADYS IDKVICRNGSGSETEA NAAHVCENLNPCTFYTSVVSFRSNVSGKPPPSDQTIYAYTEYKQ

L (3) VSAVYEPSPTAYPTQLHWTWFSPQNHPKCVANYSVTLTGPIQRSENKTMNVITEETFAIFDDLDPCG IYLVEIVPNQLNGSAGTKYQEQSTVGEDQPSVIQDPVVEAEAYSMEVSWKTPEYADLCIDGYRLSGWMEDDKLVEVEALS ITTQ NTTVVFDKNLLACQVYI IQI I PYTKENLDGQLRQVGVETKAAIVDYTKVKLEMKNAGSEFIDLIAFNADYNNSCPTIFALFTCN ATTQVRNPYAERYVEGHSKQGFNASLSPLSPYATYVCKVILYNVAGPSEPVVDADMHTTTYFPEQPESVMLEKSTVSSLLFNWQ PPTYTNGPIKYYQAFLMRHEASYFVPADCAIVEQDTKSETKGDPSVNFTGLAPAVRYMMQVAAQNDFGMGVYTEPVIGITLPAV (2) SDSVTQLTVLTQGPVNNNAVYEANVTITWKVPCKSNGDIEYFQLAFNGTRNNFAPVSFERRVELDTGNKQGRMSYTETEMQ PQFDYTVEVSVKNRDVEQLSSSVPGSWQSPAGL (1) PTIPSDELIKQMRANVEETSNPTKTAIVRLPADIMTSASGDIKWMALM ISQKNCAGVPHLKYDVSSDWPKVLSYQEAGADGTGDCSLEYQTTEERWHPEPVQRQRRDGEVTSDEEIVETIGLDKCSEVQKTY CNGPLLPDTDYNVVVRLFTASGYSDAAVLNFKTKAAIK ATQGPKPESVMDEWRMILQYNVRVIVQVTQFREGNTIKCHEYYPYNVRGLTVTIKSKEVLELYDRTELTVVHDKYGLKEKVIHY YFKKWPDHGVPEDPMHLIMF'VKKVKAEKRPSYSPIVVHCSAGVGRTGTFIGLDLIMQRLKSESKINIFETVKKLRFQRMKMVQT QQQYTFLYACTYELVKHKI PRAALKMDGRPKSVTVPAIPS PKKVSFPDVDVGSEYVS SAP ITDLDDGRP IVQLPSRFSGLRRNS PPGENDNPTSSSNM

\section{PORIFERA}

>Sponge XP 003383454.1 XP 011409595.1curated by ptotein profofiling [Amphimedon queenslandica]

MFFI IDILIKNFYLGFAPDVINFTVNSGNSTSLALSWQLRYPLVPPDMYYIAYNYTELSGANPRSDNST IMIDEDDVTTTTDYI LYTLDDLLPFTSYYVTLFPQYGEEEGEGLSDSGNTGEGMM (8) SSQVNDTNVDIDRDNMRLNISWAPPSRPAGNILRYEVTLSI RGKICLTINTTELSVIAEFKFNVTYTIRITPFNSFGSGTTRELDMFATPEGA (7) PTPPLNFTLTELSLSSLKASWEEPSTLNG VLANYTVYCNLSSLQFYSQQLMLS FNAPPTTVDPDSTTANITGLYPFTNYDCYATASTGGGESSRSNNDSARTSEEEPAGPPEG FNVTDVTATSVSLEWRRPSVPNGVI LHYFLQYSAYNVTIPVTFNTSSDEYSVTSYTVESLNEYTNYTFTISAVTSGGAGPLATT STRTSEAVPGTNLDGFNTSSMSLRITWTELLEDDQNGVI I GYNISYFSLPAVGQPINNFTSDTSYNISGLDVYTDYNVSVAAYT SAGTGPFDSVIRRTDSTV (6) PTSPSDVSYINISSTS IEVSWNPPTDFNGPNEGYVITYIRLESDTESMSTNRLTGTSEVIENL EKYEQYSVTVVAFTDKGPGASSDVLSVLTDEDL (5) PGPPSNVSTMSTNTSIS ITWSPPLDPNGLLLSYS INVTLNSTYAQYLS FDVMTMSVSQNIFSYTLFDLLPFAGYDISLQASTSVGLGTPAIETETTLQAA (4)

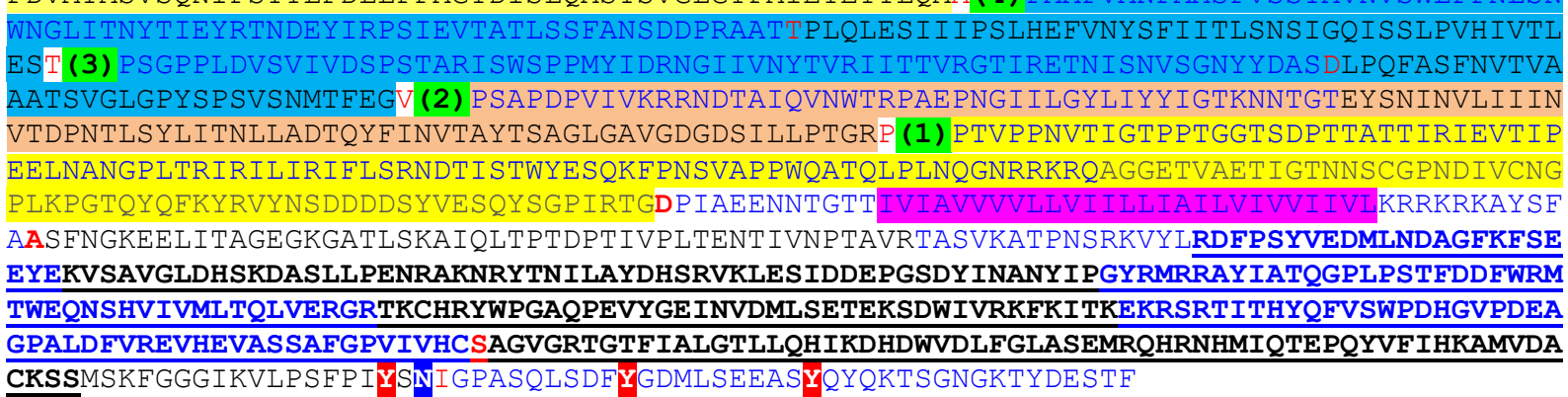




\section{PROTISTS}

>capsaspora PTPc1 Capsaspora owCzarzaki ATCC 30864 ]

PTPcapsaspora .2| EFW39745.GENScan ref|XP 004365141.1 |

MKPRLLLLAVALLAAVGSVRGADLDITFSAATGNS ITLNWVAYGSFAS PMEYRVTRTPGSATPVYIGTALTFADSSLTKNTAYT YTVSVYSNSGAGGTLVGATAAPPSQTTANDVTLNADS I LMYATSNVNEYVLSWPS LVANTGASTAGPPATITYTVEYKKVASGS Y IGWTGYSSGTSAPALVLAPGTNYNVRITVSSGILGTASVNQVFTGRTYSVAPVIGTALAAGTITATQVPLSWTVTSSGQESNL ALAQVLSRNGVDLTTLPAGTASYTGSFPQPFSFTDSGLSPYTPYTYSIRATTVAGNSTSSPVSALSVTTASAPPTVAFLTTAPY ITQNSVT ( O ) DLDPCTLYSYTLTATTNDGQTFTTSAKSFTTLADKAVLSPTVTSLNYTYNAFSFAWTNSALSPCPGSGGTSGYQ LSLSVNSGAATLVNPTTTTSYSLSAGVLPSSTYAFYLRFTNTNNNVSADALLTTFTTFANTPTVTALGVTANSSNSLTEQWTGT ANGGGPLFYKVDRTSPSVLS IKNFADSLTSATDNTGLLPFTDYTYSVQARNSQTPTANLSTVATATFKTAASQATFSSSTVSST PAANS I LFSWTAATWNSLTGDYSWT ISGNPTPSPSSGVVTTNSVTVSNLRAYNQYTFTVYARSGSGNTYDSAVLTASNIQTLAA APTIVAGQFTSTGQTYNSVSLEWNGVLTLINGPTVNSASQWLVERRLNNPQGSFVSVGTFAAATLSTTDNDASLAPYTVYDYRL TVRNDQYSTTATLSSIRTAAAPPT I DPSQIEPSFSANGPTSYAVTFDLAGAYYRGTPASVSATFLLRYKTTGSGSFTSAPTFTT DTTTVTMQASQVYDLEWTITNAGPLSSTPSTGSVSVPALAPYFNAPTITTSDVTTTTADISWPAPTTKNGNDP IVYWVQYTAGQ PNPGNQTIQTSSDILQTTSTTVSGLYPGVIYTWTVSAKNANSGF SSPFSTVSS LRQDPADP IWQTAPTLSASGQTTSAFVFNWA APVYAQDDPNNLKYRVVATQITASPAFPDPTLFSKTVS INSAAVTVAAASNTGYEPYVQYNVTVHVKSSTDNFVPSNTELTVTT AGSKPSWGALTQSSLTGDRS SRQITLEWPDVPYPQDASTLTYSVSRGGTTI ISGLTTSSYVDTGLTPFTDYAYIITATNSKGST ALGTAVTFKTTEATPLFAGSETLTMTPNANGAS ITYAAPNFPYGTLSGYTVSVVDTSFTDAHVTPTQSSNSAANVFTRTITSLF PFRTYAVTI IATNGAGTS PPS DSRTGTFTTSPSAPI LDESLLDAOVT SNTS ITVSWKGPOPWERNSVLSSYRVECYMOEEDGAW TFVNAPTRTPLPADNAAYNTATQQSYQEVFSGLLPYRNYTFNVFVIGSSLTSSAIEVLARTLPGLPPTPLQATVIPISDTQARV SWPAVDDINGPIVSYVVEGQRLLPGYNETWPVPVVYAELTNGRNVTTVSGLAAESKYRFRVRTFTAAGFS LGPFGT SATTLEAA AGSQGWVAAVVLI PL I I IALVALLIVRRRQGKPMPWEPLQQRWKQRQNKDANNSMMLMGVQHQQDNMAGMPSRIVPAEELADCV AQLHANSDLGFAEEYEALNVNEEYATNAALMPANKS KNRYANI L PYDHSRVRLSVI PGVEGSDYVNANY IDGYRKS HAYIACQG PLPDTFDDFWRMVWEQRSAVIVMVTNEEEKGRVKCHRYWPDDSEVVFGDVEVRMTRQEELSEFITRVFSLKNIRTGETRTVHHL QFTGWPDHGVPHS TSS LIKFVKQAKAVQPNDAGPMVI HCSAGVGRTGTF IVTDMSLERTKVENNVDIFGCVSALRRNRMYMVQT EEQYMF IYQTLADAVANGDSEVSARDLAQHVARLAQHNKATDSSGFEEEFRLFQQPDKKKPTTDAATLSANKSKNRFATLLPCM HLNDHTRVKLNA I PGVNGSDY INASY I DGFRRKRAYIATQGPLPATVNDFWRMIWEQRVEGIVMI SREEESGRVKCHRYWPDQD VLQAGA IEVTLLSQLDRTNY I LRRFS ITNETTKETRQ I DQFQYLAWPDTS LPASTANF IDFMTQVK IWQSNANPNNPVVVHCST GAGRTGAYIVIDMAMDRLASEGVVDVFHTVKMLRSQRPNMVQALEHYEFVYSAVLEYIRSNSG 


\title{
DNA sequences catalytic domain
}

\begin{abstract}
>Spongue
AGGGATTTCCCAAGCTACGTTGAGGACATGCTTAATGATGCTGGATTTAAGTTCTCTGAGGAATACGAGAAAGT ATCAGCTGTTGGACTAGATCATTCTAAAGATGCGTCACTCCTTCCTGAGAACAGAGCTAAGAACAGATACACTA ATATATTGGCCTATGATCACTCGAGAGTCAAACTTGAGAGCATCGACGATGAACCAGGATCAGATTACATCAAC GCAAACTACATACCAGGTTACAGGATGCGTAGAGCCTATATTGCAACACAAGGACCTTTACCATCAACCTTTGA TGATTTCTGGAGGATGACATGGGAACAGAATTCGCACGTTATTGTAATGCTGACTCAGCTCGTTGAAAGAGGAA GGACGAAATGTCATCGCTATTGGCCCGGAGCACAGCCGGAAGTTTATGGAGAAATAAACGTAGACATGTTAAGC GAGACTGAGAAGAGTGACTGGATAGTGCGGAAGTTCAAGATCACAAAAGAGAAGAGAAGCCGTACAATAACACA CTACCAGTTTGTATCATGGCCTGATCATGGAGTCCCCGATGAAGCCGGACCTGCTCTGGATTTTGTACGGGAAG TACATGAAGTAGCTTCATCAGCTTTCGGACCTGTTATAGTCCACTGCAGTGCTGGTGTAGGTCGTACAGGAACA TTCATAGCACTGGGTACACTGCTCCAGCATATTAAGGACCATGACTGGGTTGACCTCTTTGGACTGGCTAGTGA AATGAGACAGCATCGTAACCACATGATCCAGACCGAGCCTCAATACGTCTTCATTCACAAAGCAATGGTTGATG CTTGCAAGAGCAGCATGA
\end{abstract}

$>$ Nematode. 2

GAAGACTTTGCCGACCACGTGAGACTAATGTCAGCGGACTCTGACTTTAGATTCTCAGAAGAATATGATATGAT GCGAAATGTTGGAGTTGGACAATCTGTAGCTGCATCGGAATTGCCAATTAACAGACCGAAAAATCGATTTACAA ACATTCCATCCTACGATCACTCGCGTGTTAAACTATCGAATCCAAATAATATCGAAGGTGGAGATTATATAAAT GCGAACTATGTACCTGGATTCTCATCACGTCGAGAGTTTATTGCTGCACAAGGTCCTCTACCAACAACACGTGA TCATTTCTGGCAGATGACGTGGGAGCAGCAGTGTCCAGCAATTATAGCACTCACGAAATGTGTTGAAAAGGGAA GAGATAAATGTCATCAATATTGGCCTGATCATGAGAATGTACCTGTTTTGTATGGAGATATTGAAGTGACAATA GTAGCAGAAAAGGAATTCGATGAATTCGTGATCCGAGATATTCGACTTGAAAAATCAGGTCCCGATGGTCGCGT AACACGATTTGTCAGGCACTGGCATTATATGGCATGGCCTGATTTTGGAGCCCCGTCTCATCCAAACGGTATCA TTCAATTCTCAAGAATGTTCCGTCACCATCTTCCACATTCACCTCATAATGCTCCAACGATAGTGCATTGCAGT GCTGGAGTTGGTCGTTCTGGAACATTTATCAGTATTGATCGTCTCCTACAATCAAGTTCATTTGGTGATCCAAT TGATGTATTTGGAACTGTTTGTGAAATGCGATACGAGAGATGTCAAATGGTACAGAATGAGCAACAATACATAT TCATTCACTATTGTATCCTTCAAGTTCTACAAGGATCA

>annelid

GTGGACTTCGTGCAGCATGTTGCAAGCATGGCAGCTGACAGTGAATTCAAATACTCAGAGGAGTATGAGGAGTT GAGCTTAGTGGGCAAAGATCAGCCTAAGGAGGCGTCCGACCTGCCCTGCAACCGTTCCAAGAACCGCTTCATCA ACATCCTACCATATGACCACAGCAGGGTGAAGTTACTCCCAATTGATGACGAAGACGGCGGAGATTATATCAAT GCTAACTGGATCCCTGGCTACAATTCGCGTCGAGAATACATCGCATCACAAGGACCTCTGCCATCCACTCGAGA TGACCACTGGCGCATGATTTGGGAGTGCAATTGTCAGTGTATTGTAATGCTCACCAAATGCGTGGAGGCAGCAA GACAACGGTGTGACCATTACTGGCCTATTGATTCTGAGGCTATCTACTACGGTGATCTTCAGGTGCAAATTCTG AATGAAACGAAATCCACAGATTGGAGCATCTCAGAATTCATCATTTCCAAAGGCGATCAATCACGACGTCTGAA GCATTTTCACTACCACTCTTGGCCCGACATGAAAGCCCCGAATAAAACGGGAAGCATCGTGCCCTTCGTGCGCC TCATTCGTGCCAAGGTTCAGTTGGATGGTGGACCGCTGCTCGTGCACTGCAGTGCTGGTGTGGGTCGCACTGGA ACCTTTATAGCTCTGGATCATTTGATGCAGCACATCGAAGAGCATGATGACATTGACATCTATGGAACCGTTTA CCAGATGCGCAAACATCGGTGTATGATGGTCCAGACTGAGTCACAATACATCTTCATACACAAGTGCTTGGAGT TCGTTCTTGAAGGGGG

$>$ FlyDPTP 4 e

AAAGACTTCTCCGAGCACTACAGGATCATGTCGGCCGATTCGGACTTTCGTTTCAGCGAGGAGTTCGAAGAGCT GAAGCATGTGGGCCGCGATCAGGCCTGCAGCTTTGCCAATCTGCCCTGCAATCGGCCCAAGAACCGATTTACCA ATATCCTGCCCTACGACCATTCCCGTTTTAAGCTCCAGCCAGTGGACGACGACGATGGTTCGGATTATATTAAT GCGAACTACATGCCGGGACACAATTCGCCGCGCGAGTTTATCGTCACCCAGGGACCGTTCCACTCGACGCGCGA GGAATTCTGGCGGATGTGCTGGGAGAGCAACTCCAGGGCGATTGTCATGCTGACGCGCTGCTTTGAGAAGGGTC GCGAGAAATGCGATCAGTACTGGCCCGTGGATCGGGTGGCCATGTTCTACGGGGACATCAAGGTGCAGTTGATT ATCGACACGCACTACCACGACTGGAGCATATCAGAGTTTATGGTCTCAAGGAACTGCGAATCGCGTATAATGCG TCACTTTCACTTCACCACATGGCCGGACTTTGGGGTTCCAGAGCCGCCGCTGTCGCTGGTTCGCTTTGTGCGCG CCTTCCGCGATGTCATCGGCACAGATATGCGTCCCATCATCGTCCATTGCAGCGCTGGAGTGGGCAGATCGGGC ACGTTTATAGCCCTGGATCGCATCCTGCAGCACATTCACAAGTCAGACTACGTGGACATTTTTGGCATCGTGTT TGCCATGCGAAAGGAGCGCGTTTTTATGGTGCAGACGGAACAGCAGTATGTGTGCATCCATCAGTGTCTGCTGG CGGTGCTGGAGGGCAAG 
$>$ Fly PTP10D

AAGAACTTTGCCGAGCACTATCGCCTAATGTCCGCCGATTCGGACTTCCGTTTCAGCGAGGAATTCGAGGAACT GAAGCACGTTGGCCGGGATCAGCCGTGCACTTTTGCCGATTTACCCTGCAATCGTCCCAAAAACAGGTTCACCA ATATACTGCCCTACGATCACTCACGTTTCAAGCTTCAGCCGGTGGACGATGATGAGGGTAGTGATTATATCAAT GCCAATTACGTGCCGGGTCACAATTCACCGCGCGAGTTCATCGTGACCCAGGGACCATTGCATTCGACACGCGA TGACTTCTGGCGAATGTGCTGGGAGAGCAACTCGCGGGCCATAGTCATGCTGACCAGGTGCTTTGAGAAGGGGC GCGAGAAGTGCGACCAGTATTGGCCAAATGATACGGTGCCCGTCTTTTACGGTGACATCAAGGTGCAGATACTC AACGACAGTCACTATGCCGACTGGGTGATGACCGAGTTCATGCTATGCAGAGGCAGCGAACAGCGCATCCTGCG ACACTTCCACTTCACCACCTGGCCGGACTTCGGTGTTCCCAATCCGCCACAGACACTGGTGCGCTTTGTGCGCG CCTTCCGCGATCGAATTGGTGCGGAACAGCGACCCATTGTGGTCCATTGTAGCGCCGGTGTGGGAAGGTCTGGC ACCTTCATCACCCTGGATCGCATCCTGCAACAGATCAACACGTCTGACTATGTGGACATATTTGGCATAGTATA TGCCATGCGCAAGGAGCGCGTTTGGATGGTGCAGACGGAGCAGCAGTATATCTGCATCCACCAGTGCCTGCTGG CGGTGCTCGAGGGGAAG

$>$ Fly $\cdot P t p 52 F$

AAGAACTTTGCCATCTTTTATACCGAAGTTGCCAAGCCGGAGAAACTGGCCAGGGAGTTCAAGGAGATCACCGT TGTGGCCTTGGAGCTCAGCTATTCCGCCTCCGAATTGGGTTGCCACAAGAATCGCTATGCGGACATATTTCCTT ATGACAAGAATCGCGTGATTCTGGACATCGATGCGGAGGGATCGGACTACATCAATGCCTCCTTCATAGATGGT CACACGCGTAAGAAGGAGTACATAGCCACCCAGGGACCAAAACCAGAGAGTGTGATGGACTTTTGGCGCATGAT CCTGCAGTACAACGTGCGGGTTATTGTTCAAGTTACCCAGTTTCGCGAGGGCAATACGATCAAGTGTCACGAGT ACTATCCGTATAATGTGCGTGGATTGACTGTGACTATCAAGTCCAAGGAGGTTCTGGAGCTGTACGATCGAACT GAGTTGACTGTTGTGCACGACAAGTACGGACTGAAGGAGAAGGTGATCCACTACTATTTCAAAAAGTGGCCCGA TCACGGCGTGCCCGAGGATCCCATGCATTTGATTATGTTTGTGAAGAAGGTGAAGGCGGAGAAGCGACCCAGTT ACTCGCCCATTGTCGTCCATTGCAGTGCGGGAGTGGGCAGGACGGGCACGTTTATTGGCCTGGATCTGATCATG CAGCGATTGAAGAGCGAGTCGAAAATCAACATTTTCGAAACGGTCAAAAAGCTGCGATTCCAGCGCATGAAAAT GGTGCAAACGCAGCAGCAGTACACATTCCTCTACGCCTGCACCTATGAGCTGGTCAAGCATAAGATA

$>$ AcornWorm

AAAGGTTTTCGTGATTATGTGAATGAATTAGACGAAGACGACAGGAAAGGATTTGAAGAGGAATATGACGATCT CCGAAAACTAGTAGTGAGACAGCCAACATCGGTGGGACAGCGAATGGAACACAGATCAAAGAACAGATACACTA ACATTGTACCCTATGATAATAACAGAGTTATATTGTCAGGCAGTGGGAATAGTTACATTAATGCCAGTTATATT TCGGGTTCCAAAGGCTCACGATCGTATATTGCAACACAAGGACCACTGGAAAGCACTTGTGGCGATTTCTGGAA AATGATTTGGCAACAACGCGTGACCACTGTCGTCATGATGACGCAATGCAACGAACTTGGAAAGAGTAAATGTC ACCACTACTGGCCACGAGATACCCAGACACAGATTTCGCACAGTGATCTCACAATAAGACTAACATCTGAAACC AGGCTTCCCGATTGGATAATACGAGACTTTTCAATAGAATCTAATGGTGAAATTCGTGCATTACGCCAGTTTCA CTTTACATCGTGGCCAATCACAGGGACGCCGTACGATGCTGACCCATTGATCCGATTTATTGAAGCTATTCGAA TTCAAGTCTTGCCAAATAGCGGACCAATACTAGTACACTGCAGTGCTGGTGTTGGTCGAACAGGTGTCTTTATT GCATTGTATCACCTTCTAGAATATTTCTACACTATGATCCAGGTTGATATATTTGGTAAAGTCATAAAAATGAG GAAACAAAGACCATTTATGGTTCAGACACAGGGTCAGTATGAATTCCTCTACTATGCAATTCAACAACATATAA AAGAGAAG

>SeaUrchin.1

AATAAGTTTGAGGGGCATTACGGCCGGATGAAGGCTGATTCAGATTTCAGATTCACAGAGGAATATGATGAAAT CCGTCTAGTGGGTAAGGACCAAGCCATTGTATCAGCTTTAGAGATGGTGAACAGAGCCAAAAACCGATTCACTA ATATCCTACCATATGAGCATTCCCGCGTTAAGCTTGCGGCTCTCGCCGACGATTCAGACACGGACTACATCAAT GCAAATTACATCCCCGGTTACAACTCGCCACGCGAATTCATGGCTTGCCAGGGCCCGTTGCCGGGGACTGTGGA TGATATGTGGAGGATGATCTGGGAGAAGAAGACCTCCATCATCGTCATGCTGACGCAGCTCGTTGAAAAGGGCA AGATAAAATGTCATGAATATTGGCCTGCCGATTACAACCCCGTCACATATGGATCGATACAGGTCTCCGTACAA GCACTTCAGCAGTATGACCACTGGGTCATTAGGGAGTTCAGTATATCACAGGGAGATGAAATTCGAAAGCTTAC GCAGTATCACTTTATGTCATGGCCTGACCACGGGGTCCCGGAGAAGACCTGGACCATGCTGGACTTTGTCCGAA CGGTCCGAGAAGCAATTCAGAAAACAGCCAGCGATCGGCCTATTGTAGCGCACTGCAGCGCCGGTGTTGGTCGG ACAGGGACGTACATCGCTCTCGATCGGTTGATGCAGGCCATGCAGGAGAACGACTACATCGATATCTTTGGGAT TATATGTGAGATGAGGATGCAGAGAAATCACATGGTCCAGACGGAAAAACAGTACATCTTCATTCACGAATGTG TGATGGATCTCTTGCGGAGAGG

>SeaUrchin.3

AGGAAGTTCCCTGACCATGTCAATCGCATGAGTGCTGGCAACAATACTGGATTTGTGGAAGAGTACAATTCTCT ATCAAATGTGGGCAGAGACAAGAGCACCGATGCTTCAAGGCTTGCTGTCAATGCAACCAAGAACAGATACAGGA ACATCCTACCATTTGATGAGACTGCCGTTCATCTACAAGATTTGGAGGAAGGACAGACCAGTGACTACATCAAT GCAAACTACATCCAGGGCTTGCACTCTATGAAGGAATACATTGCTTCCCAGGGCCCCGTACCAGACTCTGTCAA CGATTTCTGGGAGATGGTGTTTGAGAATAAGAGCACAACCATCGCAATGATCACTGGGCTTGTTGAAGGAGGAA AGACCAAGTGTGAGCACTACTGGCCAGATGACGATACCCCAGTGAACTACGGCAGTGTGACGGTCACCATGACC 
CATACACAGGAGATGGAACAATGGACCGTCAGGTCATTCCTGCTAGAGAAGGGCATGCAACAGTTTGAGACGCA TCAGTACGCCTTCAAGGGCTGGGTCGATCATGATGTACCCAACAATGCTCGTCCTATGATTGAATTCATCCGGA CCATAGACGTCGCCCATGATGCTAATCTAGGACCCATCACCGTGCACTGCAGTGCTGGTATTGGTCGAACCGGT GTGTTCATTGCCCTTCACAAGCTGATCAAGCAGGTTGAGACTTCCAAGCCCAATGACTACATCGATGTGTGCGG CACTGTGGCACGCATGAGGGAGCAACGTTTCAACATGGTCCAGACAGTCAAACAATACATGTTCATCCACCAGT GTCTGCTACTTCTTATGAGA

>SeaUrchin. 4

AATATGTTTGCCCAACACGTTGCCAAGATGTCTGCTCGCAACAATGCTGGATTCAGTGATGAATATAATAATTT GCCAGCGATGGAAAAGACTCGAGCGGTGACTGCGGCTCGACAGGCCAGAAATGATGATAAGAACAGATACAGGA ACATCTTGCCTTATGACTACACCAGAGTCCAACTGAAAGGAGTAGCCGGTACCGATTACATCAATGCAAGTTAC ATTAAGGATGATCATGGACAGAAGAAGTACATTGCGACCCAAGGACCCTTGCCAAACACCATTGAACACTTCTG GGAGATGGTTTGGGAAAACCAGACCAGTACCATTGTGATGATGACTGCATTGGTGGAAGGAGGAAAGACCAAAT GCGAGCACTACTGGCCAGCTGGAGAAGAGCCTCAACTGCATGGCAACGTGACTGTCACCCTTGTTGGCAGTAAC CAGACCGATAACTTCATCGAACGCACACTTCTCCTAGAAAAGGAGGGTACAGAACGCACCGTGACCCACTACCA GTACCTCGCCTGGACCGACCATGGCGTGCCCGAGTCCACTGCCCCTCTAGTGGGGCTACTCCGTCAGGTCAAAG TGACTAACCAGGAAGATGGGGCAGCAACCGGTCCGATCGTCGTCCACTGCAGTGCTGGTGTTGGCAGGACAGGA ACGTTCATCGCTGCTGATATGTTAATGGATGCCATACAACGATCCACTGCTACCGACTACATTGACGTTGCTGG TACTATAGCGAAGATCAGAGAGCAAAGAGCCCTCTTGGTTCAAACACTGTACCAATACATCTATCTTCATCGTA CCGTGCTTTCCTTGATCGAGGAGCAA

$>$ Ciona

GACGAATTTCCAAATCACGTTTCATCCTTAAGTCAAAATAAAAATAAAGGATTCAGTCTTGAATTTGATGATAT TAGGGGAATTCCTTATGCTGGTACTACGGCTATTGCCGAAAACTCTTGTAATAAAACAAAGAACAGAACAACGA AATTAGTCCCATTTGACCACTGTCGGGTTAAAATTGAAGGAATCCCAGCGATACAAGGTTCAAATTATATCAAC GCATCGTACATACCAGGTCTAGACA GTCCGGAGCAATACATAGCAACACAGACTCCGTTAGACCACACAAAGAA AGATTTCTGGCGAATGCTTTGGGAGACGGGCTCAACTAACATTGTTATGTTGTGCGGTTCTGTGAATGCAGGCA AAAAACGCTGCGACGAGTTCTGGCCCAAGAAACAAACTGAATATTTTGGAAACCTCGCTGTTCAGATGAAGGAA GAGATACGACACGATGAATGGATTATTCGGCAATTCATCGTCACAATGCGAGACAAAGTTCGACACGTGACACA ACATCACTTTATAAAATGGCCGCAACTGGAACAAGCTGAAAACTCTTTGCCACTGGTTCGATTCATAAAGAACT ACCGATTGTTACGGGACAGAAGCATCAATCCAACTATTGTCATGTGCAGCAACGGGAGTGGTCGATGCGGAGTC TTCATCGGATTACTTCGAATATTAGACAACAACGGACGTGACGTCGATGTGTTCGGAACGGTTGCTGCATTGAG GAAATACAGACCATATATGGTGCAGACACTGTCCGAGTACATATACCTGCACCAATGCGTGCTCAAGTTTATCG ATGACGTCAC

>PTPRQhuman

AAATCCTTCCTGCAACATGTTGAAGAGCTTTGCACAAACAACAACCTAAAGTTTCAAGAAGAATTTTCGGAATT ACCAAAATTTCTTCAGGATCTTTCTTCAACTGATGCTGATCTGCCTTGGAATAGAGCAAAAAACCGCTTCCCAA ACATAAAACCATATAATAATAACAGAGTAAAGCTGATAGCTGACGCTAGTGTTCCAGGTTCGGATTATATTAAT GCCAGCTATATTTCTGGTTATTTATGTCCAAATGAATTTATTGCTACTCAAGGTCCACTACCAGGAACAGTTGG AGATTTTTGGAGAATGGTGTGGGAAACCAGAGCAAAAACATTAGTAATGCTAACACAGTGTTTTGAAAAAGGAC GGATCAGATGCCATCAGTATTGGCCAGAGGACAACAAGCCAGTTACTGTCTTTGGAGATATAGTGATTACAAAG CTAATGGAGGATGTTCAAATAGATTGGACTATCAGGGATCTGAAAATTGAAAGGCATGGGGATTGCATGACTGT TCGACAGTGTAACTTTACTGCCTGGCCAGAGCATGGGGTTCCTGAGAACAGCGCCCCTCTAATTCACTTTGTGA AGTTGGTTCGAGCAAGCAGGGCACATGACACCACACCTATGATTGTTCACTGCAGTGCTGGAGTTGGAAGAACT GGAGTTTTTATTGCTCTGGACCATTTAACACAACATATAAATGACCATGATTTTGTGGATATATATGGACTAGT AGCTGAACTGAGAAGTGAAAGAATGTGCATGGTGCAGAATCTGGCACAGTATATCTTTTTACACCAGTGCATTC TGGATCTCTTATCAAATAAG

$>$ PTPRQ.mouse

AAATCCTTCCTGCAACATGTTGAAGAGCTTTGCACAAACAACAACCTAAAGTTTCAAGAGGAATTTTCGGAATT ACCAAAATTTCTTCAAGATCTCTCCTCAACTGATGCTGATCTCCCTTGGAACAGAGCAAAAAATCGCTTTCCCA ACATAAAACCATACAATAATAACAGAGTGAAGCTGATAGCTGATGTAAGCATCCCGGGATCAGATTACATTAAT GCCAGCTATGTTTCTGGATACTTATGTCCCAATGAGTTTATTGCCACCCAAGGCCCATTACCGGGAACAGTTGG AGACTTTTGGAGAATGGTGTGGGAAACCAGAGCGAAAACATTGGTAATGCTCACACAGTGTTTTGAGAAAGGAC GGATCAGATGCCATCAGTATTGGCCCGAGGACAACAAGCCAGTGACAGTCTTTGGAGACATACTGATTACAAAG CTTATGGAGGACATTCAGATCGACTGGACCATCAGGGATCTGAAAATTGAAAGGCATGGAGACTGCATGACTGT CAGGCAGTGTAACTTCACGGGTTGGCCTGAGCATGGGGTTCCTGAAAACACCACCCCTCTAATTCACTTTGTGA AGTTGGTACGAACAAGCAGAGCACACGACGCTACCCCTATGGTTGTGCATTGCAGCGCTGGGGTTGGAAGGACG GGAGTTTTCATTGCTCTGGACCACTTAACACAGCACATACATGACCATGACTTTGTGGACATATATGGACTAGT AGCTGAACTGAGGAGTGAGAGAATGTGCATGGTGCAGAACCTGGCACAGTATATCTTCTTACACCAGTGCATTC TGGATCTTTTATCAAATAAA 
$>$ PTPRQ. chiken

AAATCCTTCCTACAACACGTTGAAGAGCTTTGTACAAACAACAACCTAAAGTTTCAGGAAGAATTTTCGGAACT TCCTAAGTTTCTTGAAGACCTTGCTTCAACTGATGCTGATCTGCCTTGGAACAGGTCTAAGAACCGTTTCCCAA ACATTAAGCCATATAATAACAACAGAGTAAAGCTGATGCCTGATGCTGGTATTCCTGGATCTGACTACATTAAT GCCAGCTATGTATCTGGCTACTTATGTCCAAATGAATTATTGCAACTCAGGGACCGTTACCAGGAACAGTGGG TGATTTCTGAGAATGGTGTGGGAGACTAGAGCAAAAACTCTAGTGATGCTTACACAGTGTTTTGAAAAAGGAC GGATAAGATGCCATCAGTACTGGCCAGAAGATAATAAACCAGTGACTGTGTTTGGGGATATAGTAATTACTAAA TTGGTGGAAGATATTCAAATAGACTGGACCATCAGAGATCTAAAAATTGAAAGGCATGGAGACTGCATGATGGT CAGGCAGTGTAACTTCACTTCATGGCCAGAACATGGAGTCCCTGAAACTACTGCACCAATTATCCACTTTGTGA AACTTATCCGTGCAAGTCGAGCACACGATAATACGCCGATGGTGGTTCACTGCAGTGCTGGTGTTGGAAGAACA GGTGTTTATATTGCTCTTGACCATTTAACGCAACATATAAATGACCATGATTTTGTGGATATCTATGGCCTTGT AGCTGAGCTAAGAAGTGAAAGAATGTGCATGGTGCAGAATCTGGCACAGTACATCTTTTTGCATCAGTGTGTCC TGGATCTGCTGACAAGCAGG

$>$ PTPRQ.fish

AAATCGTTTCTTCAGCATGTGGAAGATCTTTGTGCCAACGACAACGCCAAGTTTCAGGAGGAGTTTGCTGAGTT GCCGAAGCTGCTGCAGGATCTGGCCACATCAGACGCTGACCTGCCTTGGAATCGCTCCAAAAATCGCTTCACAA ACATCAAGCCCTACAACAATAGCCGAGTGAAGTTGCTGTCTGAGCCAGGAATGCCGGGTTCTGACTACATCAAT GCCAGCTTCGTCTCTGGCTATCTGTGTCCTAATGAGTTTATAGCCACTCAGGGCCCTCTGCCGAGCACCGTCGC A.ATTTCTGGAGGATGATTTGGGAAACTGGGACTAAAACCATCGTCATGCTTACTCAATGCTTTGAGAAGGGCC GGATCCGCTGTCATCAGTACTGGCCGGAGGACAATAAACCAGTCACTGTTTTTGCTGACATCATAATCACTAAA CTCACAGAGGACGTGCGTCCTGATTGGACGGTGCGGGCACTCAAAGTGGAGCGGCATGGCAGCTACATGATTGT CCATCACTTTAACTACACCTCATGGCCAGAACACGGCGTTCCCGAATCCAGCTCCACACTTGTCCAGTTTGTCA AAGCCGTTCGCTCCAACAGAGGCCATGAAAACACTACCATCGTGGTCCACTGCAGTGCTGGTGTGGGCAGGACT GGAGTGTTTATAGCACTGGATCATCTCATCCAGCATTTGCGAGATCATGAATTTGTTGACATATACGGCCTGGT CGCAGAGCTGCGCAGTGAGAGGATGTGTATGGTGCAAAACCTGGCTCAATACATGTTTCTCCATCAGAGCACTC TGGATCTGCTGTCGGCCAAA

$>$ PTPRB. human

AATCAGTTTGAAGGGCATTTCATGAAGCTACAGGCTGACTCCAACTACCTTCTATCCAAGGAATACGAGGAGTT AAAAGACGTGGGCCGAAACCAGTCATGTGACATTGCACTCTTGCCGGAGAATAGAGGGAAAAATCGATACAACA ATATATTGCCCTATGATGCCACGCGAGTGAAGCTCTCCAATGTAGATGATGATCCTTGCTCTGACTACATCAAT GCCAGCTACATCCCTGGCAACAACTTCAGAAGAGAATACATTGTCACTCAGGGACCGCTTCCTGGCACCAAGGA TGACTTCTGGAAAATGGTGTGGGAACAAAACGTTCACAACATCGTCATGGTGACCCAGTGTGTTGAGAAGGGCC GAGTAAAGTGTGATCATTACTGGCCAGCGGACCAGGATTCCCTCTACTATGGGGACCTCATCCTGCAGATGCTC TCAGAGTCCGTCCTGCCTGAGTGGACCATCCGGGAGTTTAAGATATGCGGTGAGGAACAGCTTGATGCACACAG ACTCATCCGCCACTTTCACTATACGGTGTGGCCAGACCATGGAGTCCCAGAAACCACCCAGTCTCTGATCCAGT TTGTGAGAACTGTCAGGGACTACATCAACAGAAGCCCGGGTGCTGGGCCCACTGTGGTGCACTGCAGTGCTGGT GTGGGTAGGACTGGAACCTTTATTGCATTGGACCGAATCCTCCAGCAGTTAGACTCCAAAGACTCTGTGGACAT TTATGGAGCAGTGCACGACCTAAGACTTCACAGGGTTCACATGGTCCAGACTGAGTGTCAGTATGTCTACCTAC ATCAGTGTGTAAGAGATGTCCTCAGAGCAAGA

$>$ PTPRB.mouse

AATCAGTTTGAAGGGCATTTCATGAAGCTGCAGGCAGACTCCAACTACCTTCTATCCAAGGAATATGAGGACTT AAAAGACGTGGGTAGAAGCCAGTCATGTGACATTGCCCTCTTGCCTGAGAATCGAGGGAAAAATCGATACAACA ACATATTGCCTTATGATGCCTCAAGAGTGAAGCTCTGCAATGTCGATGACGACCCTTGCTCTGACTACATCAAC GCCAGCTACATCCCCGGTAACAACTTCAGACGAGAATACATCGCCACTCAGGGACCGCTTCCAGGCACCAAGGA TGACTTCTGGAAGATGGCGTGGGAGCAGAACGTTCACAACATCGTCATGGTGACCCAGTGTGTTGAAAAGGGCC GAGTGAAGTGTGACCATTACTGGCCAGCAGACCAGGACCCCCTCTACTACGGTGATCTCATCCTACAGATGGTC TCGGAGTCCGTGCTCCCCGAGTGGACCATCAGGGAGTTTAAGATATGCAGTGAAGAACAGTTGGATGCACACAG ACTCATCCGTCACTTTCACTACACGGTGTGGCCAGACCATGGGGTCCCAGAGACCACCCAGTCCCTGATCCAAT TTGTGAGGACAGTCAGGGACTACATCAACAGAAGCCCCGGGGCTGGGCCCACCGTAGTGCACTGCAGCGCTGGT GTGGGCAGAACAGGGACGTTCGTTGCCCTGGACCGGATCCTCCAGCAGTTGGACTCTAAGGACTCCGTGGACAT TTATGGGGCAGTGCATGACCTAAGACTCCACAGGGTTCACATGGTCCAGACCGAGTGTCAATATGTGTATCTGC ATCAGTGTGTAAGAGACGTCCTCAGAGCAAAG 
$>$ PTPRBgallus

AGTCATTTTGAAGCACACTTCACCAAACTCCAAGCAGACTCCAACTACCTCCTGTCCAAGGAATATGAGGACTT AAAAGATGTGGGTCGAAACCAGACATGTGACATAGCACTTCTGCCAGAGAACAGAGGGAAAAATCGATACAATA ATATATTGCCTTATGATACATCAAGAGTGAAGCTTTCCAATGTGGATGATGACCCTTGCTCAGACTACATTAAT GCAAGCTATATACCAGGCAATAATTTCCGCAGGGAATACATAGCAACTCAGGGCCCTTTGCCTGGCACTAAAGA TGAGTTCTGGAAGATGGCATGGGAACAAAATGTGCACAATATTGTCATGGTAACCCAGTGTGTTGAGAAGGGCC GGGTAAAGTGTGATCACTACTGGCCCCTTGACCAGGATTCCTTGTACTATGGAGACCTGATAGTTGAGATGCTG TCTGAATCAGTGCTCCCAGAATGGACAATACGAGAGTTTAAAATCTGCAGTGAGGAGCAGCTTGACTCAACGAG GCTCATTCGTCACTTCCACTACACTGTGTGGCCAGATCACGGAGTGCCAGAGACCACCCAGTCCCTGATCCAAT TTGTCAGAACTGTAAGGGATTATATCAACAGGACCCCAGACACGGGACCAACTGTTGTGCACTGCAGTGCTGGT GTTGGCAGAACTGGCACATTCATTGCACTGGACCGAATTCTGCAGCAACTGGATTCAAAGGACACTGTTGACAT TTATGCAGCAGTGCACGATCTAAGGCTTCACCGCGTTCACATGGTTCAAACAGAGTGTCAATACGTGTATCTAC ATCAGTGTGTGAGAGATGTGTTAAGAGCCAGA

$>$ PTPRB.frog

GCCCAGTTTGAAGAACATTTCAGCAAGCTTCAGACCGATTCCAATTACTTGCTTTCTCGGGAGTATGAGAACCT TAAAGATTTTGGTCGAGACCAGTCTTCTGATACTGCCCTTCTCCCAGAAAACAGAGGAAAGAATAGATATAGCA ACATATTGCCCTATGATTCCACAAGAGTGAAACTTGCCAACGTTGACGATGACCCCTGTTCAGACTATATTAAC GCCAGCTATATGCCAGGCATCAACTTTCGGAGAGAATATATTGCTACACAAGGGCCTTTACCAGCAACCAAAGA TGATTTCTGGAAAATGGTGTGGGAACAAAATGTTCACATCATTGTGATGGTGACACAGTGTACAGAGAGAGGAC GGGCAAAATGCGATCATTATTGGCCCATGGACCAAGACTCATACTATTACGGAGACCTGATAGTACAAATGCTG TCAGAATCTGTGCTTCCTGAATGGACAATAAGGGAATTTAAAATCTGCAGTGAAGATCAAATTGATGCACCACG ACTAGTCCGTCATTTCCACTATACAGTGTGGCCAGATCATGGGGTGCCAGAAACCACCCAATCACTGATCCAGT TTGTAAGAACAGTCAGAGACTATATTAACAGAACTCCTGGAAGTGGCCCTACTGTGGTGCACTGCAGTGCTGGT GTTGGCAGAACTGGCACATTCATTGTACTGGACCGAATGCTTCAACAAGTTGATACAGTGGATTCAGTGGATAT ATTTGGAGCTGTTAGGGACCTCCGAATTCACCGGATGTACATGGTGCAGACCGAGTGTCAGTACGTCTATTTGT ATCAATGTGTAAGAGACGTCTTAAGAGCCAGG

$>$ PTPRB.fish

GCCAATTTCGAGTCCCACCTTGCTAAACTTCAATCCGATTCCAGTTTCCTTCTGTCAGAAGAGTTTGAGGGCCT AAAGGACGTGGGACGGATCCAAACTCAAAATGCTGCACGTCTGCTTGGGAACCGCAACAAAAACCGATACAACA ATATACTTCCCTACGACTCCACGCGGGTGAGACTGTCCTGTCTGGAGGATGACCCCTGCTCAGACTACATCAAC GCTAACTATATACCTGGAAACAATTTCAGATGGGAGTATATCGCCACACAAGGGCCTTTGCCAGGTACCAAGGA CGATTTTTGGCGAATGGTGTGGGAACAAAATGTGCACAGCCTAGTAATGGTGACGCAGTGTGTGGAAAGAGGCA TGGTAAAGTGTGACCACTACTGGCCGACGAACAGCGAGCCTTTGTGCTATGGAGACATTGTGGTGCAGCTGCTT TCTGAGAAGGTCTTTCCAGAGTGGACCATTCGGGACTTCAAAATATCATGCGAGAGTCAGCTGAGATACCCCAG GATGGTGCGTCAGTTCCATTACACCATCTGGCCAGATCACGGCGTCCCGGACACCACTCAGTCTCTTATCCAGT TTGTGCGAACAGTCCGAGATTTCATCAACAGGACAAACAGTCCTGGAATCTCCGTAGTACACTGCAGTGCTGGT GTGGGTCGAACTGGGACTTTCATAGTGCTGGATCGTGCTTTACAGCAACTAGACAGGAACTGCACAGTGGACAT TTACGGCTGTGTGTTTGACCTGCGCCTGCATCGCTCATACATGGTACAAACCGAGTGTCAGTATGCTTATATCC ATCAGTGTGTGCGGGATGTTTTGAGGGCTCGC

>PTPRJ.human

GAGAATTTTGAGGCCTACTTCAAGAAGCAGCAAGCTGACTCCAACTGTGGGTTCGCAGAGGAATACGAAGATCT GAAGCTTGTTGGAATTAGTCAACCTAAATATGCAGCAGAACTGGCTGAGAATAGAGGAAAGAATCGCTATAATA ATGTTCTGCCCTATGATATTTCCCGTGTCAAACTTTCGGTCCAGACCCATTCAACGGATGACTACATCAATGCC AACTACATGCCTGGCTACCACTCCAAGAAAGATTTTATTGCCACACAAGGACCTTTACCGAACACTTTGAAAGA TTTTTGGCGTATGGTTTGGGAGAAAAATGTATATGCCATCATTATGTTGACTAAATGTGTTGAACAGGGAAGAA CCAAATGTGAGGAGTATTGGCCCTCCAAGCAGGCTCAGGACTATGGAGACATAACTGTGGCAATGACATCAGAA ATTGTTCTTCCGGAATGGACCATCAGAGATTTCACAGTGAAAAATATCCAGACAAGTGAGAGTCACCCTCTGAG ACAGTTCCATTTCACCTCCTGGCCAGACCACGGTGTTCCCGACACCACTGACCTGCTCATCAACTTCCGGTACC TCGTTCGTGACTACATGAAGCAGAGTCCTCCCGAATCGCCGATTCTGGTGCATTGCAGTGCTGGGGTCGGAAGG ACGGGCACTTTCATTGCCATTGATCGTCTCATCTACCAGATAGAGAATGAGAACACCGTGGATGTGTATGGGAT TGTGTATGACCTTCGAATGCATAGGCCTTTAATGGTGCAGACAGAGGACCAGTATGTTTTCCTCAATCAGTGTG TTTTGGATATTGTCAGATCCCAG

>PTPRJmouse

GAGAATTTTGAGGCCTACTTTAAGAAGCAGCAAGCTGACTCTAACTGTGGGTTTGCAGAGGAATATGAGGACCT GAAGCTGATTGGGATAAGTTTACCTAAATACACAGCTGAGATAGCCGAGAACAGAGGGAAGAACCGCTACAACA ATGTTCTGCCCTATGATATTTCTCGAGTCAAACTTTCAGTCCAGACCCATTCGACAGATGACTACATCAATGCC AACTATATGCCTGGCTACCATTCCAAGAAAGATTTCATTGCCACACAAGGACCTTTACCCAACACTTTGAAAGA TTTCTGGCGTATGGTTTGGGAGAAAAACGTATATGCCATTGTTATGTTGACCAAATGCGTGGAGCAGGGAAGGA 
CCAAATGTGAGGAGTACTGGCCTTCCAAGCAGGCTCAGGACTACGGGGACATAACTGTGGCGATGACATCAGAA GTCGTTCTTCCAGAATGGACCATCAGAGATTTTGTGGTGAAAAATATGCAGAATAGCGAGAGCCATCCTCTGCG GCAGTTCCATTTCACCTCCTGGCCTGACCACGGTGTTCCTGACACCACTGACCTGCTCATCAACTTTCGGTACC TGGTCCGGGATTACATGAAGCAGATACCCCCCGAGTCACCAATTCTGGTGCATTGCAGTGCTGGGGTTGGAAGG ACGGGCACTTTCATCGCCATCGATCGCCTGATCTATCAGATAGAGAATGAGAACACCGTGGACGTGTATGGGAT TGTCTATGACCTTCGGATGCACAGGCCTCTGATGGTGCAGACAGAGGACCAGTATGTTTTCCTCAATCAGTGTG TTTTGGATATTATCAGAGCCCAG

>PTPRJ.chiken

GAGAACTTTGAGTCCTACTTTAAGAAGCAGCAAGCAGACTCCAACTGTGGTTTTGCTGAAGAGTATGAGGAACT CAAGTCTGCTGGTGTTCATCAGCCCAAATTTGCTGCTGAAATCGCTGAGAACAGGGGGAAAAATAGATACAACA ATGTCTTACCATATGATATTTCTCGTGTTAAACTTTCAAATCCAAGCTGTACAACTGATGATTATATTAATGCA AACTATATGCCTGGATACAGCTCAAAGAAGGCATTTATTGCTGCACAAGGCCCTTTACCCAATACTATAGAAGA CTTCTGGCGCATGATTTGGGAAAAGAATATCTACTCTATTGTCATGTTGACAAAATGTGTTGAACAGGCCCGGA CGAAATGTGAGCAATATTGGCCAGACAAACAATCCAAGAGTTATGGTGACATTATTGTGACAATGGTCTCAGAA GTTGTTCTTCCAGAATGGACGATAAGGGACTTCAATGTAGAGAACGCTGACACAATGGAGAGCCACACAGTGCG TCAGTTCCATTTCACATCCTGGCCAGATCATGGAGTGCCAGAGACAACAGACTTGCTAATCAACTTCAGACACC TTGTTCATGAATACAGCAGTCAGAATCCTATTGACTCTCCTATTCTAGTGCACTGCAGTGCTGGTGTCGGGAGG ACAGGTACGTTTATTGCCATTGACCGCCTGATTCAGCAGATTGAAATGGAGAATACTGTGGATGTGTATGGAGT GGTGTACGATCTTCGAATGCATCGACCTTTAATGGTGCAAACTGAGGACCAGTATGTCTTCCTAAACCAGTGTG TCATGGATATTATTAGATCACAG

PPTPRJ.fish

GAAGATTATGAGGCTTACTATAAGAGACAAAGAGCTGATTCTTTCTGTGGATTTGCTGAAGAATTTGAGGATCT AAGGCCGGTTGGGATAAATCAATCAAAGACTGTTGCTGTGTTTCCTGAAAACAAAGCCAAGAATCGATATAATA ATGTGCTGCCATACGATTCTTCTCGTGTCAAGCTCTCTGTTCTCAGCAGCCCATTTGATGACTACATCAATGCC AACTACATGCCGGGTTACATTACAAAGAAGGAGTTTATCGCAGCACAAGGTCCATTGCCCGGCACTGTTAATGA CTTCTGGAGGCTGATCTGGGAGAAGAATGTGCACACTATTGTCATGCTCACCAAATGCAATGAACAAGGCCGGG TAAAATGTGAGGAATACTGGCCAGCAGAGATGAAGACTTTTAGCAATCTGACAGTGACAACAATTTCTGATATC CCATTGGAGGATTGGACTTTACGTGACTTTGAAGTGAAAAATATGAAAACAGCAGAAATTCGATCGGTTCGTCA СTTCCATTTCACTGCATGGCCAGACCATGGAGTTCCTGAGACCACTGAGCTTCTCATCAACTTCAGACATCTGG TCCGAGAGCACATGGATGAGTACTCAAGACATTCCCCCACACTTGTGCACTGCAGTGCGGGTGTGGGCCGGACA GGTACCTTCATTGCCATTGATAGGTTAATCTTCCAGATCGAGCGGGATGGTGTAGTGGATGTATATGGGATCAT CCATGACTTACGCATGCACAGACCCCTCATGGTGCAAACAGAGGATCAATATGTCTTTCTTAACCAGTGTGCCA TGGACATCATCAAGTCTCGA

$>$ PTPRO.human

GATGACTTTGATGCCTATATTAAGGATATGGCCAAAGACTCTGACTATAAATTTTCTCTTCAGTTTGAGGAGTT GAAATTGATTGGACTGGATATCCCACACTTTGCTGCAGATCTTCCACTGAATCGATGTAAAAACCGTTACACAA ACATCCTACCATATGACTTCAGCCGTGTGAGATTAGTCTCCATGAATGAAGAGGAAGGTGCAGACTACATCAAT GCCAACTATATTCCTGGATACAACTCACCCCAGGAGTATATTGCCACCCAGGGGCCACTGCCTGAAACCAGAAA TGACTTCTGGAAGATGGTCCTGCAACAAAAGTCTCAGATTATTGTCATGCTCACTCAGTGTAATGAGAAAAGGA GGGTGAAATGTGACCATTACTGGCCATTCACGGAAGAACCTATAGCCTATGGAGACATCACTGTGGAGATGATT TCAGAGGAAGAGCAGGACGACTGGGCCTGTAGACACTTCCGGATCAACTATGCTGACGAGATGCAGGATGTGAT GCATTTTAACTACACTGCATGGCCTGATCATGGTGTGCCCACAGCAAATGCTGCAGAAAGTATCCTGCAGTTTG TACACATGGTCCGACAGCAAGCTACCAAGAGCAAAGGTCCCATGATCATTCACTGCAGTGCTGGCGTGGGACGG ACAGGAACATTCATTGCCCTGGACAGGCTCTTGCAGCACATTCGGGATCATGAGTTTGTTGACATCTTAGGGCT GGTGTCAGAAATGAGGTCATACCGGATGTCTATGGTACAGACAGAGGAGCAGTACATTTTTATCCATCAGTGTG TGCAACTGATGTGGATGAAGAAG

>PTPRO.mouse

GATGACTTCGATTCTTACATCAAGGATATGGCCAAGGACTCGGACTATAAATTCTCTCTTCAGTTTGAGGAGTT GAAGTTGATTGGACTGGATATTCCGCACTTTGCTGCAGATCTACCGCTGAACCGATGTAAAAACCGCTACACAA ACATCCTGCCGTATGACTTTAGCCGGGTGAGGCTAGTCTCCATGAACGAAGAGGAAGGAGCAGACTACATTAAT GCCAACTATATTCCTGGATACAACTCACCCCAGGAGTACATTGCCACCCAGGGTCCCCTGCCAGAAACCAGAAA TGACTTCTGGAAGATGGTCCTACAACAGAAGTCCCACATCATCGTCATGCTCACTCAATGCAATGAGAAAAGGA GGGTAAAATGTGACCACTACTGGCCATTCACAGAAGAACCCATTGCTTATGGGGACATCACCGTGGAGATGGTC TCTGAGGAAGAGGAGGAGGACTGGGCCAGTAGACACTTCCGGATCAACTATGCGGACGAAGCGCAGGACGTGAT GCATTTTAACTACACAGCCTGGCCCGATCACGGCGTGCCTCCAGCAAACGCCGCCGAGAGCATCCTGCAGTTTG TGTTCACAGTGCGACAGCAAGCCGCCAAGAGCAAAGGGCCCATGATCATCCACTGCAGTGCGGGTGTGGGACGG ACAGGAACCTTCATTGCCCTGGACAGGCTCCTGCAACACATTCGAGATCATGAATTTGTGGACATCTTAGGGCT GGTATCAGAGATGCGCTCATACCGAATGTCAATGGTACAGACAGAGGAGCAGTACATTTTTATCCATCAGTGTG TGCAGCTGATGTGGCTGAGGAAG 
$>$ PTPRO. chiken

GATGATTTTGATGGCTACATCAAGGATATGGCCAAAGATTCTGATTATAAATTTTCTCTGCAATTTGAGGAGCT AAAACTGATTGGGCTTGATATACCTCACTTTGCCGCTGATCTTCCCATGAATCGCTGCAAAAATCGCTACACAA ATATCCTGCCATATGACTTTAGTCGCGTCCGGTTAGTCTCAATGAATGAAGAAGAGGGATCTGACTACATTAAT GCCAACTATATTCCTGGTTATAATTCACCCCAGGAATACATAGCAACCCAAGGACCACTGCCAGAAACTAGGAA TGATTTTTGGAAGATGGTTCTTCAGCAAAAATCTCAAATTATTGTTATGCTCACTCAGTGCAATGAGAAAAGGC GGGTGAAATGTGATCATTACTGGCCTTTTACAGAAGATCCTATTGCATACGGGGACATTACTGTCGAGATGCTG TCTGAGGAAGAACACACAGATTGGGTTTATAGGAATTTCCGAATTAGCTATGCTGATGAGGTGCAAGACGTGAT GCATTTTAACTACACTGCCTGGCCAGACCATGGTGTCCCCACAGCCAATGCCGCCGAAAGCATCCTGCAGTTCG TGCAGATGGTCAGGCAGAAGTCAGTAAAGAGTAAAGGCCCCATGATCATACACTGCAGTGCTGGAGTGGGACGA ACAGGAACATTCATAGCCCTGGACTGGCTCTTACAGCACATCCGTGACCATGAGTTTGTAGATATATTGGGCCT GGTGTCTGACATGAGGTCCTACAGAATGTCCATGGTGCAGACAGAGGAACAATACATTTTTATTCACCAGTGTG TGCAATTGATGTGGCAAAAGAAG

$>$ PTPRO.frog

GATGACTTTGATTTATACATAAAGGAAATGTCCAAAGACTCTGATTACAAATTCTCCCTCCAGTTTGAAGAACT AAAGATGGTCGGACTGGATATTCCGCACTTTGCCGCCGACCTTCCATCAAACAGAGGCAAAAACCGCTACACGA ATATCCTGCCATATGATTGCAGCCGCGTGAAACTGATTTCTCTAGAAGCCGATGAAGGAGCCGATTATATCAAC GCCAATTACATTCCGGGCTACAGTGCCGCCCAGGAATATATCGCCACTCAGGGGCCCCTGCCGGAGACCAGGAA CGACTTCTGGAAAATGATCCTTCAGCAGAAGTGCCAAGTTATTGTCATGCTGACCCAGTGCAACGAGAAGCGGC GGATTAAATGTGACCACTACTGGCCATTCACTCCGGAACCGGTAAACTATGGCGATATCACCGTGGAGATGGCG TCAGAGGAGGAGCAATCGGACTGGGCGCAGCGAGTATTTAGGGTCTCGTATGCGGATGAGACCCAGTGCGTCAC ACACTTTAACTTCACGGCGTGGCCTGACCATGGGGTTCCGACAGTCAATGCGGCCGAGAGTGTCCTGCAGTTTG TCCAGGTCGTCAGGCAGAAGGCGCTGAAGTCTAAGGGACCCGTAACGGTGCATTGCAGCGCCGGCGTGGGTCGG ACCGGAACCTTCATAGCGCTGGACTGGCTGATGCAGCACATCCGGGACCATGAATTTGTCGATATCTTGGGGCT CGTGAGTGAGTTGCGCTCTCACAGGATGTCTATGGTGCAGACAGAGGAGCAGTTTGTGTTCATCCATCAGTGCG CGCAGTTGATGTGGAAGAAGAAAACCCCGTGCAGCTT

$>$ PTPRO.fish

AGTGATTTCGAGGCCTACCTCAAAGACATGGGCAAAGACTCAGCCTACAAGTTCTCCCTGCAGTTCGAGGAGCT GAAGAGTGTAGGTCTGGATTTGTCCCATGAAGCCGCTGATCTGCCCATCAACAGACCCAAGAACCGCTACACCA ACATCCTGCCCTATGACTTCAGTCGAGTGAAGCTGATCTCTCTGCACAATGATGAAGGCTCTGATTACATCAAT GCTAACTATATTCCCGGCTATAACTCTCCACGCGAGTACATCGCCACGCAGGGCCCACTGCCCGACACCAGGAA CGACTTCTGGAAGATGGTGCTGCAGCAGAAAGTGCACATCATCGTGATGCTAACACAGTGCAACGAGCGCCGCA GGGTGAAGTGTGACCACTACTGGCCGTTCAGTGATGAGCCGGTGGCGTATGGAGAGATCAGTGTGGAGATGCTG GCGGAGACAGACTCGCCCGAGTGGACCATCAGGAGCTTCAGACTCGCATACGCTGATGAGACTCAGGACGTCCT GCACTTTAACTACACCTCGTGGCCGGATCACGGAGTGCCGACGGTGAACGCCATCGAGAGCATCCTACAGTTCG TGCAGATCGTCCGGCAGCAGGTCAACAGGAGCAAAGGACCCATCGTAGTGCACTGCAGTGCTGGCGTGGGCCGC ACCGGCACCTTCATCTCTCTGGACCGGCTGATGCAGCACATTCAGGAGCACGAGTATGTGGACGTGCTGGGGCT GGTGTCCGACATGCGCTCGCACCGACTGTCCATGGTGCAGACTGAGGAGCAGTACGTGTTCATCCATCAGTGTG TTCTGCTGATGTGGAAGAAGAAG

$>$ PTPRH.human

GAAGACTTCGCTGACCACGTCAGGAAGAATGAGAGGGACAGCAACTGTGGTTTTGCAGACAAGTACCAGCAACT CTCCCTGGTGGGCCACAGCCAGTCTCAGATGGTGGCTTCGGCTTCAGAGAACAACGCCAAGAACCGCTACAGAA ATGTGCTGCCCTATGACTGGTCCCGGGTGCCCCTGAAGCCCATCCATGAGGAGCCAGGCTCTGACTACATCAAT GCCAGCTTCATGCCCGGTCTCTGGAGCCCCCAGGAGTTCATTGCAACCCAGGGTCCCCTGCCACAGACAGTGGG TGACTTCTGGCGCCTGGTGTGGGAACAGCAGAGCCACACCCTGGTCATGCTGACCAACTGCATGGAGGCCGGCC GGGTGAAGTGTGAGCATTACTGGCCTCTGGACTCGCAGCCCTGCACCCATGGGCACCTGCGGGTAACCCTGGTA GGTGAGGAAGTGATGGAGAACTGGACGGTGCGGGAACTGCTGCTCCTCCAGGTGGAGGAGCAGAAGACACTGTC TGTGCGCCAATTCCACTACCAGGCCTGGCCGGATCACGGCGTTCCCTCCTCCCCAGACACCTTGCTGGCTTTCT GGAGGATGCTTCGGCAGTGGCTGGATCAGACCATGGAGGGAGGCCCACCCATTGTGCACTGCAGTGCTGGCGTG GGTCGCACAGGAACCCTCATTGCCCTGGACGTCCTGCTCCGGCAGCTGCAGTCCGAGGGTCTCCTTGGGCCCTT CAGCTTTGTAAGGAAGATGAGAGAGAGTCGGCCGTTGATGGTGCAGACTGAGGCTCAGTACGTATTCCTGCATC AGTGCATCCTGCGGTTCCTCCAACAGTCA

$>$ PTPRH.mouse

AAAGACTTTGCTGACCATGTCAGGGAGAATGAGAAGGACAGCAACTGTGGATTTGCTGAGGAGTATCAGCAACT GGCTCTGGAGGGCCAAGGCCAGTCTCAAATAACGGCATCGGCTCTAGAGAACAGATCCAAGAACCGCTACAGGA ATGTACTGCCCTATGACTGGTCCCGGGTTCCCCTGCAGCCCCTCCAAGAGGAACCAGGCTCTGACTATATCAAC 
GCCAGCTTTATGCCTGGTCTCTGGAGCCCCAAGGAGTTCATTGCAACCCAGGGTCCCCTGCCTAACACTGTGGG TGATTTTTGGAGAATGGTCTGGGAACAGCAGAGCCACACCCTGGTTATGCTGACCAACTGCATGGAGTCTGGAC GGGTGAAGTGTGAGCACTACTGGCCCTTGGATGCTCAGCCTTGCATTCATGGGCAGCTGCAGGTAATGTTGATT AGTGAGGAGGCGTCAGAAAACTGGACAGTGAGGCATCTTCAACTCTTCCATATGAAAGAACAGCAGACTCTCTC GTTGCGCCAATTCCACTACCTGGCCTGGCCAGACCATGGAGTTCCTTACTCCCCAGACCCCCTGCTGGCTTTCC GAAAGATGCTTCGGCAATGGATGGATCAGACCACGGATGGAGGTCCACCCATTGTGCATTGCAGTGCTGGTGTG GGCCGGACGGGCACCCTAATTGCACTGGATGTCCTGCTGCGGCAGCTGGAGTGTGAGGGGCTGGTGGGTCCCTT CAGCTTTGTGAAGAAGATGAGAGAGAGCCGGCCACTGATGGTGCAGACAGAGGCCCAGTATGTGTTCCTCCACC AGTGCATCTTGAAATCCCTCCAGAAGCCA

$>$ PTPRH.frog

TCAGCGTTTCCAGATTATTACCAGAGACAGCACGCGGATAGTGACTTTGGATTTGCAGAGGAGTATCAGCAACT CAGCAATGTAGGCATTAACCAGTCTAAACTGGCAGCAGAACTCTCAGAAAACAGATCCAAGAACAGATTCACCA ATGTCTTGCCTTATGACCATTCCAGAGTCAGACTCAACCGTATTGATGCGGATGAGACCTCTGATTATATCAAT GCCAACTACATGCCTGGCTACAACTCTTCTAAGGAATTCATAGCTTCCCAAGGCCCACTGCCCAACACAAGTGC AGACTTCTGGAGGATGATTTGGGAGAATCAAGTCAGTACCATTGTAATGCTGACCAACTGCATGGAGAATGGCA GGGTGAAATGTGAACATTATTGGCCCTTGGATTACACCCCATGCACATACGGAGACATCACCGTGACTGTAACC TCCGAAATGATCCTTCCTGATTGGACAGTACGGGATTTTACCCTTAAACATGCCAAGCAGCAAGGCAATAAGCA TGCTCGTCATTTTCACTTTACGGTCTGGCCCGACCACGGTGTTCCAGAAAACACAACAACCATTGTCGAGTTCC GGAACCTTGTTCGAGAATACATGGACCTGAAGAGGAGCAGCGGACCCACGGTTGTTCACTGCAGTGCTGGTGTT GGGAGGACAGGGACACTGATTGCCCTGGATTACCTGATCCAGAAGATGGAGAAGGAGCAGCGTATTGGAATATA TAGCTTTGTTCAGAAGATGAGGCAGAACCGTCCCTTAATGGTGCAGACAGAGAGTCAGTACGTATTTCTGAATA AGTGTATGCTGGATCTAATCCAGAATCCTCCC

>PTPRH.fish

GATAAATTTCCTGAACATTTCCGAAACATGAGTCGTGATGATAACAGAGCTTTCAGTGCTGAATATGATGACTT GAGTTCAGTGGGTGTAGAACA GTCAAAGGTTGCGGCTCTTCTCCCTGAAAACAAGGATAAAAATCGATTCTCCA ATGTTTTACCATATGACACCTCTAGAGTGCATCTTACCATAAATAAAGCGGGTGATTCTGATTACATCAATGCT AACTACATGCCTGGATATGGAAATGCAAGCAGAGAGTACATAGCTGCTCAAGGTCCACTGCCATCCACTGTCAA TGACTTCTGGAGAATGATCTGGGAGAAAAAGTCATCGACTATTGTCATGGTTACCAACTGTACTGAGGGTGGAA GGGTCAAGTGTGAACAGTACTGGCCACTGGACTACACACCGTGTCTTTATGAAAACCTGCTTGTCACAGTGAAA TCAGAGAACAAATCCCAAAGTTGGACTTTGCGAGAATTTAATGTCAAAAATAAAATGACCTCTGAGACGCGAAC AGTGCGTCACTTCCACTTCACAGCATGGCCGGATCATGGTGTTCCCCGGGGCACAGAAGAGCTGATCCAATTCA GAGACCTTATCCGTCAGCACATAGAGAGCCACTTCTCTACAGGACCAACAGTAGTGCACTGCAGTGCTGGAGTG GGCCGGACAGGGACGCTGATCGCGCTGGACGTCCTGCTGCAGCAGCTGAACAGAGAGAAGGCAGTGGGTGTAGC AGCATTCGTCCAGGAAATGAGACTCAACCGGCCACTTATGGTGCAAACGGAGTCTCAGTATGTGTTCCTGCACC AGTGCATCCTGGACTCTTTGCAAACCAAG

$>$ Ciona. 1

GCTGAGTTTCTTGACTTACTTAAAGTCATGAAAGCAGATTCTGATTTTAAATTCTCAGAAGAGTATGAGGAATT CAAGACCGTTGGGCGAGACCAGGCAACAGTTGCTGCTCTGTTGCCCGAAAATCGTGGCAAGAACCGTTACACCA ACATTCTGCCATATGATGCAACTCGTGTAAAGTTATCTGCAATTGACGACGAACAAGGAACGGATTACATCAAT GCTAACTTTATCCCGGGCAACAACAATCGTCAGCGCGAGTACATCGCCACTCAAGGTCCTCTTCCAGGAACAAA GGAAGATTTTTGGAGGATGGTTTGGGAGCAAAACTCGAGGAACATTGTGATGGTGACACAGACTGTGGAGAGAG GCAAGATAAAGTGCGACCACTACTGGCCATTCGACAACGAACCAATCACAGTGGCTGATTACACGCTACAGATG ACATCAGAATCCATTCTTCCTGAGTGGACGATCCGTGAATTCAAGATCACACACGGGTCTGACACTCGTAGGAT CCGACAGTTCCATTACACAGTATGGCCCGATCATGGTGTGCCAGACACCGCTGAAACACTCGTTAAGTTCATTC GTTACGTTCGTCGAACTATTGACAGGGAAGCGAAACATAGCGGACCAACCGTTGTTCACTGCAGTGCTGGAGTT GGTCGTACAGGAACATTTATCGCAATGGATCGACTTCTTCAACATCTTCCCGACAATAATTACGTCGATATCTT TGGGATCGTTCATCAAATGCGAATTCATCGTGTGTTTATGGTGCAGACAGAGAGCCAGTATATCCTCATCCACC AGATGGTTCAAGATATTCTCAACCGAGTT

$>$ AcornWorm. 1

GCTGAATATCATGGATATTTCCAAATTATGAGTGCAGACATGGAGTTCCGCTTCTCAGAAGAATATGATGAACT GCGACCAGTTGGGAGAGAACAGTCTTGGGATGCAGCTGAGTTGCCAGAAAATAGAGCTAAAAATAGATACACCA ACATTCTACCATATGATCGTACAAGAGTTAAACTCTCACAGGTGGAAGATGATGAAGCCACTGATTATCTCAAT GCTAACTGGATGCCAGGTTTCAATTCTCCCAGGGAATTCATCGCTGTACAAGGAACACTGCCAGGTACCAAGGA TGATTTTTGGAGAATGATTTGGGAATATAATGTGAGCACCATTGTCATGGTAACACAATGTCAGGAAAGAGGAC GGGTGAAATGTGAGCGTTATTGGCCAACTGATGATAACCCAGTTTACTATGGTGATGTGTTGGTGACAGTCACT CATGAAAATGAACTGACTGATTGGGTTATCAGGGAATTCACAGTGGAAAACGGCAAGTCACTGAGACGAATACG TCACTTTAATTTCACTGCGTGGCCTGATCATGGTGTACCTGAAGAAACAGGTTCTCTGATCAAGTTTGTACGAT CTGTCAGGGCGCAGATTCAGAATGATGGTACACCAACTGTTGTACATTGCAGTGCTGGTGTTGGTAGGACTGGT ACTTTCATTGCACTAGATAGACTTATACAGCATATAAAGGAGTATGACTACGTGGATATATTTGGTATCGCATG 
TGAAATGAGAATGCATAGAGTCTATATGATCCAAACTGAGTCCCAGTACATATTCATTCATCTTTGTGTGGATG ACCTATTAAAAGAGCGA

$>$ Nematode. 3

GCGGATTTCGCTGAACATGTTCGTATGATGTCTGCCGATTCCGATTTCCGTTTTTCGGAAGAATATGAGATTCT GCGAAACGTTGGCTGTGGGCAATCATACACGGCTGCCGAATTGCCCGTAAATAAACCAAAGAACAGATTCACAA ATATTCTCCCATATGACCATTCCCGTGTTCGATTAATGCAAGCGGGTGATCAAGAAGGCGCCGACTATATCAAT GCCAACTTCATGCCGGGTTACTCTTCACGACGAGAATTCATCGCTGCCCAAGGACCTTTACCAACAACCAGAGA CGCCTTTTGGCAGATGGCATGGGAACAATACTGCCCGGCAATCATTGCGTTAACAAAGTGCGTGGAAAAAGGTC GAGATAAATGTCATCAGTATTGGCCAGACAGTGAACACTCAAGCGTCGTCTATTCCGATATCGAGGTAACCTTG TTGAATGAATCGTCCTATGACGATTTCACGATCCGTGAGCTTCGTCTAAAGAAGTTGCACGAATCGACGGCACC TCGGATCATCTGGCACTTCCACTATATGGCTTGGCCAGATTTCGGCGTACCAGATCATCCACAGGGAATTATTC GATTCGCTCTGATGTTCCGCTCGAGACTTCCGCACTCTGCCCTGAACAAACCGACCATTGTCCACTGCAGTGCT GGAGTCGGAAGGTCTGGTACATTCATAGCGATAGATCGCCTTCTTCAAACGATACAAGCTGATCGACCTATAGA TGTCTTTGGAATTGTGCACGAAATGCGATATGAGCGATGTCATATGGTCCAAAATGAACAACAATACATCTTCA TCCATCATTGCATTCTTCATGCCATCGAGTCTATC

$>$ Nematode. 1

GAAGACTTCGCCGAGCACGTGCGACTGATGTCAGCAGATTCTGATTTCCGATTCTCTGAGGAATACGATATGAT GCGAAACGTGGGTGTTGGACAATCAGTCGCCGCTTCAGAATTGACTGTAAATCGACCGAAGAATCGTTTCACCA ACATTCCTGCCTATGATCATTCCCGTGTGAAGCTGTCAAATCCGAATAATATCGAAGGAGGCGACTACATCAAT GCCAATTATGTTCCCGGATTCTCGTCTCGTCGAGAGTTCATTGCCGCCCAAGGTCCTTTACCATCGACACGTGA TCACTTCTGGCAGATGACGTGGGAGCAGCAGTGTCCAGCGATCATTGCATTGACGAAGTGTGTGGAAAAGGGAC GAGATAAGTGTCATCAGTACTGGCCGGATCATGAGAATGTGCCTGTTTTGTATGGAGATATTGAGGTAACGATA ATGGCTGAAAAAGAGTACGAAGAGTTTGTGATCCGAGATATTCGATTGGAAAAAGTGGGTCCAGAAGGTCGAGC GACCCGATACGTTCGTCATTGGCATTACATGGCGTGGCCAGATTTTGGAGCACCAGCCCATCCGAACGGAATCA TTCAATTCTCTCGAATGTTCCGACATCAGCTACCACATTCACCTCACAACGAACCAACGATAGTACATTGCAGT GCTGGAGTTGGTCGTTCTGGAACCTTCATCAGCATCGATCGTCTCCTACAAACTAGTTCACTTGGAGAGCCAAT CGATGTATTTGGAACAGTTTGTGAGATGCGATATGAGCGGTGTCAGATGGTGCAGAATGAACAACAATACATAT TTATTCATTATTGTGTTCTTCAAGTCCTTCAAGGATCT

>ciona.2

ACCGACTTTTCGTTGTTGGTGGAAAAATTATCACAAAACAAAAATAAAGGTTTTAGTTCAGAATTCGATGACAT TAGGGGAATACCCTATGCTGGTACTACATCTGTAGCAGAAAAAGCTTGTAATAAAACGAAAAACAGAACGGCTA AGCTAGTACCTTTTGATCATTGTAGAGTCAAGATAGAAGGAATACCTGCTGTGGAAGGTTCAAATTACATCAAT GCTTCCTATGTACCGGGTCCCCATAGTCCCGAACAGTACGTGGCCACCCAAACACCTCTGGATCACACAAAGAA AGATTTCTGGAGGTTAATTTGGGAGACGGGTTCAAATACCATTGTGATGTTATGCAACGTCATGGAGGGAGGAA AGAAGAGATGCAGTGAGTATTGGCCGAAAAAACAAACTGAATATTTTGGAAACTTGGCAATTCAGATGACCAAG CAGGATGTATGCAAGGATTGGATTGTTCGACACTTTTCTGTAACAATGCGGGACAAAGTTCGTCATGTGACGCA GTTTCAATTCATGAATTGGTCAGTAGTGGAACAGGGAAACCCCCTTCCATTGGTGCGATTTATTAAAAGTTATC GGACAACGCGTCACAATGAGTACAAACCAACCATTGTTATGTGCAGCAACGGTAGTGGTCGAAGCGGCGTTTTC ATTGGTTTGGACAAAATCGTTGATACTATGACATCATACATCGACGTGTTTGGGACAGTTGCGTCCCTGAGGAA GTATCGGCCGTTTATGGTTCAAACCCTGACTGAATACATCGTAATGCACCAATGCATCGTGAACTTCATTGATG ATGTC 